\title{
A Statistical Field Approach to Capital Accumulation
}

\author{
Pierre Gosselin* $\quad$ Aïleen Lotz ${ }^{\dagger} \quad$ Marc Wambst ${ }^{\ddagger}$
}

Mai 2019

\begin{abstract}
This paper presents a model of capital accumulation for a large number of heterogenous producerconsumers in an exchange space in which interactions depend on agents' positions. Each agent is described by his production, consumption, stock of capital, as well as the position he occupies in this abstract space.

Each agent produces one differentiated good whose price is fixed by market clearing conditions. Production functions are Cobb-Douglas, and capital stocks follow the standard capital accumulation dynamic equation. Agents consume all goods but have a preference for goods produced by their closest neighbors.

Agents in the exchange space are subject both to attractive and repulsive forces. Exchanges drive agents closer, but beyond a certain level of proximity, agents will tend to crowd out more distant agents.

The present model uses a formalism based on statistical field theory developed earlier by the authors. This approach allows the analytical treatment of economic models with an arbitrary number of agents, while preserving the system's interactions and complexity at the individual level.

Our results show that the dynamics of capital accumulation and agents' position in the exchange space are correlated. Interactions in the exchange space induce several phases of the system.

A first phase appears when attractive forces are limited. In this phase, an initial central position in the exchange space favors capital accumulation in average and leads to a higher level of capital, while agents far from the center will experience a slower accumulation process. A high level of initial capital drives agents towards a central position, i.e. improve the terms of their exchanges: they experience a higher demand and higher prices for their product. As usual, high capital productivity favors capital accumulation, while higher rates of capital depreciation reduce capital stock.

In a second phase, attractive forces are predominant. The previous results remain, but an additional threshold effect appears. Even though no restriction was imposed initially on the system, two types of agents emerge, depending on their initial stock of capital. One type of agents will remain above the capital threshold and occupy and benefit from a central position. The other type will remain below the threshold, will not be able to break it and will remain at the periphery of the exchange space. In this phase, capital distribution is less homogenous than in the first phase.

Key words: Path Integrals, Statistical Field Theory, Phase Transition, Capital Accumulation, Exchange Space, Multi-Agent Model, Interaction Agents.
\end{abstract}

JEL Classification: C02, C60, E00, E1.

*Pierre Gosselin : Institut Fourier, UMR 5582 CNRS-UJF, Université Grenoble I, BP 74, 38402 St Martin d'Hères, France. E-Mail: gosselin@ujf-grenoble.fr

$\dagger$ Aïleen Lotz: Cerca Trova, BP 114, 38001 Grenoble Cedex 1, France. E-mail: a.lotz@erc-cercatrova.eu

¥Marc Wambst : IRMA, UMR 7501 CNRS, Université de Strasbourg, France. E-Mail: wambst@math.unistra.fr 


\section{Introduction}

Field theory applied to economic models is useful to overcome the pitfalls of aggregation. This approach developed in Gosselin, Lotz and Wambst $(2017,2018)$ preserves the microeconomic concepts of standard economic models to describe fully or partly rational agents, while enabling the study of the transition from individual to collective scale given by statistical physics. It provides an analytical treatment of a broad class of economic models with an arbitrary number of agents, while keeping track of the system's interactions and complexity at the individual level.

Field theory describes an environment of an infinite number of interacting agents, from which various phases or equilibria may emerge. It allows to study the agents' behaviors, the way they are influenced by and interact with their environment. Depending on the parameters of the system, the form of the ground state may drastically change the description at the individual level. It is thus possible to compare the features of the macro state of a system and those of the micro level, and their interactions. As such, it may confirm or invalidate some aspects of the representative agent models and shed light on up-to-now discarded micro and macro phenomena.

The present paper applies this field formalism to a model of capital accumulation for a large number of heterogenous producer-consumers in an exchange space in which interactions depend on agents' positions. Each agent is described by his production, consumption, stock of capital, as well as the position he occupies in an abstract space of exchanges. Each agent produces one differentiated good whose price is fixed by market clearing conditions. Production functions are Cobb-Douglas, and capital stocks follow the standard capital accumulation dynamic equation. Agents consume all goods but have a preference for goods produced by their closest neighbors. Thus, demand depends not only on prices, but also on the distance between consumers and producers in the exchange space. The closer the agents, the higher their propensity to exchange, the higher the demand. Moreover, the position of each agent is itself dynamic. Agents in the exchange space are subject both to aggregation and repulsion forces. Exchanges drive agents closer, but beyond a certain level of proximity, closest agents will crowd out more distant agents: an increased proximity weakens more distant exchanges.

The dynamic exchange space presented in this model allows to study the production, exchanges, market shares and capital accumulation within a large group of agents. What are the patterns of accumulation across agents? Is there a threshold effect leading some producers to accumulate at the expense of the others? Is there a phase in which a better wealth distribution could be reached? All these questions can be addressed within our formalism.

Translating standard economic models into a statistical field model is a two-step process. In a first step, the usual model of optimizing agents is replaced by a probabilistic description of the system. In such a setting, individual optimization problems are discarded. Each agent is described by a time-dependent probability distribution centered around this agent's classical optimization path. In a second step, the individual agents' description is replaced by a more compact model of field theory that replicates the properties of the system when $N$, the number of agents, is large (Gosselin, Lotz and Wambst 2017, 2018, and Kleinert 1989). This modeling, although approximate, is compact enough to allow an analytical treatment of the system.

In our model, this formalism yields the probabilistic dynamics of individual agents through the computation of so-called transitions functions. Given an initial stock of capital and an initial position in the exchange space, each individual stochastic path can be found, and depends on parameters such as strength in exchange interactions, rate of capital depreciation and uncertainty in economic variables. We show that, depending on the interaction forces in the exchange space, two phases of the system appear. One corresponds to a society in which the repulsion force is a large enough to compensate the attractive force resulting from the exchange. We show that in that phase capital accumulation and mobility in the exchange space depend highly on the initial capital stock. However, this phase presents a certain stability and allows, up to a point, some capital accumulation for most agents. The second phase appears for an attractive force larger than the repulsive one.

The first section details the literature review. Section two describes a classical model of capital accumulation with $N$ economic agents, translates it into a probabilistic framework and presents its associated field formulation. In section three, we solve the dynamics of the system and present its various phases. Section four interprets the results and section five concludes. 


\section{Literature review}

A large branch of the recent economic literature has been devoted to address the notion of representative agent. Complex systems, Networks, Agent Based Systems or Econophysics are among the various paths that have been explored to remedy its pitfalls.

By several aspects, our approach is related to the Multi-Agents System economic literature, notably Agent Based Models (see Gaffard and Napoletano 2012) and Economic Networks (Jackson 2010). Both rely on numerical simulation of Multi-Agents System but are often concerned with different types of model. Agent Based Models deal with general macroeconomics models, whereas Network Models rather deal with lower scale models, such as Contract Theory, Behavior Diffusion, Information Sharing or Learning. In both type of settings, agents are typically defined by, and follow, various set of rules. These rules allow for equilibria and dynamics that would otherwise remain inaccessible to the representative agent setup.

The Agent-Based approach is similar to ours in that it does not seek to aggregate all agents but considers the interacting system in itself. It is however highly numerical, model-dependent, and relies on microeconomic relations, such as ad-hoc reaction functions, that may be too simplistic. On the contrary, Statistical Field Theory accounts for the impact of scale changes. Macroeconomic patterns do not emerge from the sole dynamics of a large set of agents but are grounded on particular behaviors and interactions structures. Describing these structures in terms of Field Theory allows to study the emergence of a phase at the macro scale, and in turn its impact at the individual level.

Econophysics is closer to our approach (for a review, see Chakraborti, Muni Toke, Patriarca and Abergel 2011a, b and references therein). It often considers the set of agents as a statistical system. Moreover, Kleinert (2009) has already used path integrals to model the stock prices' dynamics. However, Econophysics does not apply the full potentiality of Field Theory to economic systems. It focuses on empirical laws, but the lack of micro-foundations casts some doubts on the robustness of these observed empirical laws, that are prone, like ad-hoc macroeconomics, to the Lucas critique (see Lucas 1976). Our approach, in contrast, keeps track of usual microeconomics concepts such as utility functions, expectations, forward looking behaviors. It includes these behaviors in the analytical treatment of Multi-Agents Systems by translating the main characteristics of a system of optimizing agents in terms of a statistical system.

Capital accumulation has been considered in several ways since the Solow growth model (Solow 1957) and its subsequent developments (see Barro 1995 for an account). The closest approaches to this paper stems from Economic Geography (Krugman 1999) and introduce several types of producers in a differentiated geographical environment, "core" and "periphery". This environment impacts the production of partly differentiated goods, such as agricultural and manufactured goods (see Fujita and Thisse 2013 for a review). There is a notion of space in these models, but the position of agents is static, whereas in our approach, exchange position is dynamic and interacts with capital accumulation. Moreover, although conditions for takeoff and convergence are studied for regional industrialization or multi countries growth models (Aghion and Durlauf 2005), it is the fixed geographical parameters that determine the environment, notably transportation costs and shares of immobile workers. The present approach, on the contrary, considers an evolutive environment, that may be endogenized to interact with capital accumulation.

Also related to our purpose, a recent combination of evolutionary theory, complex systems and agentbased model (Gintis 2007) has allowed a detailed study of a large number of producers' capital dynamics (Dosi and Nelson 2010; Dosi, Fagiolo and Roventini 2010; Dosi et al. 2015; Dawid et al. 2011, 2014), (Ciarli et al. 2010; Mandel et al. 2010; Wolf et al. 2013). More refined models investigate how several production sectors interact and compete, using neighbors' output as inputs (Mandel et al. 2016; Mandel 2012). Competition and capital accumulation are driven by random changes in technology, and producers progressively adapt their production via imitation, replacing parts of their inputs with newer, more efficient technologies. In these models, the firms' interactions dynamics are simulated numerically and track the persistence of heterogenous production sectors and the computation of emergent macro quantities such as total output, wages or unemployment. This focus on the evolution of independent sectors is close to our purpose. However, we do not use numerical methods and consider an exchange space determining exchanges between agents. Moreover, we do not focus on the evolution on technology, even if it could be included, as in Gosselin, Lotz and Wambst (2018), but rather study the impact of the exchange dynamics on agents' capital accumulation. 


\section{Description of the model}

This section describes a standard model of capital accumulation for a large number of agents. The usual capital dynamics and production function are maintained, but here agents interact dynamically through an exchange space.

\subsection{Setup}

There are $N$ consumer-producer agents. Each agent is differentiated by his position on an exchange space, denoted $X_{i}(t) \in[-1,1]$. This exchange position is a dynamic variable that interacts with the other variables of the model. It can be seen as a geographic space, but also as an abstract exchange space, in which the central position $X=0$ ensures higher exchanges.

Each agent produces a single differentiated good. Production functions are Cobb-Douglas. Each agent $i$ individual capital stock is denoted $K_{i}(t)$. It is a fully liquid capital whose price is set to one. Since agents are individual producers, labor can be discarded, and we further assume a constant technology factor $A$. So that the individual production function is of the form $A K_{i}^{\alpha}(t)$. The price of each good $P_{i}(t)$ is determined by market-clearing condition. Ultimately, the agent's income is the product of his production and his price:

$$
Y_{i}(t)=P_{i}(t) A K_{i}^{\alpha}(t)
$$

Each agent consumes all the goods produced. We denote $C_{i}^{(j)}(t)$ the consumption of good $j$ by agent $i$ at time $t$. Three factors determine the agents' consumption of each good. First, the quantity of each good that an agent consumes is proportional to his income, with a proportionality factor to consume $\kappa(0<\kappa<1)$.

Besides, the consumption of each good will also be a decreasing function of the good's relative price, and of the distance between its producer and consumer. We detail these two last conditions below.

Agents' consumption depends on the relative price level of goods: we assume that agent's $i$ consumption of good $j$ is a decreasing function $g\left(R_{i, j}(t)\right)$ with $R_{i, j}(t)=P_{j}(t) / \bar{P}_{i}(t)$. The ratio $R_{i, j}(t)$ is the relative price of good $j$ with respect to a general subjective price level for agent $i$. We chose a dependency of the type $g\left(R_{i, j}\right) \sim\left(R_{i, j}\right)^{-(1+\gamma)}$. We define $\bar{P}_{i}(t)=P_{K}^{1-\varepsilon}\left(\hat{P}_{i}(t)\right)^{\varepsilon}$, where $0<\varepsilon<1$. Thus, the price level $\bar{P}_{i}(t)$ is a combination of the general price of capital $P_{K}$ and a subjective location-dependent consumption price index $\hat{P}_{i}(t)$. Since the price of capital is set to $1, \bar{P}_{i}(t)=\left(\hat{P}_{i}(t)\right)^{\varepsilon}$, and the ratio $R_{i, j}(t)$ rewrites $P_{j}(t) /\left(\hat{P}_{i}(t)\right)^{\varepsilon}$. The index $\hat{P}_{i}(t)$ is a weighted average of prices, where each weight is a function of the distance between the consumer and producer. Thus, it depends on the agent's exchange position at each moment of time, and writes:

$$
\hat{P}_{i}(t)=\frac{1}{d} \sum_{j} P_{j}(t) \exp \left(-d_{i j}(t) / d\right)
$$

with:

$$
d_{i j}(t)=\left|X_{i}(t)-X_{j}(t)\right|
$$

and $d$ a constant parameter. The factor $\frac{1}{d}$ inside the exponential models the fact that agents interact in average on an interval of length in the exchange space. The factor $\frac{1}{d}$ outside the exponential acts as a normalization factor.

Agent's consumption also depends on the distance between consumer and producers. We assume consumption to be an exponentially decreasing function of the distance between consumer $i$ and producer $j,\left|X_{i}(t)-X_{j}(t)\right|$. Recall that $X_{i}(t)$ and $X_{j}(t) \in[-1,1]$. In analogy with the price level, we chose a dependency of the form $\exp \left(-d_{i j}(t) / d\right) / d$. This exponentially decreasing factor has only a relative value. It may reflect the agents' connections: the position in the exchange space only indicates the exchanges agents establish within the exchange space. It could also account for transportation costs in a geographic 
interpretation. It also implies that an agent at the center of the exchange space will face a higher demand for his good.

Under the previous hypotheses, consumption of good $j$ by agent $i$ writes:

$$
C_{i}^{(j)}(t)=\frac{\kappa Y_{i}(t)}{\left(R_{i, j}(t)\right)^{1+\gamma}} \frac{\exp \left(-d_{i j}(t) / d\right)}{d}
$$

For the sake of simplicity we normalize $(1+\gamma) \varepsilon=1$ in the sequel, but this factor could be reintroduced without impairing the results.

The consumption function of good $j$ by agent $i$ rewrites:

$$
C_{i}^{(j)}(t)=\frac{\frac{\kappa}{d} Y_{i}(t)}{P_{j}^{1+\gamma}(t) / \hat{P}_{i}(t)} \exp \left(-\frac{d_{i j}(t)}{d}\right)
$$

Remark that agent $i$ propensity to consume depends on his relative price index, and thus is location. More precisely, the dependence in $X_{i}(t)$ of this propensity follows the pattern:

$$
\frac{1}{d^{2}} \sum_{j} \exp \left(-d_{i j}(t)\right)\left(\sum_{k} \exp \left(-\frac{d_{i k}(t)}{d}\right)\right)
$$

Replacing the summation by an integral, equation (44) is proportional to $\left(1-\cosh \left(\frac{\left|X_{i}(t)\right|}{d}\right) \exp \left(-\frac{1}{d}\right)\right)^{2}$. All things equal, the agent's propensity to consume is maximal for $X_{i}(t)=0$, minimal for $X_{i}(t)=-1$ and $X_{i}(t)=1$. The individual marginal propensity to consume is thus higher in the center of the exchange space than at the periphery, reflecting the fact that exchanges are a decreasing function of the distance between agents.

The above assumptions reflect the fact that exchanges are more frequent at the center of the exchange space. Actually, this space can be seen as a scale of exchanges, in which the position of the agent measures the intensity of his exchanges. Moves towards the center or the periphery depict respectively an improvement or a deterioration of his terms of exchanges. We define the terms of exchanges of a producer as his ability to sell his production at a given price within this exchange space - competitors and consumers, depending on their capital, revenue, distance from the producer, etc.

To conclude this section, note that the proportionality factor $\kappa$ should be determined by optimization of an intertemporal utility function under the constraint of future flow of expected profits. However, assuming some autonomous consumption proportional to the agent's revenue, $\kappa$ is constant in first approximation. Agents consume an average "minimal" necessary level of goods, given their position in the exchange space, and reinvest the full amount of their remaining income. In our setting, the creation of trade relations compensates the income loss due to a high production. This favors capital accumulation and ultimately, through a better position in the exchange space, increases the producer price.

\subsection{Classical description of the model}

In this setting, each good's price at each point in time is determined by market clearing conditions. The global demand for good $i$ at time t by all agents $j, \sum_{j} C_{j}^{(i)}(t)$ matches the production of good $i$ :

$$
\sum_{j} C_{j}^{(i)}(t)=A K_{i}^{\alpha}(t)
$$

Using (3) and (1), this equation can be rewritten as:

$$
\frac{\kappa}{d^{2}} \sum_{j, k} P_{j}(t) K_{j}^{\alpha}(t) P_{k}(t) \exp \left(-\frac{d_{i j}(t)+d_{k j}(t)}{d}\right)=P_{i}^{1+\gamma}(t) K_{i}^{\alpha}(t)
$$


Capital accumulation dynamics follows a standard pattern. Capital depreciates at rate $\delta$, and capital accumulation is subject to a shock $\epsilon_{i}(t)$. We further assume that revenue saved is entirely reinvested in capital at a price 1 . In such a setting, the capital dynamic equation is:

$$
K_{i}(t+1)=(1-\delta) K_{i}(t)+Y_{i}(t)-\sum_{j} P_{j}(t) C_{i}^{(j)}(t)+\epsilon_{i}(t)
$$

Using (3), we find:

$$
K_{i}(t+1) \simeq(1-\delta) K_{i}(t)+Y_{i}(t)-\frac{\kappa}{d^{2}} Y_{i}(t) \sum_{j, k} \frac{P_{k}(t)}{P_{j}^{\gamma}(t)} \exp \left(-\frac{d_{i j}(t)+d_{i k}(t)}{d}\right)+\epsilon_{i}(t)
$$

$\epsilon_{i}(t), \epsilon_{i}^{(1)}(t), \epsilon_{i}^{(2)}(t)$ variance $\sigma^{2}$

\subsection{Probabilistic Description}

Three dynamic variables $K_{i}, P_{i}$ and $X_{i}$ describe our model. Each agent dynamics is described by a path within the space defined by these three variables: from an initial point in $K_{i}, P_{i}$ and $X_{i}$, the agent reaches a final point in this same space. Classically, up to some fluctuations, an optimal path does exist for each agent.

For a large number of agents however, because of fluctuations, all possible paths may exist with varying probabilities. So that, for a large number of agents, we must take into account the set - or space - of all paths, and associate to this set a probability density centered around the classical optimal path1 1 . This description is a good approximation of standard descriptions and translates the fact that each agent experiments some idiosyncratic shocks.

Once this probability density computed for an individual agent, the probability density for the set of all agents is merely the product of the individual probability densities. This probability density for a configuration of $N$ arbitrary individual paths is called the statistical weight of a state of the system. By construction, it is centered around one - or several in case of multiple equilibria - configuration of paths that represent the classical equilibrium. Its shape need not be known, since we are working directly with statistical weight.

In this section, we provide a probabilistic description of the model. To do so we build a statistical weight for each equation of the model. Their product will be the probability description of the system. The two first variables, $K$ and $P$, are standard economic variables, and their weight will be derived from the equations of the model, as proposed in Gosselin, Lotz, Wambst (2018). The last variable $X$ is not a strictly standard economic variable. We will depart from our methodology and ascribe to its dynamics an ad hoc form.

\subsubsection{Probabilistic description for capital dynamics}

We associate a probability to the dynamic accumulation of capital, centered around the average classical capital dynamics solution, for each period of time $t$. To do so, note that (7) implies that the quantity:

$$
K_{i}(t+1)-(1-\delta) K_{i}(t)+Y_{i}(t)-\frac{\kappa}{d^{2}} Y_{i}(t) \sum_{j, k} \frac{P_{k}(t)}{P_{j}^{\gamma}(t)} \exp \left(-\frac{d_{i j}(t)+d_{i k}(t)}{d}\right)
$$

is a gaussian random variable $\epsilon_{i}(t)$ of variance $\sigma^{2}$. In our context, equation (7) is replaced by the probability density for $K_{i}(t)$ :

$$
\exp \left(-\frac{1}{2 \sigma^{2}}\left(\dot{K}_{i}(t)+\delta K_{i}(t)-A P_{i}(t) K_{i}^{\alpha}(t)\left(1-\frac{\kappa}{d^{2}} \sum_{j, k} \frac{P_{k}(t) \exp \left(-\frac{d_{i j}(t)+d_{i k}(t)}{d}\right)}{P_{j}^{\gamma}(t)}\right)\right)^{2}\right)
$$

\footnotetext{
${ }^{1}$ We use the word probability density rather than probability, since due to the infinite number of possible paths, each individual path has a null probability to exist, just like a gaussian defining the size of a population.
} 
To account for the dynamics over the whole timespan, we sum over $t$ in the exponential. This associates a density probability for a path of capital accumulation over the whole timespan. This will account for stochastic paths $K_{i}(t)$ that satisfy in average the classical dynamic accumulation equation.

Ultimately, to associate a statistical weight to the set of paths of capital accumulation for all agents, we sum over $i$ and $t$ in the exponential of (9). The statistical weight associated to the capital accumulation of the set of agents is thus:

$$
\exp \left(-\frac{1}{2 \sigma^{2}} \sum_{i} \int\left(\dot{K}_{i}(t)+\delta K_{i}(t)-A P_{i}(t) K_{i}^{\alpha}(t)\left(1-\frac{\kappa}{d^{2}} \sum_{j, k} \frac{P_{k}(t) \exp \left(-\frac{d_{i j}(t)+d_{i k}(t)}{d}\right)}{P_{j}^{\gamma}(t)}\right)\right)^{2} d t\right)
$$

\subsubsection{Probabilistic description for market clearing condition}

The dynamics for $P_{i}(t)$ can be replaced, as for capital dynamics, by a statistical weight derived from for the market clearing condition. We assume that market clearing holds in average for a large number of agents, but that fluctuations appear for individual agents. Thus (5) only holds up to some random noise, and must be replaced by a probability for each agent $i$ to deviate from (5).

$$
\exp \left(-\frac{1}{2 \sigma_{1}^{2}}\left(P_{i}^{1+\gamma}(t) K_{i}^{\alpha}(t)-\kappa \sum_{j, k} P_{j}(t) K_{j}^{\alpha}(t) P_{k}(t) \exp \left(-\frac{d_{i j}(t)+d_{k j}(t)}{d}\right)\right)^{2}\right)
$$

with $\sigma_{1}^{2}$ normalized to $\frac{\sigma^{2}}{A^{2}}$ in the sequel where $\bar{A}^{2}$ is a constant. We consider market clearing as a more binding condition than capital accumulation, so that $\bar{A}^{2}>>1$. As for the capital, we associate a statistical weight for the system to the market clearing condition:

$$
\exp \left(-\frac{\bar{A}^{2}}{2 \sigma^{2}} \sum_{i} \int\left(P_{i}^{1+\gamma}(t) K_{i}^{\alpha}(t)-\kappa \sum_{j, k} P_{j}(t) K_{j}^{\alpha}(t) P_{k}(t) \exp \left(-\frac{d_{i j}(t)+d_{k j}(t)}{d}\right)\right)^{2} d t\right)
$$

\subsubsection{Probabilistic description of the exchange space dynamics}

The exchange position $X$ does not correspond to a usual economic variable. We could postulate its dynamic equations, along with its interactions with the other economic variables, and from there, deduce its probabilistic description for an arbitrary number of agents. However choosing an ad hoc form is equivalent, simpler and faster.

We postulate three types of forces governing agents' dynamics within the exchange space and directly write the associated statistical weights.

A first force applies to all agents whatever their position and attracts them towards the center of the exchange space. Without this force the system would not exist or would tend to disintegrate. This force could be a political or social structure assuring the cohesion and exchanges of the group. More broadly, it can also represent the set of all factors insuring a minimal level of exchanges for each good. In the following, we will refer to this force as the "cohesion force".

We postulate a second force induced by the exchanges existing between agents. We suppose that exchanging agents create connections that will smooth their further exchanges, and get closer within the exchange space.

Finally, we postulate a third force that counterweight the second force. We suppose that a small group of agents, i.e. close and exchanging, tend to repell potential new entrants. This force can model exclusive connections such as clientelism or various degrees of market openness.

Considering these three assumptions, we chose the following statistical weight for the all set of agents:

$$
\exp \left(-\sum_{i} \int\left(\frac{\left(\dot{X}_{i}(t)\right)^{2}}{\sigma_{X}^{2}}+V_{0}\left(X_{i}(t)\right)+\sum_{j} V_{1}\left(d_{i j}(t)\right)+\sum_{j, k} V_{2}\left(d_{i j}(t), d_{i k}(t), d_{j k}(t)\right)\right) d t\right)
$$


where $\sigma_{X}^{2}$ is a constant parameter measuring the inertia of $X_{i}$. For $\sigma_{X}^{2}<<1$, the variable $X_{i}$ presents a strong inertia, whereas, for $\sigma_{X}^{2}>>1$ the variable $X_{i}$ adjusts freely.

This statistical weight describes a random dynamic for the variables $X_{i}(t)$. The first term $\frac{\left(\dot{X}_{i}(t)\right)^{2}}{\sigma_{X}^{2}}$ represents the inertia of the variable $X_{i}(t)$. The variation of $X_{i}(t)$ over one period, measured by $\dot{X}_{i}(t)$, is in average of order $\sigma_{X}$ : the value of $X_{i}(t)$ cannot be changed instantaneously.

The three other terms represent the forces acting on each individual agent, but also on groups of various size.

The term $V_{1}$ represents an attraction force between agents exchanging at the individual level.

$$
V_{1}\left(d_{i j}(t)\right)=-\frac{\kappa_{1}}{4} \frac{K_{i}(t) K_{j}(t)}{\langle K\rangle_{X_{i}(t)}\langle K\rangle_{X_{j}(t)}} \exp \left(-\chi_{1} d_{i j}(t)\right)
$$

Two exchanging agents have a tendency to get closer in an exchange space. $\chi_{1}$ is a parameter and $\langle K\rangle_{X_{i}(t)}$ and $\langle K\rangle_{X_{j}(t)}$ are the average capital stock of agents at position $X_{i}(t)$ and $X_{j}(t)$. As (14) shows, $V_{1}\left(d_{i j}(t)\right)$ is proportional to $K_{i}(t) K_{j}(t) /\langle K\rangle_{X_{i}(t)}\langle K\rangle_{X_{j}(t)}$ : the attraction force is proportional to agents' exchanges, revenues, and consequently capital stocks.

The term $V_{2}$ describes some repulsive forces that occur in the interaction of small groups.

$$
V_{2}\left(d_{i j}(t), d_{i k}(t), d_{j k}(t)\right)=\frac{\kappa_{2}}{6} \exp \left(-\chi_{2}\left(d_{i j}(t)+d_{i k}(t)+d_{j k}(t)\right)\right)
$$

Here, we have chosen interactions within a group of three agents, but this could be generalized to $k$ agents, with $k<<N$, where $N$ is the total number of agents. The idea behind this force is that when several - more than two - agents interact, the interactions they already have deter additional interaction.

The term $\chi_{2}$ is a parameter, and the last term, $V_{0}$ is chosen to be:

$$
V_{0}\left(X_{i}(t)\right)=\frac{\kappa_{0}}{2 \sigma_{X}^{2}}\left(X_{i}(t)-\left\langle X_{i}(t)\right\rangle\right)^{2}
$$

with $\kappa_{0}<<1$. This describes a "weak" global force that tends to regroup all agents towards the center of the exchange space, a cohesion force.

\subsubsection{Probabilistic description of the system}

Gathering the contributions (10), (12) and (13) leads to the probabilistic weight:

$$
\begin{aligned}
& \exp \left(-\frac{1}{2 \sigma^{2}} \sum_{i} \int d t\left(\dot{K}_{i}(t)+\delta K_{i}(t)\right.\right. \\
& \left.\left.-A P_{i}(t) K_{i}^{\alpha}(t)\left(1-\frac{\kappa}{d^{2}} \sum_{j, k} \frac{P_{k}(t) \exp \left(-\frac{d_{i j}(t)+d_{i k}(t)}{d}\right)}{P_{j}^{\gamma}(t)}\right)\right)^{2}\right) \\
& \times \exp \left(-\frac{\bar{A}^{2}}{2 \sigma^{2}} \sum_{i} \int\left(P_{i}^{1+\gamma}(t) K_{i}^{\alpha}(t)-\kappa \sum_{j, k} P_{j}(t) K_{j}^{\alpha}(t) P_{k}(t) \exp \left(-\frac{d_{i j}(t)+d_{k j}(t)}{d}\right)\right)^{2} d t\right) \\
& \times \exp \left(-\sum_{i} \int\left(\frac{\left(\dot{X}_{i}(t)\right)^{2}}{\sigma_{X}^{2}}+V_{0}\left(X_{i}(t)\right)+\sum_{j} V_{1}\left(d_{i j}(t)\right)+\sum_{j, k} V_{2}\left(d_{i j}(t), d_{i k}(t), d_{j k}(t)\right)\right) d t\right)
\end{aligned}
$$

Appendix 0 rewrites this functional in an alternative form, more suitable to switch to the field theory formalism. 


\subsection{Statistical fields description}

Describing the whole system by a statistical weight that accounts for a large number of stochastic paths has allowed us to introduce the various interactions between agents directly inside the statistical weight.

Formally, this statistical weight could be used to study the evolution of the system. Equation (15) would allow to compute the transition probability from an initial state for $N$ agents to a final state. Yet this approach, possible in some cases, is intractable for a large number of agents: it would imply to keep track of the $N$ agents' probability transitions.

It is however a necessary first step (see Gosselin, Lotz and Wambst 2017, 2018) to turn to the more compact field formalism. This formalism replaces the probabilistic description of $N$ agents (15) by a collective field formalism. Rather than tracking $N$ variables, we define an abstract function, the field, that depends only on one single set of variable, here $K, P, X$.

These variables are no more indexed by $i$, the label of individual agents, because field formalism, rather than considering $N$ copies of the same variables and defining a probability for the set of these $N$ copies, defines a probability density on the space of complex valued functions of the variables $K, P, X$.

These complex valued functions $\Psi(K, P, X)$ replace the set of paths used in the previous paragraph. Field formalism reduces the model described by (15) but preserves the essential information contained in the probabilistic description (15) of the system with $N$ agents. Switching to field formalism is a change of perspective: rather than keeping track of agents $1,2, \ldots, N$, this formalism describes dynamics and interactions as a collective thread of all possible anonymous paths. This thread is the environment that conditions the dynamics of individual agents from one state to another.

To each function $\Psi(K, P, X)$, we associate a statistical weight $\exp (-S(\Psi))$ computing the density of probability associated to the a particular configuration $\Psi(K, P, X)$. The functional $S(\Psi)$ is called the field action. The form of $S(\Psi)$ is directly derived from the probabilistic description of our model (15). Technical details about the derivation of the field action $S(\Psi)$ are given in Gosselin, Lotz and Wambst (2017) and a detailed abstract can be found in Gosselin, Lotz and Wambst (2018). In the following, we briefly recall the main steps and Appendix 1 provides some extensions adapted to our purposes.

\subsubsection{Field theoretic formulation}

The set of variables $\left(K_{i}(t), P_{i}(t), X_{i}(t)\right)_{i=1, \ldots, N}$ is replaced by a function $\Psi(K, P, X, \theta)$, where $K, P, X$ represent all the possible values of capital, price and exchange position for a non labelled agent. The parameter $\theta$ is a counting variable and plays the role of time.

The statistical weight is replaced by a weight for this function $\Psi(K, P, X, \theta)$, and describes the probability of a set of configurations $(K, P, X, \theta)$ for all agents. We will define $Z=(K, P, X, \theta)$ and $D_{i j}=\left|X_{i}-X_{j}\right|$. By convention, we will consider that, unless otherwise mentioned, the sign of integration $\int$ refers to all variables involved. The integrations ranges for $K, P$ are $\mathbb{R}^{+}$and for $X$, the interval $[-1,1]$. The transformation from the probabilistic description (52) to a field theoretic model is described in Gosselin, Lotz and Wambst 2017, 2018, and detailed in Appendix 1. The derivation of the results of this section is given in Appendix 2.

We show that the $(K, P)$ part of the field action is:

$$
\begin{aligned}
S_{1}(\bar{\Psi})= & \int \bar{\Psi}^{\dagger}(Z, \theta)\left(-\sigma^{2} \nabla_{K}^{2}-\sigma_{X}^{2} \nabla_{X}^{2}-\vartheta^{2} \nabla_{\theta}^{2}+\frac{1}{\vartheta^{2}}+\alpha\right) \bar{\Psi}(Z, \theta) \\
& +\int\left(\frac{\left(\delta K-A P K^{\alpha}\left(1-\hat{U}_{1}\right)\right)^{2}}{\sigma^{2}}+\frac{\bar{A}^{2}\left(P^{1+\gamma} K^{\alpha}+U_{2}\right)^{2}}{\sigma^{2}}\right)|\bar{\Psi}(Z, \theta)|^{2}
\end{aligned}
$$

with:

$$
\begin{aligned}
\hat{U}_{1} & =\kappa \int \frac{P_{3} \exp \left(-\left(\frac{D_{12}+D_{13}}{d}\right)\right)}{P_{2}^{\gamma}}\left|\bar{\Psi}\left(Z_{2}, \theta\right)\right|^{2}\left|\bar{\Psi}\left(Z_{3}, \theta\right)\right|^{2} \\
U_{2} & =-\kappa \int P_{2}\left(K_{2}\right)^{\alpha} P_{3} \exp \left(-\left(\frac{D_{12}+D_{23}}{d}\right)\right)\left|\Psi\left(Z_{2}, \theta\right)\right|^{2}\left|\Psi\left(Z_{3}, \theta\right)\right|^{2}
\end{aligned}
$$


In the sequel, we simplify the notation by replacing $\bar{\Psi}$ by $\Psi$, but the change of variable will be accounted for while computing the transition functions of the model.

The $X$ part of the weight yields the field contribution:

$$
\begin{aligned}
S_{2}(\Psi)= & \sigma_{X}^{2} \int|\nabla \Psi(Z, \theta)|^{2}+\int V_{0}(X)|\Psi(Z, \theta)|^{2}+\int V_{1}\left(D_{12}\right)\left|\Psi\left(Z_{1}, \theta\right)\right|^{2}\left|\Psi\left(Z_{2}, \theta\right)\right|^{2} \\
& +\int V_{2}\left(D_{12}, D_{13}, D_{23}\right)\left|\Psi\left(Z_{1}, \theta\right)\right|^{2}\left|\Psi\left(Z_{2}, \theta\right)\right|^{2}\left|\Psi\left(Z_{3}, \theta\right)\right|^{2}
\end{aligned}
$$

where:

$$
\begin{aligned}
V_{0}(X) & =\frac{\kappa_{0}}{2 \sigma_{X}^{2}}(X-\langle X\rangle)^{2} \\
V_{1}(|X-Y|) & =-\frac{\kappa_{1}}{4} \frac{K K^{\prime} \exp \left(-\chi_{1}|X-Y|\right)}{\langle K\rangle^{2}} \\
V_{2}(|X-Y|,|X-Z|,|Y-Z|) & =\frac{\kappa_{2}}{6} \exp \left(-\chi_{2}(|X-Y|+|X-Z|+|Y-Z|)\right)
\end{aligned}
$$

and the full following field action $S(\Psi)=S_{1}(\Psi)+S_{2}(\Psi)$ becomes:

$$
\begin{aligned}
S(\Psi)= & \int \Psi^{\dagger}(Z, \theta)\left(-\sigma^{2} \nabla_{K}^{2}-\sigma_{X}^{2} \nabla_{X}^{2}-\vartheta^{2} \nabla_{\theta}^{2}+\frac{1}{\vartheta^{2}}+\alpha\right) \Psi(Z, \theta) \\
& +\int\left(V_{0}(X)+\frac{\left(\delta K-A P K^{\alpha}\left(1-\hat{U}_{1}\right)\right)^{2}}{\sigma^{2}}+\frac{\bar{A}^{2}\left(P^{1+\gamma} K^{\alpha}+U_{2}\right)^{2}}{\sigma^{2}}\right)|\Psi(Z, \theta)|^{2} \\
& +\int V_{1}\left(D_{12}\right)\left|\Psi\left(Z_{1}, \theta\right)\right|^{2}\left|\Psi\left(Z_{2}, \theta\right)\right|^{2}+\int V_{2}\left(D_{12}, D_{13}, D_{23}\right)\left|\Psi\left(Z_{1}, \theta\right)\right|^{2}\left|\Psi\left(Z_{2}, \theta\right)\right|^{2}\left|\Psi\left(Z_{3}, \theta\right)\right|^{2}
\end{aligned}
$$

\section{Resolution}

Several results about the model can be derived from the field action $S(\Psi)$ and its statistical weight $\exp (-S(\Psi))$ : the phases of the system, that describe the collective background of the system, and the transition functions of the system.

The phases of the system are defined by the field $(\mathrm{s}) \Psi_{0}(Z, \theta)$ that maximize(s) the statistical weight $\exp (-S(\Psi))$, i.e. minimize(s) $S(\Psi)$. The field $\Psi_{0}(Z, \theta)$ is the most likely configuration: it represents some collective background field(s) that condition(s) the dynamics of individual agents.

The existence of a minimum for $S(\Psi)(18)$ depends on the parameters of the system. For some values of the parameters, only the trivial phase $\Psi_{0}(Z, \theta)=0$ exists, and amounts to a system linearized around a static equilibrium. When non-trivial phases $\Psi_{0}(Z, \theta) \neq 0$ exist, they reveal other types of equilibria. The configuration $\Psi_{0}(Z, \theta)$ is the background state in which probability transitions and some average values can be computed. Thus, the form of $\Psi_{0}$ has direct implications on the system's dynamics.

The transition functions, or Green functions, for $k$ agents compute, for a given phase, the probability for $k$ agents to evolve from one initial state - the given values of capital, price and exchange position for each of the $k$ agents - to a final state in a certain time span. Thus, the field formalism recovers the random description of one or any number of agents. We will see below that, in a given phase, the $k$ agent transitions functions can be recovered from the single agent transition probability.

To find the transition functions, we use the fact that $\exp (-S(\Psi))$ represents itself a statistical weight for the system. In fact, the second order expansion of $S(\Psi)$ around $\Psi_{0}(Z, \theta)$ yields a quadratic action that determines directly the Green functions of the system. The derivation of this point can be found in (Gosselin, Lotz, Wambst 2018).

In this section, we study the conditions for the appearance of a non-trivial phase for the system described by (18). We find that, depending on the parameters of the system, two potential phases arise. We then compute for each possible phase an "effective" quadratic action. This is a simplified version of (18) in which the price variable can be replaced and the specificities of a given phase are taken into account. This quadratic action is then used to compute the transition functions in each phase. 


\subsection{Possibility of several phase}

In this paragraph, inspecting the configurations $\Psi_{0}(Z, \theta)$ that minimize the action, we find the conditions of appearance of a non-trivial phase for the system.

In the absence of any dynamics for $X$, since the potential for $K, P$ is positive, the minimal configuration is null. Thus, the possibility of non-trivial configuration depends on the $X$ part of the action (17). Using a first approximation for $S_{2}(\bar{\Psi})$ the minimization of (17) yields:

$$
\begin{aligned}
0= & -\sigma_{X}^{2} \nabla_{X}^{2} \Psi(Z, \theta)+V_{0}(X) \Psi(Z, \theta)+\left(\int V_{1}\left(X-X_{2}\right)\left|\Psi\left(Z_{2}, \theta\right)\right|^{2}\right) \Psi(Z, \theta) \\
& +\left(\int V_{2}\left(X-X_{2}, X-X_{3}, D_{23}\right)\left|\Psi\left(Z_{2}, \theta\right)\right|^{2}\left|\Psi\left(Z_{3}, \theta\right)\right|^{2}\right) \Psi(Z, \theta)
\end{aligned}
$$

and we replace some quantities by their average:

$$
\begin{aligned}
\langle X\rangle & =0 \\
\left|X-X_{i}\right| & \simeq \sqrt{\left\langle\left(X-X_{i}\right)^{2}\right\rangle} \simeq \sqrt{2\left\langle X^{2}\right\rangle} \simeq \sqrt{2} \kappa_{0}^{-\frac{1}{4}} \sigma_{X} \\
V_{1}\left(\left|X-X_{2}\right|\right) & =-\frac{\kappa_{1}}{4} \frac{K K^{\prime} \exp \left(-\chi_{1}\left|X-X_{2}\right|\right)}{\langle K\rangle^{2}} \exp \left(-\chi_{1}\left|X-X_{2}\right|\right) \\
& \simeq-\frac{\kappa_{1}}{4} \frac{K K^{\prime} \exp \left(-\chi_{1}\left|X-X_{2}\right|\right)}{\langle K\rangle^{2}} \exp \left(-\chi_{1} \sqrt{2} \kappa_{0}^{-\frac{1}{4}} \sigma_{X}\right) \equiv-\frac{\bar{\kappa}_{1}}{4}
\end{aligned}
$$

and similarly:

$$
V_{2}\left(X-X_{2}, X-X_{3}, D_{23}\right) \simeq \frac{\kappa_{2}}{6} \exp \left(-\chi_{2} 3 \sqrt{2} \kappa_{0}^{-\frac{1}{4}} \sigma_{X}\right) \equiv \frac{\bar{\kappa}_{2}}{6}
$$

In the sequel, the parameters $\chi_{1}, \chi_{2}$ and $\kappa_{0}$ will be considered relatively small. Since we are only concerned with finding approximate conditions for the existence of a non-trivial phase, we can approximate $\bar{\kappa}_{1} \simeq \kappa_{1}$, $\bar{\kappa}_{2} \simeq \kappa_{2}$. We are thus left with an approximated equation:

$$
\begin{aligned}
& 0=-\sigma_{X}^{2} \nabla_{X}^{2} \Psi(Z, \theta)+\frac{\kappa_{0}}{\sigma_{X}^{2}} X^{2} \Psi(Z, \theta) \\
& +\kappa_{1}\left(\int\left|\Psi\left(Z_{2}, \theta\right)\right|^{2}\right) \Psi(Z, \theta)+\kappa_{2}\left(\int\left|\Psi\left(Z_{2}, \theta\right)\right|^{2}\left|\Psi\left(Z_{3}, \theta\right)\right|^{2}\right) \Psi(Z, \theta)
\end{aligned}
$$

We define $\rho^{2}=\int|\Psi(Z, \theta)|^{2}$, and assume a fundamental of the form $\Psi_{0}(Z, \theta)=\Psi_{0}(K) \Psi_{0}(P) \Psi_{0}(X) \Psi_{0}(\theta)$, that we will justify later. Equation (19) rewrites:

$$
0=-\sigma_{X}^{2} \nabla_{X}^{2} \Psi(Z, \theta)+\frac{\kappa_{0}}{\sigma_{X}^{2}} X^{2} \Psi(Z, \theta)+\kappa_{1} \rho^{2} \Psi(Z, \theta)+\kappa_{2} \rho^{4} \Psi(Z, \theta)
$$

The associated fundamental eigenvalue satisfies:

$$
\frac{1}{2} \kappa_{0}^{\frac{1}{2}}-\kappa_{1} \rho^{2}+\kappa_{2} \rho^{4}=0
$$

Thus for $\kappa_{1}^{2}-2 \kappa_{0}^{\frac{1}{2}} \kappa_{2}<0$ the system has only one phase, the trivial phase $\rho=0$, i.e. $\Psi(Z, \theta)=0$.

On the contrary, for $\kappa_{1}^{2}-2 \kappa_{0}^{\frac{1}{2}} \kappa_{2}>0$, there is a possibility of non-trivial phase with:

$$
\rho \simeq \frac{\kappa_{1}+\sqrt{\kappa_{1}^{2}-2 \kappa_{0}^{\frac{1}{2}} \kappa_{2}}}{2 \kappa_{2}}
$$

The value of $\rho$ will be refined below.

As a consequence, the possibility of a non-trivial phase depends on the relative strength of the repulsive force over the attractive one. A non-trivial phase is possible only for a strong enough repulsive force. 


\subsection{Derivation of the effective action}

For each phase, we derive a simpler form of the "effective action" (18). Due to the binding market clearing condition, the price $P$ in (18) is not a dynamic variable: there are no derivatives in $P$, i.e. $\nabla_{P}$. We will show that this variable can consequently be expressed in terms of $K, X$ and the field. It is this simplification that allows to find a quadratic effective action for a field depending on two variables $K$ and $X$.

\subsubsection{Phase 1: $\rho=0$}

We first inspect the case $\rho=0$. Recall that we assume relatively small individual fluctuations around the market clearing condition, so that $\bar{A}>>A$. We also assume that $\sigma^{2}<1$. The ratio $\frac{A}{A}$ will thus measure the effect of these fluctuations on the system.

As a consequence, in the statistical weight $\exp (-(S(\Psi)))$ with $S(\Psi)$ defined by (18), the potential term:

$$
\int\left(\frac{\left(\delta K-A P K^{\alpha}\left(1-\hat{U}_{1}\right)\right)^{2}}{\sigma^{2}}+\frac{\bar{A}^{2}\left(P^{1+\gamma} K^{\alpha}+U_{2}\right)^{2}}{\sigma^{2}}\right)|\Psi(Z, \theta)|^{2}
$$

is predominant. It implies that $\exp (-(S(\Psi)))$ is peaked around fields minimizing (20). Since the dependence of $\Psi$ in $P$ is static, i.e. no gradient in $P$ appears in $S(\Psi)$, we can consider that the fields minimizing (20) have the form:

$$
\Psi(Z, \theta) \rightarrow \delta(P-F(K, X)) \Psi(K, X, \theta)
$$

In other words, the most likely fields are those of the form (21). Classically, the interpretation is straightforward: due to the market clearing condition, the price is a function $F$ of $(K, X)$.

Inserting (21) into the potential terms (20), the minimization equation of (20) becomes:

$$
\Psi(K, P, X, \theta)\left(-U A K^{\alpha}\left(\delta K-A P K^{\alpha} U\right)+(1+\gamma) P^{\gamma} \bar{A}^{2} K^{\alpha}\left(P^{1+\gamma} K^{\alpha}-V\right)\right) \Psi^{\dagger}(K, P, X, \theta)=0
$$

where $U=1-\hat{U}_{1}$. Appendix 2 shows that inserting (21) in (22) leads to identify:

$$
P=F(K, X)=K^{-\frac{\alpha}{1+\gamma}} f(X)
$$

where $f$ is a trial function that must be identified in (22). We thus arrive to the following equation for $f(X)$ :

$$
\left(f\left(X_{1}\right)\right)^{1+\gamma}=\kappa \int\left(K_{2}\right)^{\frac{\alpha \gamma}{1+\gamma}}\left(K_{3}\right)^{-\frac{\alpha}{1+\gamma}} f\left(X_{3}\right) f\left(X_{2}\right) \exp \left(-\left(\frac{D_{12}+D_{23}}{d}\right)\right)\left|\Psi\left(K_{3}, X_{3}\right)\right|^{2}\left|\Psi\left(K_{2}, X_{2}\right)\right|^{2}
$$

Appendix 2 shows that we obtain in first approximation:

$$
f(X)=D \exp \left(-\frac{|X|}{d(1+\gamma)}\right)
$$

with:

$$
\begin{aligned}
D= & \left(\frac{A}{\delta}\left(1-h \exp \left(-\frac{1}{2 d}\right)\left(1-\frac{\exp \left(-\frac{1}{2 d}\right)}{2}\right)\right)\right)^{\frac{\alpha}{(1-\alpha)(\gamma+1)}} \\
& \times\left(\frac{1+\gamma(1-\alpha)}{(2-\alpha) \bar{\kappa}}\left(\left(\frac{2-\alpha}{1+\gamma(1-\alpha)}\right)^{2}-1\right)\right)^{\frac{1+\gamma(1-\alpha)}{\left(1-\gamma^{2}\right)(1-\alpha)}}
\end{aligned}
$$

Appendix 2 also shows that the average capital stock $\langle K\rangle_{X}$ for agents at position $X$ in the exchange space rewrites:

$$
\langle K\rangle_{X}=\left(\frac{A}{\delta} f(X)\left(1-h \exp \left(-\frac{|X|}{d}\right)\left(1-\frac{\cosh \frac{X}{d}}{\exp \left(\frac{1}{d}\right)}\right)\right)\right)^{\frac{1+\gamma}{1+\gamma(1-\alpha)}}
$$


where $h$ and $\bar{\kappa}$ are defined by:

$$
\begin{aligned}
\bar{\kappa} & =\kappa\left(1-h \frac{A^{2}}{\bar{A}^{2}}\left(1-\exp \left(-\frac{1}{d}\right)\right)\right) \\
h & =\frac{\bar{h}}{\left(1-\frac{A^{2}}{A^{2}} \bar{h}\left(1-\exp \left(-\frac{1}{d}\right)\right)\right)} \\
\bar{h} & =(1+\gamma(1-\alpha))\left(\frac{1+\gamma(1-\alpha)}{2(2-\alpha)}\left(\left(\frac{2-\alpha}{1+\gamma(1-\alpha)}\right)^{2}-1\right)\right)\left(\frac{3}{2}-\frac{e^{-\frac{1}{d}}}{2}\right)
\end{aligned}
$$

Moreover, we also show in Appendix 2 that $P$, as a function of $K$ and $X$, writes:

$$
P=\frac{\left(\frac{1+\gamma(1-\alpha)}{(2-\alpha) \bar{\kappa}}\left(\left(\frac{2-\alpha}{1+\gamma(1-\alpha)}\right)^{2}-1\right)\right)^{\frac{1}{1-\gamma}} \exp \left(-\frac{(1-\alpha)|X|}{d(1+\gamma(1-\alpha))}\right)}{\left(\frac{K}{\langle K\rangle_{X}}\right)^{\frac{\alpha}{1+\gamma}}\left(\left(\frac{1-h \exp \left(-\frac{|X|}{d}\right)\left(1-\frac{\cosh \frac{X}{d}}{\exp \left(\frac{1}{d}\right)}\right)}{1-h \exp \left(-\frac{1}{2 d}\right)\left(1-\frac{\exp \left(-\frac{1}{2 d}\right)}{2}\right)}\right)\right)^{1+\gamma(1-\alpha)}}
$$

Once the price level associated to $K$ and $X$ is found, the potential (20) becomes:

$$
\frac{\omega^{2}}{\sigma^{2}} \int \Psi^{\dagger}(K, X, \theta)\left(K-\langle K\rangle_{X}\right)^{2} \Psi(K, X, \theta)
$$

with:

$$
\omega=\sigma \sqrt{\delta^{2}+\frac{\bar{A}^{2} A^{2}}{\left(A^{2}\left(1-h\left(1-\exp \left(-\frac{1}{d}\right)\right)\right)^{2}+\bar{A}^{2}\right)^{2}}}
$$

and the effective action, is now equal to:

$$
\begin{aligned}
S(\Psi)= & \int \Psi^{\dagger}(K, X, \theta)\left(-\sigma^{2} \nabla_{K}^{2}-\sigma_{X}^{2} \nabla_{X}^{2}-\vartheta^{2} \nabla_{\theta}^{2}+\frac{\omega^{2}}{\sigma^{2}}\left(K-\langle K\rangle_{X}\right)^{2}+\frac{1}{\vartheta^{2}}+\alpha\right) \Psi(K, X, \theta) \\
& +\int\left(V_{0}(X)\right)|\Psi(K, X, \theta)|^{2}+\int V_{1}\left(D_{12}\right)\left|\Psi\left(K_{1}, X_{1}, \theta\right)\right|^{2}\left|\Psi\left(K_{2}, X_{2}, \theta\right)\right|^{2} \\
& +\int V_{2}\left(D_{12}, D_{13}, D_{23}\right)\left|\Psi\left(K_{1}, X_{1}, \theta\right)\right|^{2}\left|\Psi\left(K_{2}, X_{2}, \theta\right)\right|^{2}\left|\Psi\left(K_{3}, X_{3}, \theta\right)\right|^{2}
\end{aligned}
$$

This simplified form will be useful to compute the transition functions of the agents in the system.

We can now interpret these first set of results. From the above, we can see that the average level of capital is a function of the variable $X$ and the parameter $d$. The average level of capital of an agent (25) decreases exponentially as a function of his distance from the center $X$. Indeed, an agent at the center has more opportunities to exchange. He faces a higher demand, has a higher price, a higher income, and accumulates more capital. Our results show that capital accumulation is an increasing function of parameter $d$, the average distance of interactions with other agents. Recall that agents exchange with all other agents, but that due to the exponential form of consumption an agent exchanges mainly with the agents on an interval of length $d$, centered around his position. The more the agent can exchange - the better the infrastructures for instance - the higher capital accumulation. Besides, inspection of (27) shows that the fluctuations of prices around the market clearing condition deteriorate the average level of capital stock.

Finally, and as expected, equation (25) shows that high capital productivity favors capital accumulation while higher rates of capital depreciation reduce capital stock.

Moreover, the above results show that the level of prices is a function of the variables $K, X$, and the parameter $d$. The price (29) of a good produced by an agent in a specific position $X$ is a decreasing function of the ratio between the level of capital and the level of average capital in $X$. We retrieve the result that the higher the level of capital, the more agents produce and sell at lower prices. However, this dependence to the level of capital is relative. Rather, it is the ratio of the agent's level of capital to the average level of 
capital that is determinant. The price (29) is also an exponentially decreasing function of position $X$. Given a constant level of capital, an agent at the periphery faces a lower demand and has lower prices than at the center of the exchange space. Ultimately, the price of a good produced is an increasing function of the parameter $d$. The higher $d$, the higher the volume of echanges, and the higher the prices.

\subsubsection{Phase 2: $\rho>0$}

In phase 2, computations are similar to phase 1 but expectations are computed for a field $\Psi_{0}(Z, \theta)+\Psi(Z, \theta)$. We thus compute the corrections to phase 1 due to the non-trivial $\Psi_{0}(Z, \theta)$.

Appendix 3 shows that the previous equations defining the potential (201), $P(22)$ and $\langle K\rangle_{X}$ are still valid:

$$
P=F(K, X)=K^{-\frac{\alpha}{1+\gamma}} f(X)
$$

However, now the trial function $f$ satisfies:

$$
\left(f\left(X_{1}\right)\right)^{1+\gamma}=\kappa \int\left(K_{2}\right)^{\frac{\alpha \gamma}{1+\gamma}}\left(K_{3}\right)^{-\frac{\alpha}{1+\gamma}} f\left(X_{3}\right) f\left(X_{2}\right) \exp \left(-\left(\frac{D_{12}+D_{23}}{d}\right)\right)\left|\bar{\Psi}\left(K_{3}, X_{3}\right)\right|^{2}\left|\bar{\Psi}\left(K_{2}, X_{2}\right)\right|^{2}
$$

with $\bar{\Psi}(K, X, \theta)=\Psi_{0}(K, X, \theta)+\Psi(K, X, \theta)$. Equation (33) is actually equation (24), but evaluated now for a "translated field", since the properties of $f\left(X_{1}\right)$ are modified by the non-trivial fundamental state. As explained above, this reflects the particular features of the non-trivial phase and the collective contribution of the environment created by the set of all agents.

Appendix 3 computes the function $f$ in two steps corresponding to the decomposition $\bar{\Psi}(K, X, \theta)=$ $\Psi_{0}(K, X, \theta)+\Psi(K, X, \theta)$. We find:

with:

$$
f(X)=D \exp \left(-\frac{|X|}{1+\gamma}\right)
$$

$$
\begin{aligned}
D= & \left(\frac{A}{\delta}\left(1-h \exp \left(-\frac{1}{2 d}\right)\left(1-\frac{\exp \left(-\frac{1}{2 d}\right)}{2}\right)\right)\right)^{\frac{\alpha}{(1-\alpha)(\gamma+1)}} \\
& \times\left(\frac{1+\gamma(1-\alpha)}{(2-\alpha) \bar{\kappa} \rho^{4}}\left(\left(\frac{2-\alpha}{1+\gamma(1-\alpha)}\right)^{2}-1\right)\right)^{\frac{1+\gamma(1-\alpha)}{\left(1-\gamma^{2}\right)(1-\alpha)}}
\end{aligned}
$$

and where $h$ is defined as in (27). The associated average capital stock is given by:

$$
\langle K\rangle_{X}=\left(\frac{A}{\delta} f(X)\left(1-h \exp \left(-\frac{|X|}{d}\right)\left(1-\frac{\cosh \frac{X}{d}}{\exp \left(\frac{1}{d}\right)}\right)\right)\right)^{\frac{1+\gamma}{1+\gamma(1-\alpha)}}
$$

with $h$ is defined as in (27). Moreover, we also show in Appendix 3 that $P$, as a function of $K$ and $X$, writes:

$$
P=\frac{\left(\frac{1+\gamma(1-\alpha)}{(2-\alpha) \bar{\kappa} \rho^{4}}\left(\left(\frac{2-\alpha}{1+\gamma(1-\alpha)}\right)^{2}-1\right)\right)^{\frac{1}{1-\gamma}} \exp \left(-\frac{(1-\alpha)|X|}{d(1+\gamma(1-\alpha))}\right)}{\left(\frac{K}{\langle K\rangle_{X}}\right)^{\frac{\alpha}{1+\gamma}}\left(\left(\frac{1-h \exp \left(-\frac{|X|}{d}\right)\left(1-\frac{\cosh \frac{X}{d}}{\exp \left(\frac{1}{d}\right)}\right)}{1-h \exp \left(-\frac{1}{2 d}\right)\left(1-\frac{\exp \left(-\frac{1}{2 d}\right)}{2}\right)}\right)\right)^{\frac{\alpha}{1+\gamma(1-\alpha)}}}
$$

Having found $\langle K\rangle_{X}$, appendix 3 shows that the potential part involving $K$ has the same form as in the first phase (30):

$$
\frac{\omega^{2}}{\sigma^{2}} \int \Psi^{\dagger}(K, X, \theta)\left(K-\langle K\rangle_{X}\right)^{2} \Psi(K, X, \theta)
$$

These results are similar to those of phase 1. The patterns of (35) and (34) as a function of $K, X$ and $d$, are the same as those of (29) et (25). Only their magnitudes differ. This is due to $\rho^{2}$, the norm of the 
fundamental state $\Psi_{0}$, a parameter specific to phase 2 . The two phases will be compared in our interpretation of the results. The formula for the effective action in phase 2 is also the same as (31). Yet again, in phase 2 , these formulas depend explicitly on the value of $\rho^{2}$. We previously found an approximative value for $\rho$. With the results now at hand, the value of $\rho$ can be found more precisely by writing the equation for the state $\Psi_{0}(K, X, \theta)$. Appendix 3 shows that:

$$
\rho^{2}=\frac{\kappa_{1}+\sqrt{\kappa_{1}^{2}-2 \kappa_{2}\left(2 \alpha+\sqrt{\kappa_{0}}+\sigma \delta\right)}}{2 \kappa_{2}}
$$

and the precise form of $\Psi_{0}(K, X, \theta)$ is given by:

$$
\Psi_{0}(K, X, \theta)=\rho N \Psi_{0}(K) \Psi_{0}(X)
$$

where:

$$
\begin{aligned}
& N=\frac{1}{2 \pi \sqrt{\kappa_{0}^{-\frac{1}{2}} \sigma \sqrt{\delta^{2}+\frac{\bar{A}^{2} A^{2}}{\left(A^{2}\left(1-h\left(1-\exp \left(-\frac{1}{d}\right)\right)\right)^{2}+\bar{A}^{2}\right)^{2}}}}} \\
& \rho^{2}=\frac{\kappa_{1}+\sqrt{\kappa_{1}^{2}-2 \kappa_{2}\left(2 \alpha+\frac{2}{\vartheta^{2}}+\sqrt{\kappa_{0}}+\sigma \delta\right)}}{2 \kappa_{2}} \\
& \Psi_{0}(K)=\exp \left(-\frac{\sqrt{\delta^{2}+\frac{\bar{A}^{2} A^{2}}{\left(A^{2}\left(1-h\left(1-\exp \left(-\frac{1}{d}\right)\right)\right)^{2}+\bar{A}^{2}\right)^{2}}}\left(K-\langle K\rangle_{X}\right)^{2}}{2 \sigma}\right) \\
& \Psi_{0}(X)=\exp \left(-\frac{\kappa_{0}^{-\frac{1}{2}}(X+\delta X)^{2}}{2}\right) H(x)+\exp \left(-\frac{\kappa_{0}^{-\frac{1}{2}}(X-\delta X)^{2}}{2}\right) H(-x) \\
& \delta X=\left(\chi_{1} \kappa_{1} \frac{K}{2\langle K\rangle} \rho^{2}-\chi_{2} \kappa_{2} \rho^{4}\right)
\end{aligned}
$$

\subsection{Transition probabilities}

In the previous paragraph, replacing $P$ as a function of $K, X$ and the field has reduced the system to a field theory of the two variables $K$ and $X$ with quadratic potentials (30) and (36). This simplification allows to compute the transition probabilities for the system in both phases, i.e. the probabilities for agents to evolve from one initial state of $K, X$, and $P$ towards a final state during a certain time-span, given the environment created by the set of $N$ agents (see Gosselin, Lotz, Wambst 2018). To do so, we first simplify in each phase the part of the action dependent on $X$.

\subsubsection{Phase 1: $\rho=0$}

Using the effective action (31), we can isolate the part of the action dependent on $X$ :

$$
\begin{aligned}
S_{2}(\Psi)= & \sigma_{X}^{2} \int|\nabla \Psi(Z, \theta)|^{2}+\int V_{0}(X)|\Psi(Z, \theta)|^{2}+\int V_{1}\left(D_{12}\right)\left|\Psi\left(Z_{1}, \theta\right)\right|^{2}\left|\Psi\left(Z_{2}, \theta\right)\right|^{2} \\
& +\int V_{2}\left(D_{12}, D_{13}, D_{23}\right)\left|\Psi\left(Z_{1}, \theta\right)\right|^{2}\left|\Psi\left(Z_{2}, \theta\right)\right|^{2}\left|\Psi\left(Z_{3}, \theta\right)\right|^{2}
\end{aligned}
$$

This action $S_{2}(\Psi)$ can be evaluated replacing the interaction potentials by their average in phase 1:

$$
\begin{aligned}
& -\int \frac{\kappa_{1}}{2}|\Psi(K, X, \theta)|^{2} \frac{K K^{\prime} \exp \left(-\chi_{1}|x-y|\right)}{\langle K\rangle^{2}}\left|\Psi\left(K^{\prime}, Y, \theta\right)\right|^{2} \\
\rightarrow & -\kappa_{1} \int\left\langle\frac{K K^{\prime} \exp \left(-\chi_{1}|x-y|\right)}{\langle K\rangle^{2}}\left|\Psi\left(K^{\prime}, Y, \theta\right)\right|^{2}\right\rangle|\Psi(K, X, \theta)|^{2}
\end{aligned}
$$


and:

$$
\begin{aligned}
& \int \frac{\kappa_{2}}{3}|\Psi(K, X, \theta)|^{2} \exp \left(-\chi_{2}|X-Y|-\chi_{2}|X-Z|-\chi_{2}|Y-Z|\right)\left|\Psi\left(K^{\prime}, Y, \theta\right)\right|^{2}\left|\Psi\left(K^{\prime \prime}, Z, \theta\right)\right|^{2} \\
\rightarrow & \kappa_{2} \int|\Psi(K, X, \theta)|^{2}\left\langle\exp \left(-\chi_{2}|X-Y|-\chi_{2}|X-Z|-\chi_{2}|Y-Z|\right)\left|\Psi\left(K^{\prime}, Y, \theta\right)\right|^{2}\left|\Psi\left(K^{\prime \prime}, Z, \theta\right)\right|^{2}\right\rangle
\end{aligned}
$$

The computations, performed in Appendix 4, lead to an approximation:

$$
S_{2}(\Psi)=-\sigma_{X}^{2} \Psi^{\dagger}(x) \nabla_{X}^{2} \Psi(x)+\omega_{X} \Psi^{\dagger}(x) X^{2} \Psi(x)+\alpha_{X} \Psi^{\dagger}(x) \Psi(x)
$$

with:

$$
\begin{aligned}
\omega_{X} & =\kappa_{0}+\kappa_{1} \frac{K}{\langle K\rangle} \exp \left(-\chi_{1}\right) \chi_{1}-\kappa_{2} \exp \left(-\chi_{2}\right) \chi_{2} \\
\alpha_{X} & =\alpha-\kappa_{1} \frac{K}{\langle K\rangle} \frac{2\left(1-\exp \left(-\chi_{1}\right)\right)}{\chi_{1}}+\kappa_{2} \frac{2\left(1-\exp \left(-\chi_{2}\right)\right)}{\chi_{2}}
\end{aligned}
$$

Gathering the part of the effective action depending on $K$ and our simplification for $S_{2}(\Psi)$, the system is thus described by an overall action:

$$
S(\Psi)=\Psi^{\dagger}(K, X, \theta)\left(-\sigma^{2} \nabla_{K}^{2}-\vartheta^{2} \nabla_{\theta}^{2}-\sigma_{X}^{2} \nabla_{X}^{2}+\frac{\omega^{2}}{\sigma^{2}}\left(K-\langle K\rangle_{X}\right)^{2}+\omega_{X} X^{2}+\frac{1}{\vartheta^{2}}+\alpha_{X}\right) \Psi(K, X, \theta)
$$

The equation (39) describes a system that looks like a three-variable system with quadratic potential. An important difference however is that each variable of the system influences the other non-linearly.

To get a better insight on the dynamics, we will simplify the mechanism at hand, and consider that when an agent moves from $K, X$ towards $K^{\prime}, X^{\prime}$, we will average along the path the influence of one variable on the other. To do so, we first set for two values $X$ and $X^{\prime}$ :

$$
\langle K\rangle=\frac{\langle K\rangle_{X}+\langle K\rangle_{X^{\prime}}}{2}
$$

where $\langle K\rangle$ is an average of capital for agents whose trajectory in the exchange space starts from $X$ to reach $X^{\prime}$. This represents the average influence of $\mathrm{X}$ on the capital stock within the dynamics.

Similarly, we define average values for $\omega_{X}$ and $\alpha_{X}$ along a path of capital from $K$ to $K^{\prime}$.

$$
\begin{aligned}
& \bar{\omega}_{X}=\kappa_{0}+\kappa_{1} \frac{\kappa_{1}}{2}\left(\frac{K}{\langle K\rangle_{X}}+\frac{K^{\prime}}{\langle K\rangle_{Y}}\right) \exp \left(-\chi_{1}\right) \chi_{1}-\kappa_{2} \exp \left(-\chi_{2}\right) \chi_{2} \\
& \bar{\alpha}_{X}=\alpha-\kappa_{1} \frac{\kappa_{1}}{2}\left(\frac{K}{\langle K\rangle_{X}}+\frac{K^{\prime}}{\langle K\rangle_{Y}}\right) \frac{2\left(1-\exp \left(-\chi_{1}\right)\right)}{\chi_{1}}+\kappa_{2} \frac{2\left(1-\exp \left(-\chi_{2}\right)\right)}{\chi_{2}}
\end{aligned}
$$

Once (39) found and given (40) and (41), the transition probability for one agent in the first phase can be computed. Recall that this is the probability for an agent to evolve from an initial state to a final state during a certain timespan. Appendix 4 shows that: 


$$
\begin{aligned}
& G\left(K, K^{\prime}, P, P^{\prime}, X, X^{\prime}, \theta, \theta^{\prime}\right) \\
& =\exp \left(-\left[\frac{\left(K-\langle K\rangle_{X}\right)^{2}}{2 \sigma^{2}}{ }_{(K, X)}^{\left(K^{\prime}, X^{\prime}\right)}\right]_{(K, X)}^{\left(K^{\prime}, X^{\prime}\right)}\right) \\
& \times \sqrt{\frac{\bar{\omega}_{X}}{2 \pi \sinh \left(\bar{\omega}_{X} t\right)}} \exp \left(-\frac{\bar{\omega}_{X}\left(\left(X^{2}+\left(X^{\prime}\right)^{2}\right) \cosh \left(\bar{\omega}_{X} t\right)-2 X X^{\prime}\right)}{2 \sinh \left(\bar{\omega}_{X} t\right)}\right) \\
& \times \sqrt{\frac{\omega}{2 \pi \sigma^{2} \sinh (\omega t)}} \exp \left(-\frac{\omega\left(\left((K-\langle K\rangle)^{2}+\left(K^{\prime}-\langle K\rangle\right)^{2}\right) \cosh (\omega t)-2(K-\langle K\rangle)\left(K^{\prime}-\langle K\rangle\right)\right)}{2 \sigma^{2} \sinh (\omega t)}\right) \\
& \times \delta\left(P-\frac{\left(\frac{1+\gamma(1-\alpha)}{(2-\alpha) \kappa\left(1-h \frac{A^{2}}{A^{2}}\left(1-\exp \left(-\frac{1}{d}\right)\right)\right) d}\left(\left(\frac{2-\alpha}{1+\gamma(1-\alpha)}\right)^{2}-1\right)\right)^{\frac{1}{1-\gamma}} \exp \left(-\frac{(1-\alpha)|X|}{1+\gamma(1-\alpha)}\right)}{\left(\frac{K}{\langle K\rangle_{X}}\right)^{\frac{\alpha}{1+\gamma}}\left(1-h \exp (-|X|)\left(1-\frac{\cosh X}{e}\right)\right)^{\frac{\alpha}{1+\gamma}}}\right) \\
& \times \delta\left(P^{\prime}-\frac{\left(\frac{1+\gamma(1-\alpha)}{(2-\alpha) \kappa\left(1-h \frac{A^{2}}{A^{2}}\left(1-\exp \left(-\frac{1}{d}\right)\right)\right) d}\left(\left(\frac{2-\alpha}{1+\gamma(1-\alpha)}\right)^{2}-1\right)\right)^{\frac{1}{1-\gamma}} \exp \left(-\frac{(1-\alpha)\left|X^{\prime}\right|}{1+\gamma(1-\alpha)}\right)}{\left(\frac{K^{\prime}}{\langle K\rangle_{X^{\prime}}}\right)^{\frac{\alpha}{1+\gamma}}\left(1-h \exp \left(-\left|X^{\prime}\right|\right)\left(1-\frac{\cosh X^{\prime}}{e}\right)\right)^{\frac{\alpha}{1+\gamma}}}\right) H\left(\theta-\theta^{\prime}\right)
\end{aligned}
$$

where $\delta(u)$ is the Dirac function. A Dirac function is a function which is null for all $u \neq 0$ and peaked at $u=0$.

Equation (42) computes the probability for an agent starting at $(K, P, X, \theta)$ to reach $\left(K^{\prime}, P^{\prime}, X^{\prime}, \theta^{\prime}\right)$ during a timespan $\theta-\theta^{\prime}$.

This formula also allows to compute the probability for $k$ agents with initial state $\left(K_{i}, P_{i}, X_{i}, \theta\right)_{i=1, \ldots, N}$ to reach the state $\left(K_{i}^{\prime}, P_{i}^{\prime}, X_{i}^{\prime}, \theta^{\prime}\right)_{i=1, \ldots, N}$. Formula (42) is the product of Green functions:

$$
\prod_{i=1}^{k} G\left(K_{i}, K_{i}^{\prime}, P_{i}, P_{i}^{\prime}, X_{i}, X_{i}^{\prime}, \theta, \theta^{\prime}\right)
$$

This probability is a product of independent probabilities. Indeed, recall that in this set up interactions have been absorbed in the Green function parameters. This illustrates the fact that the transition functions of agents are shaped by the global environment, and not by some specific interactions.

Formula (42) allows to understand the main features of individual agents' dynamics.

Consider an agent starting from $K^{\prime}, X^{\prime}$ and arriving at a final point $K, X$ after some time. This final point is partly random, but due to the parameters of the system some values of $K, X$ are more likely, and some dynamic patterns appear.

We will in turn examine each of the terms of this equation (42).

The first exponential in (42) is trivial: it merely translates the fact that the capital stock of agents in position $X$ is bounded around the average stock $\langle K\rangle_{X}$.

The second exponential in Formula (42) describes the dynamics on $X$. It has the form of a stochastic harmonic oscillator, with one important difference: the frequency $\bar{\omega}_{X}$ is not constant and satisfies (41). This exponential can be rewritten:

$$
\exp \left(-\frac{\bar{\omega}_{X}\left(\left(\left(X-X^{\prime}\right)^{2}\right)+\left(\cosh \left(\bar{\omega}_{X} t\right)-1\right)\left(X^{2}+\left(X^{\prime}\right)^{2}\right)\right)}{2 \sinh \left(\bar{\omega}_{X} t\right)}\right)
$$


The first term in the exponential $\left(X-X^{\prime}\right)^{2}$ represents a Brownian random walk: any agent starting at $X^{\prime}$ could move randomly with a standard deviation of $\sqrt{\frac{\sinh \left(\bar{\omega}_{X} t\right)}{\bar{\omega}_{X}}}$.

The additional term however, $\left(\cosh \left(\bar{\omega}_{X} t\right)-1\right)\left(X^{2}+\left(X^{\prime}\right)^{2}\right)$, favors a final $X$ closer from the center $X=0$ : the cohesion force drives agents toward the center of the exchange space.

The parameter $\bar{\omega}_{X}$ is a rough estimator of the speed of this move towards the center.

For $\bar{\omega}_{X}<<1$, the weight (43) is dampened for all final value of $X$, which means that all final points $X$ is equally probable. The driving force is weak, and the convergence very slow.

Inspection of (41) shows that this speed $\bar{\omega}_{X}$ depends on the agent's initial level of capital. As expected, this speed also depends positively on $\kappa_{0}$, the magnitude of the cohesion force, and negatively on $\kappa_{2}$, the magnitude of the repulsive force.

Most importantly $\kappa_{1}$, the magnitude of the attractive force is dampened by the ratio of the agent's current capital stock to $\langle K\rangle_{X}$. Recall that $\langle K\rangle_{X}$ is the average capital stock of agent's current - transitory - exchange position. Since $\langle K\rangle_{X}$ increases as the agent moves towards the center of the exchange space ( $X \rightarrow 0$ ), the closer the agent gets to the center, the more he decelerates. A strong enough repulsive force and a relatively low initial level of capital may prevent the agent to ever reach the center of the exchange space. The attractive force towards the center is thus inequal among agents. It favors those endowed with a higher initial capital.

The third exponential in (42) determines the dynamics on $K$. It can be rewritten:

$$
\exp \left(-\frac{\omega\left(\left(\left(K-K^{\prime}\right)^{2}\right)+(\cosh (\omega t)-1)\left((K-\langle K\rangle)^{2}+\left(K^{\prime}-\langle K\rangle\right)^{2}\right)\right)}{2 \sigma^{2} \sinh (\omega t)}\right)
$$

where $\langle K\rangle$ is defined by (40). For a relatively stable $X$, an agent's capital stock is in average driven towards the average stock of his exchange position. The quadratic term $\left(K-K^{\prime}\right)^{2}$ represents a random Brownian walk around this trend.

Equation (25) shows that as long as an agent moves towards the center his average capital stock will increase in average.

As a matter of fact, the two dynamics of $X$ and $K$ interact and can create a virtuous circle. A move towards the center tends to increase capital, and an increase of capital tends to accelerate the move towards the center. This positive interaction will only stop when the repulsive force will overcome the attractive forces. Given (41), this occurs when initial capital is insufficient. The attractive force depends on the ratio of initial over average capital. It may also occur if an adverse shock reduces the stock of capital. The agent can then be driven back towards the periphery.

The fourth term in equation (42) describes the market clearing conditions given $X$ and $K$. The Dirac function in the last line of (42) implements (29). For a given level of capital $K$, prices are higher towards the center of the exchange space, and for a given position in the exchange space $X$, prices are a decreasing function of $K$.

\subsubsection{Phase 2: $\rho>0$}

As in the non-trivial phase, we isolate in the effective action (31) the part dependent on $X$. Appendix 4 shows that in phase 2 its second order approximation is:

$$
\begin{aligned}
& S_{2}(\Psi)=\Psi^{\dagger}(K, X, \theta)\left(-\nabla_{X}^{2}+\kappa_{0}\left(1-\frac{\chi_{1}^{2} \kappa_{1} \rho^{2}}{2}+2 \chi_{2} \kappa_{2} \rho^{4}\right)(X+\operatorname{sgn}(X) \delta X)^{2}\right) \Psi(K, X, \theta) \\
& +\left(\rho^{4} \kappa_{2}-\rho^{2} \kappa_{1}-(\delta X)^{2}\right)|\Psi(K, X, \theta)|^{2}
\end{aligned}
$$

with:

$$
\rho^{2}=\frac{\kappa_{1}+\sqrt{\kappa_{1}^{2}-2 \kappa_{2}\left(2 \alpha+\sqrt{\kappa_{0}}+\sigma \delta\right)}}{2 \kappa_{2}}
$$


and:

$$
\delta X=\chi_{1} \kappa_{1} \frac{K}{2\langle K\rangle} \rho^{2}-\chi_{2} \kappa_{2} \rho^{4}
$$

Thus, the full action for the system is:

$$
\begin{aligned}
& S(\Psi)=\int \Psi^{\dagger}(K, X, \theta)\left(-\sigma^{2} \nabla_{K}^{2}-\vartheta^{2} \nabla_{\theta}^{2}-\sigma_{X}^{2} \nabla_{X}^{2}+\frac{\omega^{2}}{\sigma^{2}}(K-\langle K\rangle)^{2}\right) \Psi(K, X, \theta) \\
& +\kappa_{0} \int\left(\left(X+\chi \operatorname{sgn}(X)\left(\kappa_{1} \frac{K}{2\langle K\rangle} \rho^{2}-\kappa_{2} \rho^{4}\right)\right)^{2}+\alpha_{X}\right)|\Psi(K, X, \theta)|^{2} \\
& +\left(2 \rho^{2} \kappa_{2}-\kappa_{1}\right)\left|\int \Psi^{\dagger}(K, X, \theta) \Psi_{0}(K, X, \theta)\right|^{2}
\end{aligned}
$$

where:

$$
\begin{aligned}
\omega & =\sigma \sqrt{\delta^{2}+\frac{\bar{A}^{2} A^{2}}{\left(A^{2} U^{2}+\bar{A}^{2}\right)^{2}}} \\
\alpha_{X} & =-\frac{1}{2} \kappa_{0}^{\frac{1}{2}} \sqrt{1-\frac{\chi_{1}^{2} \kappa_{1} \rho^{2}}{2}+2 \chi_{2}^{2} \kappa_{2} \rho^{4}}-\frac{\sigma \sqrt{\delta^{2}+\frac{\bar{A}^{2} A^{2}}{\left(A^{2} U^{2}+\bar{A}^{2}\right)^{2}}}}{2}
\end{aligned}
$$

Given (48), the transition function in the phase $\rho \neq 0$ :

$$
\begin{aligned}
& G\left(K, K^{\prime}, P, P^{\prime}, X, X^{\prime}, \theta, \theta^{\prime}\right) \\
& =\exp \left(-\left[{\frac{\left(K-\langle K\rangle_{X}\right)^{2}}{2 \sigma^{2}}}_{(K, X)}^{\left(K^{\prime}, X^{\prime}\right)}\right]_{(K, X)}^{\left(K^{\prime}, X^{\prime}\right)}\right) \times G_{\frac{K+K^{\prime}}{2}}\left(X, X^{\prime}\right) \\
& \times \sqrt{\frac{\bar{\omega} / 2 \pi \sigma^{2}}{\sinh \left(\bar{\omega}\left(\theta-\theta^{\prime}\right)\right)}} \exp \left(-\frac{\bar{\omega}\left(\left((K-\langle K\rangle)^{2}+\left(K^{\prime}-\langle K\rangle\right)^{2}\right) \cosh \left(\bar{\omega}\left(\theta-\theta^{\prime}\right)\right)-2(K-\langle K\rangle)\left(K^{\prime}-\langle K\rangle\right)\right)}{2 \sigma^{2} \sinh \left(\bar{\omega}\left(\theta-\theta^{\prime}\right)\right)}\right) \\
& \times \delta\left(P-\frac{\left(\frac{1+\gamma(1-\alpha)}{(2-\alpha) \kappa\left(1-h \frac{A^{2}}{A^{2}}\left(1-\exp \left(-\frac{1}{d}\right)\right)\right) \rho^{4} d}\left(\left(\frac{2-\alpha}{1+\gamma(1-\alpha)}\right)^{2}-1\right)\right)^{\frac{1}{1-\gamma}} \exp \left(-\frac{(1-\alpha)|X|}{1+\gamma(1-\alpha)}\right)}{\left(\frac{K}{\langle K\rangle_{X}}\right)^{\frac{\alpha}{1+\gamma}}\left(1-h \exp (-|X|)\left(1-\frac{\cosh X}{e}\right)\right)^{\frac{\alpha}{1+\gamma}}}\right) \\
& \times \delta\left(P^{\prime}-\frac{\left(\frac{1+\gamma(1-\alpha)}{(2-\alpha) \kappa\left(1-h \frac{A^{2}}{A^{2}}\left(1-\exp \left(-\frac{1}{d}\right)\right)\right) \rho^{4} d}\left(\left(\frac{2-\alpha}{1+\gamma(1-\alpha)}\right)^{2}-1\right)\right)^{\frac{1}{1-\gamma}} \exp \left(-\frac{(1-\alpha)\left|X^{\prime}\right|}{1+\gamma(1-\alpha)}\right)}{\left(\frac{K^{\prime}}{\langle K\rangle_{X^{\prime}}}\right)^{\frac{\alpha}{1+\gamma}}\left(1-h \exp \left(-\left|X^{\prime}\right|\right)\left(1-\frac{\cosh X^{\prime}}{e}\right)\right)^{\frac{\alpha}{1+\gamma}}}\right) H\left(\theta-\theta^{\prime}\right)
\end{aligned}
$$

where:

$$
\begin{aligned}
G_{K}\left(X, X^{\prime}\right)= & \bar{G}\left(X+\delta X, X^{\prime}+\delta X\right) H(X) H\left(X^{\prime}\right)+\bar{G}\left(X-\delta X, X^{\prime}-\delta X\right) H(-X) H\left(-X^{\prime}\right) \\
& +\bar{G}\left(X+\delta X, X^{\prime}-\delta X\right) H(X) H\left(-X^{\prime}\right)+\bar{G}\left(X-\delta X, X^{\prime}+\delta X\right) H(-X) H\left(X^{\prime}\right)
\end{aligned}
$$

with:

$$
\bar{G}\left(X, X^{\prime}\right)=\sqrt{\frac{\kappa_{0}}{2 \pi \sigma_{X}^{2} \sinh \left(\kappa_{0} t\right)}} \exp \left(\left(-\frac{\kappa_{0}}{2 \sigma_{X}^{2} \sinh \left(\kappa_{0} t\right)}\right)\left(\left(X-X^{\prime}\right)^{2}+\left(\cosh \left(\kappa_{0} t\right)-1\right)\left(X^{2}+\left(X^{\prime}\right)^{2}\right)\right)\right)
$$

where $H(X)$ is the Heaviside function defined by $H(X)=1$ for $X>0$ and 0 otherwise. As in phase 1 , and for the same reasons, the definition of $\langle K\rangle$ is given by (40). The quantity $\delta X$ is defined in (47). 
Here again, the first exponential in (49) is trivial: it merely translates the fact that the capital stock of agents in position $X$ is bounded to be around the average stock $\langle K\rangle_{X}$.

The second exponential in formula (49) describes the dynamics on $X$. Given the formulas (49) and (50), this dynamic is different from that of phase 1. Equation (48) shows that an individual variable attraction point appears for each agent, along with $X=0$. This point depends both on the agent's initial position and capital.

The Green function $G_{\frac{K+K^{\prime}}{2}}\left(X, X^{\prime}\right)$ in (50) commands the dynamics of the exchange position. Assume $X$ and $X^{\prime}$ are positive. Using (51), one has:

$$
\begin{aligned}
G_{\frac{K+K^{\prime}}{2}}\left(X, X^{\prime}\right)= & \sqrt{\frac{\kappa_{0}}{2 \pi \sigma_{X}^{2} \sinh \left(\kappa_{0} t\right)}} \\
& \times \exp \left(\left(-\frac{\kappa_{0}}{2 \sigma_{X}^{2} \sinh \left(\kappa_{0} t\right)}\right)\left(\left(X-X^{\prime}\right)^{2}+\left(\cosh \left(\kappa_{0} t\right)-1\right)\left((X+\delta X)^{2}+\left(X^{\prime}+\delta X\right)^{2}\right)\right)\right)
\end{aligned}
$$

As in phase 1 , the quadratic term $\left(X-X^{\prime}\right)^{2}$ represents a random Brownian walk around the starting point $X^{\prime}$. The second quadratic term:

$$
\left(\cosh \left(\kappa_{0} t\right)-1\right)\left((X+\delta X)^{2}+\left(X^{\prime}+\delta X\right)^{2}\right)
$$

drives the position $X$ towards the position $-\delta X$. Thus, the attraction point is shifted from 0 to $-\delta X$.

For a large level of capital, equation (47) shows that the attraction point appears on the agent's opposite side of the center $X=0$. This variable attraction point quickly drives the agent towards $X=0$. When $X=0$ is reached, the agent does not cross this point in average. Were he to cross it randomly, he would be driven back towards $X=0$. Actually, when $X^{\prime}<0$ and $X<0$, i.e. when an agent has crossed the center $X=0$, equations (50) and (51) show that the agent is driven back toward the variable attraction point $\delta X>0$, which in turn drives back the agent toward 0 . The whole oscillatory process reveals that $X=0$ is indeed an attractive point.

For a low level of initial capital, the dynamics is different. The variable attraction point appears on the initial position side. The agent is driven towards this point, even if he was closer to the center $X=0$. This implies an eviction mechanism from the center for agents with low capital.

At first sight, the dynamics of capital accumulation should be identical to phase 1, since the third exponential in (49) is similar to (42). Up to some random fluctuations, agents are driven to the average level of capital of their current exchange position. However, since capital accumulation and exchange position are interacting, the features of the dynamics of $X$ in phase 2 impact capital accumulation.

In phase 1, a positive shock in capital could drive the agent towards the center, in turn increasing his capital stock.

Here, due to the presence of the variable attraction point, a small positive capital shock may not initiate a move towards the center. An agent with a low level of capital will be driven back towards his variable attraction point, possibly farther from the center.

The fourth term in equation (49) describes the market clearing conditions given $X$ and $K$. Actually, the Dirac function in the last line of (49) implements (35). For a given level of capital $K$, prices are higher towards the center of the exchange space, and for a given position in the exchange space $X$, prices are a decreasing function of $K$.

A last effect which is a direct consequence of the non-trivial phase cannot be read from the Green function (49) but can be tracked back to the last term of (48):

$$
\left(2 \rho^{2} \kappa_{2}-\kappa_{1}\right)\left|\int \Psi^{\dagger}(K, X, \theta) \Psi_{0}(K, X, \theta)\right|^{2}
$$

The effective action in phase 2 includes a positive term, since now $\rho^{2}$ is greater than $\frac{\kappa_{1}}{2 \kappa_{2}}$. Appendix 5 shows that this term induces a barrier between agents in position $X$. Agents with an initial capital stock below $\langle K\rangle_{X}$ will find it harder to accumulate above $\langle K\rangle_{X}$ and thus improve their exchange position while agents 
with $K>\langle K\rangle_{X}$ will more likely remain above this threshold. Therefore, in phase 2, a threshold effect appears at each point on the exchange space. Agents with high initial capital will more likely overcome barriers and accumulate, while the others will be evicted from positions in the exchange space for which their initial stock of capital is too low.

\section{Synthesis and discussion}

To interpret the previous results, recall that this model considers loosely defined variables. The exchange space for instance can be seen both as a geographical space, such as national markets or individual sectors, and as scale of terms of exchanges.

Three forces characterize this exchange space. The cohesion force is the only force that attracts all agents towards the center of the exchange space. The attractive and repulsive forces bring agents together or apart on the $X$ axis, respectively. Since exchanges are proportional to the distance between agents, the attractive forces facilitate exchanges between agents, whereas the repulsive force reduces competition, by preventing new entrants or more distant agents to establish or strengthen exchanges with consumers. These two forces, attractive and repulsive, always exist within a market, yet our work stresses that it is $r=\kappa_{2} / \kappa_{1}$, the relative magnitude of the repulsive force with respect to the attractive force, that matters. This ratio commands the occurrence of the two phases of the dynamics.

In phase $1, r$ is high and free exchange is limited. Markets are relatively protected, or exchanges may be prevented for other reasons, such as distance, lack of infrastructure, regulation, etc. The cohesion force improves in average the terms of exchange across all agents and contributes to homogenizing the agents. This tendency impacts all agents, even though agents with high initial capital are favored in the process. On the contrary, in phase 2, the repulsive force is relatively weaker, and $r$ is low. The agent will experience higher mobility within the exchange space.

The two phases present similarities. In both, the price of the producer and the average capital stock of agents decrease exponentially with the distance to the center. Agents at the periphery produce less and sell at a lower price than central agents. Yet dynamically the two phases are different. Phase 1 displays stability in exchanges, lower competition, a broad based although slow improvement in the terms of exchanges. In this phase, random shocks can redistribute capital and initiate a virtuous circle of capital accumulation.

In phase 2 , the weakness of the repulsive force induces greater instability in trade relations, but also greater competition among agents. Increased mobility favors capital accumulation for producers with high initial level of capital. Producers with a low level of initial capital will experience eviction from the exchange space and a deterioration in their prices and revenues. This is due to an indirect effect: increased exchanges induce greater competition and increased inequality in wealth distribution. Actually, removing the repulsive force opens market to agents with higher level of capital. Selling their products at a relatively low price, these agents gain market shares over their competitors. A producer that has penetrated a market experiences a higher demand, higher prices, a higher revenue, and in turn higher capital. This same mechanism, repeated over time, may drive him along the exchange space to a relatively dominant position.

On the contrary producers with low capital on an existing market will be progressively evicted. To them, market liberalization is an increase in neighboring competitors. They will face a lower demand, lose market shares and experience a decrease in income that will push them towards the periphery, and exclude them from their market. Such mechanisms are at play within sectors where liberalization favors agents with high concentration of capital. The arrival of big agricultural producers in Africa evicted local producers, that ended up selling their work force to their previous competitors, and in some cases, lead them to migrate.

Phase 2 displays a seemingly counter-intuitive result: except under mild market liberalization, i.e. when $r$ is in some range, average capital is lower in phase 2 than in phase 1 . This result is mainly due to our assumption of constant technology. Because of this assumption, our model is only valid within a relatively short period of time. Therefore, our conclusions do not contradict the standard accounts of industrial revolution. The short periods of liberalization during the technological boost of the nineteenth century did not lead decisively to higher accumulation, and freedom of exchanges has been at times contemporaneous of a crisis. Thus, our model sheds a nuanced light on free exchange dynamics. In our context, a high disparity in capital and thus in revenue does not imply an average improvement of wealth. Disparities in capital accumulation are directly related to a collapse in global demand and a lower average wealth. 
Our model has a last implication. In our setting, capital must be renewed to produce: when an agent cannot do so, he disappears as a producer within his market. This mechanism repeated over time may leave the agent with the sole capital he may renew, namely his labor force. Thus, phase 2 of our model also describes the evolution from a society with a large number of small producers towards a society with few capitalistic producers and a large number of workers: it accounts for de-homogenization of society.

\section{Conclusion}

In this paper, we have developed a model of capital accumulation with a large number of heterogeneous agents. This model keeps some features of the classical economic models - a standard equation of capital accumulation, production function, and market clearing condition. However, it includes an exchange space that dynamically interacts with capital accumulation. Besides, the classical description of the model is replaced by field theory techniques. Our results show that, depending on the parameters of the model, the system displays various phases, each describing different accumulation processes. Depending on the parameters of the model, capital accumulation is not necessarily favored by greater market liberalization. Besides, capital accumulation highly depends on each agent's initial conditions, and shows that the capital dynamics cannot be reduced to simple aggregates. Various dynamic patterns appear depending on agents. Yet our model enlightens the fact that by its dynamic divergences, a society of atomic producers can divide between groups of accumulating producers, and agents progressively losing their capital. Our results show a dynamic that can produce a society divided in two classes, the first one accumulating capital, the other one losing its capital, up to the point where its only capital left will be its labor. This model shows the advantages of field theory modeling in economics. It leads to a finer description of the agents' dynamics,

both at a global level and individual level, the two descriptions interacting permanently. Our future work will continue to explore this approach to other fields of economics. 


\section{Appendices}

\subsection{Appendix 0}

It will be useful for the description in term of field theory to rewrite the weight (15) in a different form. We introduce a variable $\tilde{X}_{i}(t)$ so that (15) is equal to:

$$
\begin{gathered}
\exp \left(-\sum_{i}\left(\int \frac{1}{2 \sigma^{2}}\left(\dot{K}_{i}(t)+\delta K_{i}(t)-A P_{i}(t) K_{i}^{\alpha}(t)+\tilde{X}_{i}(t)\right)^{2}\right.\right. \\
\left.\left.-\frac{\bar{A}^{2}}{2 \sigma^{2}}\left(P_{i}^{1+\gamma}(t) K_{i}^{\alpha}(t)-U_{2, i}\right)^{2}+\delta\left(\tilde{X}_{i}(t)-U_{1, i}\right)\right)\right)
\end{gathered}
$$

where $\delta\left(\tilde{X}_{i}(t)-U_{1, i}\right)$ is the delta function and:

$$
\begin{aligned}
U_{1 i} & =\kappa A P_{i}(t) K_{i}^{\alpha}(t) \sum_{j, k} \frac{P_{k}(t)}{P_{j}^{\gamma}(t)} \exp \left(-\frac{d_{i j}(t)+d_{i k}(t)}{d}\right) \\
U_{2, i} & =\kappa \sum_{j, k} P_{j}(t) K_{j}^{\alpha}(t) P_{k}(t) \exp \left(-\frac{d_{i j}(t)+d_{k j}(t)}{d}\right)
\end{aligned}
$$

The full weight of the system is thus:

$$
\begin{aligned}
& \exp \left(-\frac{\left(\dot{K}_{i}(t)+\delta K_{i}(t)-A P_{i}(t) K_{i}^{\alpha}(t)+\tilde{X}_{1}\right)^{2}}{2 \sigma^{2}}-\frac{\bar{A}^{2}\left(P_{i}^{1+\gamma}(t) K_{i}^{\alpha}(t)-U_{2}\right)^{2}}{2 \sigma^{2}}+\delta\left(\tilde{X}_{1}-U_{1}\right)\right) \\
& \times \exp \left(-\left(\sum_{i}\left(\frac{\left(\dot{X}_{i}(t)\right)^{2}}{\sigma_{X}^{2}}+V_{0}\left(X_{i}(t)\right)\right)+\sum_{i, j} V_{1}\left(d_{i j}(t)\right)+\sum_{i, j, k} V_{2}\left(d_{i j}(t), d_{i k}(t), d_{j k}(t)\right)\right)\right)
\end{aligned}
$$

\subsection{Appendix 1}

We use the techniques defined in Gosselin, Lotz and Wambst $(2017,2018)$ to switch from the probabilistic description of the model to the field theoretic formalism. A presentation can found in Gosselin, Lotz and Wambst (2018), but we recall here what is needed for our purpose.

The idea is the following. For a large number of agents, the system described by (52), involves a large number of variables $X(t)$ that are difficult to handle. We consider the space $H$ of complex functions defined on the space of a single agent's actions. The space $H$ describes the collective behavior of the system. Each function $\Psi$ of $H$ encodes a particular state of the system. Then, to each function $\Psi$ of $H$, we associate a statistical weight, i.e. a probability describing the state encoded in $\Psi$. This probability is written $\exp (-S(\Psi))$, where $S(\Psi)$ is a functional, i.e. a function of the function $\Psi$. The form of $S(\Psi)$ is derived directly from the form of (52).

The weight considered in this work is a particular case of those presented in Gosselin, Lotz and Wambst (2018), plus some adaptation. In Gosselin, Lotz and Wambst (2018), we showed that for a weight describing interaction between individual agents:

$$
\begin{aligned}
& \sum_{i} \int_{0}^{T}\left(\frac{1}{\sigma^{2}} \int_{0}^{T}\left(\frac{d}{d s} X_{i}(s)\right)^{2}+V_{1}\left(X_{s}^{(i)}\right)\right) d s+\frac{1}{\eta^{2}} \sum_{i} \int_{0}^{T}\left(\frac{d}{d t} X_{i}(t)-H\left(X_{i}(t)\right)\right)^{2} d t \\
& +\sum_{k \geqslant 2} \sum_{i_{1}, \ldots, i_{k}} \int_{0}^{T} \int_{0}^{T} \frac{V_{k}\left(X_{s_{1}}^{\left(i_{1}\right)}, \ldots, X_{s_{k}}^{\left(i_{k}\right)}\right)}{\theta^{2}} d s_{1} \ldots d s_{k}
\end{aligned}
$$


the following field action functional contains the same information about the system:

$$
\begin{aligned}
S(\Psi)= & \int\left(\Psi^{\dagger}(X)\left(-\sigma^{2} \nabla^{2}+V_{1}(X)+\alpha\right) \Psi(X)\right) d X-\sum_{i} \int \Psi^{\dagger}(X)\left(\eta^{2} \nabla^{2}+\nabla \cdot H(x)\right) \Psi(X) d X \\
& +\frac{1}{\theta^{2}} \sum_{k \geqslant 2} \sum_{k \geqslant 2} \int \Psi\left(X_{1}\right) \ldots \Psi\left(X_{k}\right) V_{k}\left(X_{1} \ldots X_{k}\right) \Psi^{\dagger}\left(X_{1}\right) \ldots \Psi^{\dagger}\left(X_{k}\right) d X_{1} \ldots d X_{k}
\end{aligned}
$$

where $\alpha$ is the parameter arising in the Laplace transform of the statistical weight (53), (see Gosselin, Lotz, Wambst 2017) and where $\Psi^{\dagger}(x)$ denotes the complex conjugate of $\Psi(x)$. The operator $\nabla$ is the gradient operator, a vector whose $i$-th coordinate is the first derivative $\frac{\partial}{\partial x_{i}}: \nabla=\left(\frac{\partial}{\partial X_{i}}\right)$. The operator $\nabla^{2}$ denotes the Laplacian:

$$
\nabla^{2}=\sum_{i} \frac{\partial^{2}}{\partial X_{i}^{2}}
$$

where the sum runs over the coordinates $x_{i}$ of the vector $x$. Applying this to our previous example, where $\Psi(X)=\Psi(c, k)$, we get $\nabla^{2}=\frac{\partial^{2}}{\partial c^{2}}+\frac{\partial^{2}}{\partial k^{2}}$.

Coming back to the model at stake here, and discarding for the moment the weight associated to the position in the exchange space, the statistical weight for the Price plus Capital part was (52):

$$
\exp \left(-\frac{1}{2 \sigma^{2}}\left(\dot{K}_{i}(t)+\delta K_{i}(t)-A P_{i}(t) K_{i}^{\alpha}(t)+U_{1 i}\right)^{2}-\frac{\bar{A}^{2}}{2 \sigma^{2}}\left(P_{i}^{1+\gamma}(t) K_{i}^{\alpha}(t)-U_{2, i}\right)^{2}\right)
$$

with:

$$
\begin{aligned}
U_{1 i} & =\kappa A P_{i}(t) K_{i}^{\alpha}(t) \sum_{j, k} \frac{P_{k}(t)}{P_{j}^{\gamma}(t)} \exp \left(-\frac{\left|X_{i}(t)-X_{j}(t)\right|}{d}-\frac{\left|X_{i}(t)-X_{k}(t)\right|}{d}\right) \\
U_{2 i} & =\kappa \sum_{j, k} P_{j}(t) K_{j}^{\alpha}(t) P_{k}(t) \exp \left(-\frac{\left|X_{i}(t)-X_{j}(t)\right|}{d}-\frac{\left|X_{k}(t)-X_{j}(t)\right|}{d}\right)
\end{aligned}
$$

The difference with (53) is that, here, the interactions between different agents (terms involving sums over different agents $j, k, \ldots$, are local, i.e. the quantities involved in these terms are considered at the same time, whereas it is not required in (53). As explained in Gosselin, Lotz and Wambst (2017), the introduction of local interactions can be done by introducing for each agent $i$, a counting variable $\Theta_{i}(s)$ which is roughly equal to $s$ apart some random fluctuation. A statistical weight has to be introduced for this counting variable $\Theta_{i}(s)$ and then, one has to replace this weight by its field counterpart:

$$
\int \exp \left(-\int \frac{\left(\dot{\Theta}_{i}(s)-1\right)^{2}}{2 \vartheta^{2}}\right) \mathcal{D} \Theta_{i}(s) \rightarrow \Psi^{\dagger}(K, P, \hat{P}, X, \theta) \nabla_{\theta} \cdot\left(\vartheta^{2} \nabla_{\theta}-2\right) \Psi(K, P, \hat{P}, X, \theta)
$$

We will now show that the overall action for the Price + Capital part associated to (52) writes:

$$
\begin{aligned}
& \Psi^{\dagger}(K, X, \theta)\left(-\sigma^{2} \nabla_{K}^{2}+\frac{\left(\delta K-A P K^{\alpha}\left(1-\hat{U}_{1}\right)\right)^{2}}{\sigma^{2}}+\sigma_{P}^{2}\left(\frac{A K^{\alpha+1}}{\sigma^{2}(\alpha+1)}\right)^{2}+\frac{\bar{A}^{2}\left(P^{1+\gamma} K^{\alpha}+U_{2}\right)^{2}}{\sigma^{2}}\right. \\
& \left.-\left(\sigma_{P}^{2} \nabla_{P}^{2}+\sigma_{X}^{2} \nabla_{X}^{2}\right)-\vartheta^{2} \nabla_{\theta}^{2}+\frac{1}{\vartheta^{2}}+\alpha\right) \Psi(K, X, \theta) \\
\simeq & \Psi^{\dagger}(K, X, \theta)\left(-\sigma^{2} \nabla_{K}^{2}+\frac{\left(\delta K-A P K^{\alpha}\left(1-\hat{U}_{1}\right)\right)^{2}}{\sigma^{2}}+\left(P^{1+\gamma} K^{\alpha}+U_{2}\right)^{2}-\sigma_{X}^{2} \nabla_{X}^{2}-\vartheta^{2} \nabla_{\theta}^{2}+\frac{1}{\vartheta^{2}}+\alpha\right) \Psi(K, X, \theta)
\end{aligned}
$$


which is in first approximation:

$\Psi^{\dagger}(K, X, \theta)\left(-\sigma^{2} \nabla_{K}^{2}+\frac{\left(\delta K-A P K^{\alpha}\left(1-\hat{U}_{1}\right)\right)^{2}}{\sigma^{2}}+\left(P^{1+\gamma} K^{\alpha}+U_{2}\right)^{2}-\sigma_{X}^{2} \nabla_{X}^{2}-\vartheta^{2} \nabla_{\theta}^{2}+\frac{1}{\vartheta^{2}}+\alpha\right) \Psi(K, X, \theta)$

with:

$$
\begin{aligned}
& \hat{U}_{1}=\kappa \int \frac{P_{3} \exp \left(-\left(\frac{D_{12}+D_{13}}{d}\right)\right)}{P_{2}^{\gamma}}\left|\bar{\Psi}\left(Z_{2}, \theta\right)\right|^{2}\left|\bar{\Psi}\left(Z_{3}, \theta\right)\right|^{2} \\
& U_{2}=-\kappa \int P_{2}\left(K_{2}\right)^{\alpha} P_{3} \exp \left(-\left(\frac{D_{12}+D_{23}}{d}\right)\right)\left|\Psi\left(Z_{2}, \theta\right)\right|^{2}\left|\Psi\left(Z_{3}, \theta\right)\right|^{2}
\end{aligned}
$$

To prove this result, we first start with an arbitrary weight with interactions between agents and including the counting variable $\Theta(s)$. Such a weight includes our model as a particular case.

$$
\exp \left(-\int \frac{\left(\dot{X}_{i}(t)+G\left(X_{i}(t)\right)-\sum_{j} V\left(X_{i}(t), X_{j}(t)\right)\right)^{2}}{2 \sigma^{2}}\right)
$$

This form describes the first part of (54) if we set:

$$
\begin{aligned}
G\left(X_{i}(t)\right) & =\delta K_{i}(t)-A P_{i}(t) K_{i}^{\alpha}(t) \\
V\left(X_{i}(t), X_{j}(t)\right) & =-U_{1}
\end{aligned}
$$

The second part of (54):

$$
-\frac{\bar{A}^{2}}{2 \sigma^{2}}\left(P_{i}^{1+\gamma}(t) K_{i}^{\alpha}(t)-U_{2, i}\right)^{2}
$$

has a direct equivalent in term of field theory:

$$
\Psi^{\dagger}(K, X, \theta)\left(\left(P^{1+\gamma} K^{\alpha}+U_{2}\right)^{2}\right) \Psi(K, X, \theta)
$$

so we skip it from the following argument and reintroduce it at the end of the proof.

Then, as in Gosselin, Lotz and Wambst (2017), we replace in $V\left(X_{i}(t), X_{j}(t)\right)$, the time by two independent parameters $t_{i}$ and $t_{j}$, and impose the equality of the counting variables associated to these parameters: $\theta_{j}\left(t_{j}\right)-\theta_{i}\left(t_{i}\right)=0$. Then the weight (54) rewrites:

$$
\rightarrow \exp \left(-\frac{\left(\dot{X}_{i}\left(t_{i}\right)+G\left(X_{i}\left(t_{i}\right)\right)-\sum_{j} \int V\left(X_{i}\left(t_{i}\right), X_{j}\left(t_{j}\right)\right) \delta\left(\theta_{j}\left(t_{j}\right)-\theta_{i}\left(t_{i}\right)\right)\right)^{2}}{2 \sigma^{2}}\right)
$$

To simplify the notations, and include possible generalizations, we will also replace:

$$
\begin{aligned}
\int V\left(X_{i}\left(t_{i}\right), X_{j}\left(t_{j}\right)\right) \delta\left(\theta_{j}\left(t_{j}\right)-\theta_{i}\left(t_{i}\right)\right) & \rightarrow \int V\left(X_{i}\left(t_{i}\right), \theta_{i}\left(t_{i}\right), X_{j}\left(t_{j}\right), \theta_{j}\left(t_{j}\right)\right) \\
& =\int V\left(Y_{i}\left(t_{i}\right), Y_{j}\left(t_{j}\right)\right)
\end{aligned}
$$

where we set:

$$
Y_{i}\left(t_{i}\right)=\left(X_{i}\left(t_{i}\right), \theta_{i}\left(t_{i}\right)\right)
$$

That is, the $\delta$ factor of the potential is replaced by an arbitrary function of the counting variables $\theta_{i}\left(t_{i}\right)$, $\theta_{j}\left(t_{j}\right)$. Then, we introduce an auxiliary variable $\tilde{X}_{i}\left(t_{i}\right)$, equal up to a random error of small square deviation $\sigma_{2}^{2}$, to $\sum_{j} \int V\left(Y_{i}\left(t_{i}\right), Y_{j}\left(t_{j}\right)\right)$ and the weight (56) becomes: 


$$
\begin{aligned}
& \exp \left(-\frac{\left(\dot{X}_{i}\left(t_{i}\right)+G\left(X_{i}\left(t_{i}\right)\right)-\sum_{j} \int V\left(Y_{i}\left(t_{i}\right), Y_{j}\left(t_{j}\right)\right)\right)^{2}}{2 \sigma^{2}}\right) \\
= & \exp \left(-\frac{\left(\dot{X}_{i}(t)+G\left(X_{i}\left(t_{i}\right)\right)-\tilde{X}_{i}\left(t_{i}\right)\right)^{2}}{2 \sigma^{2}}-\frac{\left(\tilde{X}_{i}\left(t_{i}\right)-\sum_{j} \int V\left(Y_{i}\left(t_{i}\right), Y_{j}\left(t_{j}\right)\right)\right)^{2}}{2 \sigma_{2}^{2}}\right) \\
= & \exp \left(-\frac{\left(\dot{X}_{i}(t)+G\left(X_{i}\left(t_{i}\right)\right)-\tilde{X}_{i}\left(t_{i}\right)\right)^{2}}{2 \sigma^{2}}\right. \\
& \left.-\frac{\left(\tilde{X}_{i}^{2}\left(t_{i}\right)\right)^{2}-2 \tilde{X}_{i}\left(t_{i}\right) \sum_{j} \int V\left(Y_{i}\left(t_{i}\right), Y_{j}\left(t_{j}\right)\right)+\sum_{j} \int V\left(Y_{i}\left(t_{i}\right), Y_{j}\left(t_{j}\right)\right) \sum_{j} \int V\left(Y_{i}\left(t_{i}\right), Y_{j}\left(t_{j}\right)\right)}{2 \sigma_{2}^{2}}\right)
\end{aligned}
$$

We also assume that:

$$
\sigma_{2}^{2}<<\sigma^{2}
$$

This weight has the required form to apply the techniques developped in Gosselin, Lotz and Wambst (2017), and the corresponding field theory action writes:

$$
\begin{aligned}
& \Psi^{\dagger}(Y, \tilde{X})\left(-\nabla_{\theta}\left(\vartheta^{2} \nabla_{\theta}-2\right)-\frac{1}{2} \nabla_{X}\left(\sigma^{2} \nabla_{X}+G(X)+\tilde{X}\right)+G^{\prime}(X)-\frac{1}{2} \varepsilon^{2} \nabla_{\tilde{X}}^{2}-\frac{1}{2} \omega \tilde{X}^{2}\right) \Psi(Y, \tilde{X}) \\
& +\int \frac{\tilde{X}^{2}}{2 \sigma_{2}^{2}}|\Psi(Y, \tilde{X})|^{2}-\int \frac{\tilde{X} V\left((Y, \tilde{X}),\left(Y^{\prime}, \tilde{X}^{\prime}\right)\right)}{\sigma_{2}^{2}}|\Psi(Y, \tilde{X})|^{2}\left|\Psi\left(Y^{\prime}, \tilde{X}^{\prime}\right)\right|^{2} \\
& +\int \frac{V\left((Y, \tilde{X}),\left(Y^{\prime}, \tilde{X}^{\prime}\right)\right) V\left((Y, \tilde{X}),\left(Y^{\prime \prime}, \tilde{X}^{\prime \prime}\right)\right)}{2 \sigma_{2}^{2}}|\Psi(Y, \tilde{X})|^{2}\left|\Psi\left(Y^{\prime}, \tilde{X}^{\prime}\right)\right|^{2}\left|\Psi\left(Y^{\prime \prime}, \tilde{X},\right)\right|^{2}
\end{aligned}
$$

We apply the transformation on the field

$$
\Psi(Y, \tilde{X})=\exp \left(-\int(G(X)+\tilde{X})\right) \bar{\Psi}(Y, \tilde{X})
$$

and change of notation for the sake of simplicity:

$$
\bar{\Psi}(Y, \tilde{X}) \rightarrow \Psi(Y, \tilde{X})
$$

For an action:

$$
\begin{aligned}
& \Psi^{\dagger}(Y, \tilde{X})\left(-\nabla_{\theta}\left(\vartheta^{2} \nabla_{\theta}-2\right)-\frac{1}{2} \nabla_{X}^{2}+(G(X)+\tilde{X})^{2}-\frac{1}{2} \varepsilon^{2} \nabla_{\tilde{X}}^{2}-\frac{1}{2} \omega \tilde{X}^{2}\right) \Psi(Y, \tilde{X}) \\
& +\int \frac{\tilde{X}^{2}}{2 \sigma_{2}^{2}}|\Psi(Y, \tilde{X})|^{2}-\int \frac{\tilde{X} V\left((Y, \tilde{X}),\left(Y^{\prime}, \tilde{X}^{\prime}\right)\right)}{\sigma_{2}^{2}}|\Psi(Y, \tilde{X})|^{2}\left|\Psi\left(Y^{\prime}, \tilde{X}^{\prime}\right)\right|^{2} \\
& +\int \frac{V\left((Y, \tilde{X}),\left(Y^{\prime}, \tilde{X}^{\prime}\right)\right) V\left((Y, \tilde{X}),\left(Y^{\prime \prime}, \tilde{X}^{\prime \prime}\right)\right)}{2 \sigma_{2}^{2}}|\Psi(Y, \tilde{X})|^{2}\left|\Psi\left(Y^{\prime}, \tilde{X}^{\prime}\right)\right|^{2}\left|\Psi\left(Y^{\prime \prime}, \tilde{X},\right)\right|^{2}
\end{aligned}
$$

For potential satisfying: $V\left((Y, \tilde{X}),\left(Y^{\prime}, \tilde{X}^{\prime}\right)\right)=V\left(Y, Y^{\prime}\right)$, which is the case considered in this work, it 
simplifies as:

$$
\begin{aligned}
& \Psi^{\dagger}(Y, \tilde{X})\left(-\nabla_{\theta}\left(\vartheta^{2} \nabla_{\theta}-2\right)-\frac{1}{2} \nabla_{X}^{2}+(G(X)+\tilde{X})^{2}-\frac{1}{2} \varepsilon^{2} \nabla_{\tilde{X}}^{2}-\frac{1}{2} \omega\right) \Psi(Y, \tilde{X}) \\
& +\int \frac{\tilde{X}^{2}}{2 \sigma_{2}^{2}}|\Psi(Y, \tilde{X})|^{2}-\int \frac{\tilde{X} V\left(Y, Y^{\prime}\right)}{\sigma_{2}^{2}}|\Psi(Y, \tilde{X})|^{2}\left|\Psi\left(Y^{\prime}, \tilde{X}^{\prime}\right)\right|^{2} \\
& +\int \frac{V\left(Y, Y^{\prime}\right) V\left(Y, Y^{\prime \prime}\right)}{2 \sigma_{2}^{2}}|\Psi(Y, \tilde{X})|^{2}\left|\Psi\left(Y^{\prime}, \tilde{X}^{\prime}\right)\right|^{2}\left|\Psi\left(Y^{\prime \prime}, \tilde{X} "\right)\right|^{2}
\end{aligned}
$$

and this is equal to:

$$
\begin{aligned}
& \Psi^{\dagger}(Y, \tilde{X})\left(-\nabla_{\theta}\left(\vartheta^{2} \nabla_{\theta}-2\right)-\frac{1}{2} \nabla_{X}^{2}+(G(X)+\tilde{X})^{2}-\frac{1}{2} \varepsilon^{2} \nabla_{\tilde{X}}^{2}-\frac{1}{2} \omega\right) \Psi(Y, \tilde{X}) \\
& +\int \frac{\left(\tilde{X}-\int V\left(Y, Y^{\prime}\right)\left|\Psi\left(Y^{\prime}, \tilde{X}^{\prime}\right)\right|^{2}\right)^{2}}{2 \sigma_{2}^{2}}|\Psi(Y, \tilde{X})|^{2}
\end{aligned}
$$

Now, given our assumptions: $\varepsilon^{2}<<1, \sigma_{2}^{2}<<1$, we can consider that: $\varepsilon^{2} \nabla_{\tilde{X}}^{2} \rightarrow 0$. As a consequence, the condition

$$
0=\int \Psi^{\dagger}(Y, \tilde{X})\left(\tilde{X}-\int V\left(Y, Y^{\prime}\right)\left|\Psi\left(Y^{\prime}, \tilde{X}^{\prime}\right)\right|^{2}\right)^{2} \Psi(Y, \tilde{X})
$$

imposed by $\sigma_{2}^{2}$ in (60) is obtained for a function of the type:

$$
\Psi(Y, \tilde{X})=\delta\left(\tilde{X}-\int \Psi^{\dagger}\left(Y^{\prime}\right) V\left(Y, Y^{\prime}\right) \Psi\left(Y^{\prime}\right)\right) \Psi(Y)
$$

The $\delta\left(\tilde{X}-\int \Psi^{\dagger}\left(Y^{\prime}\right) V\left(Y, Y^{\prime}\right) \Psi\left(Y^{\prime}\right)\right)$ is gaussian of norm equal to 1 peaked around $\int \Psi^{\dagger}\left(Y^{\prime}\right) V\left(Y, Y^{\prime}\right) \Psi\left(Y^{\prime}\right)$. As a consequence:

$$
\begin{aligned}
& \int \Psi^{\dagger}(Y, \tilde{X})(G(X)+\tilde{X})^{2} \Psi(Y, \tilde{X}) \\
= & \int \Psi^{\dagger}(Y, \tilde{X})\left(G(X)+\int V\left(Y, Y^{\prime}\right)\left|\Psi\left(Y^{\prime}, \tilde{X}^{\prime}\right)\right|^{2}\right)^{2} \Psi(Y, \tilde{X}) \\
& -2 \int \Psi^{\dagger}(Y, \tilde{X})\left(G(X)+\int \tilde{X} V\left(Y, Y^{\prime}\right)\left|\Psi\left(Y^{\prime}, \tilde{X}^{\prime}\right)\right|^{2}\right) \\
& \times\left(\tilde{X}-\int V\left(Y, Y^{\prime}\right)\left|\Psi\left(Y^{\prime}, \tilde{X}^{\prime}\right)\right|^{2}\right) \Psi(Y, \tilde{X}) \\
& +\int \Psi^{\dagger}(Y, \tilde{X})\left(\tilde{X}-\int V\left(Y, Y^{\prime}\right)\left|\Psi\left(Y^{\prime}, \tilde{X}^{\prime}\right)\right|^{2}\right)^{2} \Psi(Y, \tilde{X}) \\
= & \int \Psi^{\dagger}(Y, \tilde{X})\left(G(X)+\int V\left(Y, Y^{\prime}\right)\left|\Psi\left(Y^{\prime}, \tilde{X}^{\prime}\right)\right|^{2}\right)^{2} \Psi Y, \tilde{X} \\
& -2 \int \Psi^{\dagger}(Y, \tilde{X})\left(G(X)+\int V\left(Y, Y^{\prime}\right)\left|\Psi\left(Y^{\prime}, \tilde{X}^{\prime}\right)\right|^{2}\right) \\
& \times\left(\tilde{X}-\int \Psi^{\dagger}\left(Y^{\prime}, \tilde{X}^{\prime}\right) V\left(Y, Y^{\prime}\right) \Psi\left(Y^{\prime}, \tilde{X}^{\prime}\right)\right) \Psi(Y, \tilde{X})
\end{aligned}
$$

This last quantity is negligible since:

$$
\begin{aligned}
& \left|\int \Psi^{\dagger}(Y, \tilde{X})\left(G(X)+\int \tilde{X} V\left(Y, Y^{\prime}\right)\left|\Psi\left(Y^{\prime}, \tilde{X}^{\prime}\right)\right|^{2}\right)\left(\tilde{X}-\int\left|\Psi\left(Y^{\prime}, \tilde{X}^{\prime}\right)\right|^{2} \tilde{X} V\left(Y, Y^{\prime}\right)\right) \Psi(Y, \tilde{X})\right| \\
\leqslant & \left.\left\|\Psi^{\dagger}(Y, \tilde{X})\left(G(X)+\int \tilde{X} V\left(Y, Y^{\prime}\right)\left|\Psi\left(Y^{\prime}, \tilde{X}^{\prime}\right)\right|^{2}\right)\right\|\left\|\left(\tilde{X}-\int\left|\Psi\left(Y^{\prime}, \tilde{X}^{\prime}\right)\right|^{2} \tilde{X} V\left(Y, Y^{\prime}\right)\right) \Psi(Y, \tilde{X} 6)\right\|\right) \|
\end{aligned}
$$


and the norm of the last factor in (83) is close to zero, since:

$$
\begin{aligned}
& \left\|\left(\tilde{X}-\int \Psi^{\dagger}\left(Y^{\prime}, \tilde{X}^{\prime}\right) V\left(Y, Y^{\prime}\right) \Psi\left(Y^{\prime}, \tilde{X}^{\prime}\right)\right) \Psi(Y, \tilde{X})\right\|^{2} \\
= & \int \Psi^{\dagger}(Y, \tilde{X})\left(\tilde{X}-\int \Psi^{\dagger}\left(Y^{\prime}, \tilde{X}^{\prime}\right) V\left(Y, X^{\prime}\right) \Psi\left(Y^{\prime}, \tilde{X}^{\prime}\right)\right)^{2} \Psi(Y, \tilde{X}) \\
\rightarrow & 0
\end{aligned}
$$

Thus, the potential (60) simplifies as:

$$
\begin{aligned}
& \Psi^{\dagger}(Y, \tilde{X})\left(-\nabla_{\theta}\left(\vartheta^{2} \nabla_{\theta}-2\right)-\frac{1}{2} \nabla_{X}^{2}\right) \Psi(Y, \tilde{X}) \\
& +\int \Psi^{\dagger}(Y, \tilde{X})\left(G(X)+\int \Psi^{\dagger}\left(Y^{\prime}, \tilde{X}^{\prime}\right) V\left(Y, Y^{\prime}\right) \Psi\left(Y^{\prime}, \tilde{X}^{\prime}\right)\right)^{2} \Psi(Y, \tilde{X}) \\
= & \Psi^{\dagger}(Y)\left(-\frac{\sigma^{2}}{2} \nabla_{X}^{2}+\left(G(X)+\int \Psi^{\dagger}\left(Y^{\prime}\right) V\left(Y, Y^{\prime}\right) \Psi\left(Y^{\prime}\right)\right)^{2}\right) \Psi(Y)
\end{aligned}
$$

We can now come back to the initial variables, by letting $Y \rightarrow(X, \theta)$, and this yields:

$$
\begin{aligned}
& \Psi^{\dagger}(X, \theta)\left(-\nabla_{\theta}\left(\vartheta^{2} \nabla_{\theta}-2\right)-\frac{\sigma^{2}}{2} \nabla_{X}^{2}+\left(G(X)+\int V\left(X, X^{\prime}\right) \delta\left(\theta-\theta^{\prime}\right)\left|\Psi\left(X^{\prime}, \theta^{\prime}\right)\right|^{2}\right)^{2}\right) \Psi(X, \theta) \\
= & \Psi^{\dagger}(X, \theta)\left(-\nabla_{\theta}\left(\vartheta^{2} \nabla_{\theta}-2\right)-\frac{\sigma^{2}}{2} \nabla_{X}^{2}+\left(G(X)+\int V\left(X, X^{\prime}\right)\left|\Psi\left(X^{\prime}, \theta^{\prime}\right)\right|^{2}\right)^{2}\right) \Psi(X, \theta)
\end{aligned}
$$

We also change of variable for the dependence in the counting variable:

$$
\Psi(X, \theta)=\exp \left(\frac{\theta}{\vartheta^{2}}\right) \bar{\Psi}(X, \theta)
$$

and reset again:

$$
\bar{\Psi}(X, \theta) \rightarrow \Psi(X, \theta)
$$

Given our choice for $G$ and $V$ (57), and adding (58), the field theoretic version of (54), one obtains (55).

To close this section, we note that due to the change of variable (59) the source terms have to be included as they participate to the action. One thus has the action plus source terms:

$$
\begin{aligned}
& \Psi^{\dagger}(X, \tilde{X})\left(-\vartheta^{2} \nabla_{\theta}^{2}+\frac{1}{\vartheta^{2}}-\frac{1}{2} \nabla_{X}^{2}\right) \Psi(X, \tilde{X}) \\
& +\int \Psi^{\dagger}(X, \tilde{X})\left(G(X)+\int \Psi^{\dagger}\left(X^{\prime}, \tilde{X}^{\prime}\right) V\left(X, X^{\prime}\right) \Psi\left(X^{\prime}, \tilde{X}^{\prime}\right)\right)^{2} \Psi(X, \tilde{X}) \\
& +\int J(X) \exp \left(-\int(G(X)+\tilde{X})\right) \Psi(X, \tilde{X})+J^{\dagger}(X) \exp \left(\int(G(X)+\tilde{X})\right) \Psi^{\dagger}(X, \tilde{X}) \\
= & \Psi^{\dagger}(X)\left(-\vartheta^{2} \nabla_{\theta}^{2}+\frac{1}{\vartheta^{2}}-\frac{\sigma^{2}}{2} \nabla_{X}^{2}+\left(G(X)+\int \Psi^{\dagger}\left(X^{\prime}\right) V(X) \Psi\left(X^{\prime}\right)\right)^{2}\right) \Psi(X) \\
& +\int J(X) \exp \left(-\int(G(X)+\tilde{X})\right) \Psi(X)+J^{\dagger}(X) \exp \left(\int(G(X)+\tilde{X})\right) \Psi^{\dagger}(X)
\end{aligned}
$$

for an action including the source terms:

$$
\begin{aligned}
& \Psi^{\dagger}(X)\left(-\vartheta^{2} \nabla_{\theta}^{2}+\frac{1}{\vartheta^{2}}-\frac{1}{2} \nabla_{X}^{2}\right) \Psi(X)+\int \Psi^{\dagger}(X)\left(G(X)+\int \Psi^{\dagger}\left(X^{\prime}\right) V\left(X, X^{\prime}\right) \Psi\left(X^{\prime}\right)\right)^{2} \Psi(X) \\
& +\int J(X) \exp \left(-\int^{X} G(X)+X \tilde{X}(X)\right) \Psi(X)+\int J^{\dagger}(X) \exp \left(\int^{X} G(X)+X \tilde{X}(X)\right) \Psi^{\dagger}(X)
\end{aligned}
$$


The Green function are thus computed through the following formula:

$$
\begin{aligned}
G(X, Y)= & \left\langle\exp \left(-\left(\int^{X} G(X)+X \int \Psi^{\dagger}\left(X^{\prime}\right) V\left(X, X^{\prime}\right) \Psi\left(X^{\prime}\right)\right)\right)\right. \\
& \left.\times \Psi(X) \Psi^{\dagger}(Y) \exp \left(\left(\int^{Y} G(X)+Y \int \Psi^{\dagger}\left(X^{\prime}\right) V\left(Y, X^{\prime}\right) \Psi\left(X^{\prime}\right)\right)\right)\right\rangle \\
= & \left\langle\exp \left(-\left(\int_{Y}^{X} G(X)+\int \Psi^{\dagger}\left(X^{\prime}\right)\left(X V\left(X, X^{\prime}\right)-Y V\left(Y, X^{\prime}\right)\right) \Psi\left(X^{\prime}\right)\right)\right) \Psi(X) \Psi^{\dagger}(Y)\right\rangle
\end{aligned}
$$

\subsection{Appendix 2}

We inspect the case $\rho=0$.

As said in the text we look for a configuration that satisfies the market clearing condition. That is for $\bar{A}>>A$ we replace

$$
\Psi\left(K_{2}, P_{2}, X_{2}, \theta\right) \rightarrow \delta(P-H(K, X, \theta)) \Psi\left(K_{2}, X_{2}, \theta\right)
$$

As in the text we set $Z=(K, P, X, \theta)$. At zeroth order in $\frac{A}{A}$ the potential becomes:

$$
\left(\delta K-A P K^{\alpha}\left(1-\frac{\kappa}{d^{2}} \int \frac{P_{3} \exp \left(-\left(\frac{\left|X-X_{2}\right|}{d}+\frac{\left|X-X_{3}\right|}{d}\right)\right)}{P_{2}^{\gamma}}\left|\Psi\left(Z_{2}, \theta\right)\right|^{2}\left|\Psi\left(Z_{3}, \theta\right)\right|^{2}\right)\right)^{2}
$$

with constraint:

$$
P^{1+\gamma} K^{\alpha}=\frac{\kappa}{d^{2}} \int P_{2}\left(K_{2}\right)^{\alpha} P_{3} \exp \left(-\left(\frac{\left|X-X_{2}\right|}{d}+\frac{\left|X_{2}-X_{3}\right|}{d}\right)\right)\left|\Psi\left(Z_{2}, \theta\right)\right|^{2}\left|\Psi\left(Z_{3}, \theta\right)\right|^{2}
$$

The constraint is solved by looking for a field configuration (projection)

$$
\Psi(K, P, X, \theta)=\delta(P-H(K, X)) \Psi(K, X, \theta)
$$

for a function $P$

$$
P=H(K, X)=(K)^{-\frac{\alpha}{1+\gamma}} f(X)
$$

and replacing in the constraint, leads to:

$$
(f(X))^{1+\gamma}=\kappa \int P_{2}\left(K_{2}\right)^{\alpha} P_{3} \exp \left(-\left(\frac{\left|X-X_{2}\right|}{d}+\frac{\left|X_{2}-X_{3}\right|}{d}\right)\right)\left|\Psi\left(K_{2}, X_{2}, \theta\right)\right|^{2}\left|\Psi\left(K_{3}, X_{3}, \theta\right)\right|^{2}
$$

in average.

The potential for $K$ becomes:

$$
\begin{aligned}
& \left(\delta K-A(K)^{\frac{\alpha \gamma}{1+\gamma}} f(X)\left(1-\kappa \int \frac{\left(K_{3}\right)^{-\frac{\alpha}{1+\gamma}} f\left(X_{3}\right) \exp \left(-\left(\frac{\left|X-X_{2}\right|+\left|X-X_{3}\right|}{d}\right)\right)\left|\Psi\left(K_{2}, X_{2}, \theta\right)\right|^{2}\left|\Psi\left(K_{3}, X_{3}, \theta\right)\right|^{2}}{\left(\left(K_{2}\right)^{-\frac{\alpha}{1+\gamma}} f\left(X_{2}\right)\right)^{\gamma}}\right)^{2}\right. \\
= & \left(\delta K-A(K)^{\frac{\alpha \gamma}{1+\gamma}} f(X)\left(\begin{array}{c}
1-\kappa \int\left(K_{2}\right)^{\frac{\alpha \gamma}{1+\gamma}}\left(K_{3}\right)^{-\frac{\alpha}{1+\gamma}} f\left(X_{3}\right)\left(f\left(X_{2}\right)\right)^{-\gamma} \\
\times \exp \left(-\left(\frac{\left|X-X_{2}\right|}{d}+\frac{\left|X-X_{3}\right|}{d}\right)\right)\left|\Psi\left(K_{2}, X_{2}, \theta\right)\right|^{2}\left|\Psi\left(K_{3}, X_{3}, \theta\right)\right|^{2}
\end{array}\right)\right)^{2}
\end{aligned}
$$

for $\alpha \gamma<<1,(K)^{\frac{\alpha \gamma}{1+\gamma}} \simeq\langle K\rangle_{X}^{\frac{\alpha \gamma}{1+\gamma}}$, and this describes, for a given $X$, an harmonic oscillator of average $\langle K\rangle_{X}$ that satisfies:

$$
\delta\langle K\rangle_{X}-A\left(\langle K\rangle_{X}\right)^{\frac{\alpha \gamma}{1+\gamma}} f(X)\left(\begin{array}{c}
1-\kappa \int\left(K_{2}\right)^{\frac{\alpha \gamma}{1+\gamma}}\left(K_{3}\right)^{-\frac{\alpha}{1+\gamma}} f\left(X_{3}\right)\left(f\left(X_{2}\right)\right)^{-\gamma} \times \\
\times \exp \left(-\left(\frac{\left|X-X_{2}\right|}{d}+\frac{\left|X-X_{3}\right|}{d}\right)\right)\left|\Psi\left(K_{2}, X_{2}, \theta\right)\right|^{2}\left|\Psi\left(K_{3}, X_{3}, \theta\right)\right|^{2}
\end{array}\right)=0
$$


with solution:

$$
\langle K\rangle_{X}=\left(\frac{A}{\delta} f(X)\right)^{\frac{1+\gamma}{1+\gamma(1-\alpha)}}\left(\begin{array}{c}
1-\kappa \int\left(K_{2}\right)^{\frac{\alpha \gamma}{1+\gamma}}\left(K_{3}\right)^{-\frac{\alpha}{1+\gamma}} f\left(X_{3}\right)\left(f\left(X_{2}\right)\right)^{-\gamma} \times \\
\times \exp \left(-\left(\frac{\left|X-X_{2}\right|}{d}+\frac{\left|X-X_{3}\right|}{d}\right)\right)\left|\Psi\left(K_{2}, X_{2}, \theta\right)\right|^{2}\left|\Psi\left(K_{3}, X_{3}, \theta\right)\right|^{2}
\end{array}\right)^{\frac{1+\gamma}{1+\gamma(1-\alpha)}}
$$

This expression can thus be used in (??), to identify $f(X)$ :

$$
\begin{aligned}
(f(X))^{1+\gamma}= & \kappa \int\left(K_{2}\right)^{\frac{\alpha \gamma}{1+\gamma}}\left(K_{3}\right)^{-\frac{\alpha}{1+\gamma}} f\left(X_{3}\right) f\left(X_{2}\right) \\
& \exp \left(-\left(\frac{\left|X-X_{2}\right|}{d}+\frac{\left|X_{2}-X_{3}\right|}{d}\right)\right)\left|\Psi\left(K_{2}, X_{2}, \theta\right)\right|^{2}\left|\Psi\left(K_{3}, X_{3}, \theta\right)\right|^{2}
\end{aligned}
$$

At the lowest order of perturbation theory (65) becomes:

$$
\begin{aligned}
(f(X))^{1+\gamma} \simeq & \kappa \int G\left(\left(K_{3}, X_{3}, \theta\right),\left(K_{2}, X_{2}, \theta\right)\right) G\left(\left(K_{2}, X_{2}, \theta\right),\left(K_{3}, X_{3}, \theta\right)\right)\left(K_{2}\right)^{\frac{\alpha \gamma}{1+\gamma}}\left(K_{3}\right)^{-\frac{\alpha}{1+\gamma}} \\
& \times f\left(X_{3}\right) f\left(X_{2}\right) \exp \left(-\left(\frac{\left|X-X_{2}\right|}{d}+\frac{\left|X_{2}-X_{3}\right|}{d}\right)\right)
\end{aligned}
$$

In Appendix 3, we will derive the Green functions. It appears that for $\theta^{\prime} \simeq \theta$, The Green functions are those of harmonic oscillators for a propagation time of order $\vartheta^{2}$ that we will write $G\left(\left(K_{3}, X_{3}\right),\left(K_{2}, X_{2}\right)\right)$. We thus have:

$$
(f(X))^{1+\gamma} \simeq \kappa \int G\left(\left(K_{3}, X_{2}\right),\left(K_{2}, X_{2}\right)\right) G\left(\left(K_{2}, X_{2}\right),\left(K_{3}, X_{3}\right)\right)\left(K_{2}\right)^{\frac{\alpha \gamma}{1+\gamma}}\left(K_{3}\right)^{-\frac{\alpha}{1+\gamma}}\left(f\left(X_{2}\right)\right)^{2} \exp \left(-\frac{\left|X-X_{2}\right|}{d}\right)
$$

We also assume that the dynamic for $K$ is "faster" than the dynamics for $X$, so that the Green functions have the form:

$$
G\left(\left(K_{3}, X_{3}\right),\left(K_{2}, X_{2}\right)\right)=G_{X_{2}}\left(K_{3}, K_{2}\right) G\left(X_{2}, X_{2}\right)
$$

As a consequence, the integrals over $K_{2}$ and $K_{3}$ :

$$
\int G_{X_{2}}\left(K_{3}, K_{2}\right)\left(K_{2}\right)^{\frac{\alpha \gamma}{1+\gamma}}\left(K_{3}\right)^{-\frac{\alpha}{1+\gamma}} G_{X_{3}}\left(K_{3}, K_{2}\right) d K_{2} d K_{3}
$$

compute the average of $\left(K_{2}\right)^{\frac{\alpha \gamma}{1+\gamma}}\left(K_{3}\right)^{-\frac{\alpha}{1+\gamma}}$ for a transition from state $X_{2}$ to state $X_{3}$. As a consequence:

$$
\begin{aligned}
& \int G\left(\left(K_{3}, X_{3}\right),\left(K_{2}, X_{2}\right)\right)\left(K_{2}\right)^{\frac{\alpha \gamma}{1+\gamma}}\left(K_{3}\right)^{-\frac{\alpha}{1+\gamma}} G\left(\left(K_{2}, X_{2}\right),\left(K_{3}, X_{3}\right)\right) d K_{2} d K_{3} \\
\simeq & G\left(X_{2}, X_{3}\right) G\left(X_{3}, X_{2}\right)\langle K\rangle_{X_{2}}^{\frac{\alpha \gamma}{1+\gamma}}\langle K\rangle_{X_{3}}^{-\frac{\alpha}{1+\gamma}}
\end{aligned}
$$

where $\langle K\rangle_{X_{2}}$ is the average level of capital for agent with $X=X_{2}$ is computed below by identification.

As a consequence:

$$
(f(X))^{1+\gamma} \simeq \frac{\kappa}{d^{2}} \int G\left(X_{2}, X_{3}\right) G\left(X_{3}, X_{2}\right)\langle K\rangle_{X_{2}}^{\frac{\alpha \gamma}{1+\gamma}}\langle K\rangle_{X_{3}}^{-\frac{\alpha}{1+\gamma}} f\left(X_{3}\right) f\left(X_{2}\right) \exp \left(-\left(\frac{\left|X-X_{2}\right|}{d}+\frac{\left|X_{2}-X_{3}\right|}{d}\right)\right)
$$

The potential for $X$ is harmonic in zeroth order in $\kappa$ with low frequency and then we can assume that the integral is distributed around $X_{2}-X_{3} \simeq 0$ and replace $\exp \left(-\left(\frac{\left|X_{2}-X_{3}\right|}{d}\right)\right)$ by $d$ times a delta function. As a consequence:

$$
(f(X))^{1+\gamma} \simeq \frac{\kappa}{d} \int\langle K\rangle_{X_{2}}^{\frac{\alpha(\gamma-1)}{1+\gamma}}\left(f\left(X_{2}\right)\right)^{2} G\left(X_{2}, X_{2}\right) \exp \left(-\left(\frac{\left|X-X_{2}\right|}{d}\right)\right)
$$

If the exchange positions are homogenously spread on $[-1,1]$, one can replace the probability $G\left(X_{2}, X_{2}\right)$ by a constant density, here $\frac{1}{2}$ in first approximation, to normalize the probability of the interval $[-1,1]$ to 1 . 


$$
(f(X))^{1+\gamma} \simeq \frac{\kappa}{2 d} \int\langle K\rangle_{X_{2}}^{\frac{\alpha(\gamma-1)}{1+\gamma}}\left(f\left(X_{2}\right)\right)^{2} \exp \left(-\frac{\left|X-X_{2}\right|}{d}\right)
$$

Thus, using (64) one can express $\langle K\rangle_{X}$ as a function of $X$ :

$$
\begin{aligned}
\langle K\rangle_{X} & =\left(\frac{A}{\delta} f(X)\right)^{\frac{1+\gamma}{1+\gamma(1-\alpha)}}\left(\begin{array}{c}
1-\frac{\kappa}{4 d^{2}} \int\left(K_{2}\right)^{\frac{\alpha \gamma}{1+\gamma}}\left(K_{3}\right)^{-\frac{\alpha}{1+\gamma}} f\left(X_{3}\right)\left(f\left(X_{2}\right)\right)^{-\gamma} \times \\
\times \exp \left(-\left(\frac{\left|X-X_{2}\right|}{d}+\frac{\left|X-X_{3}\right|}{d}\right)\right)\left|\Psi\left(K_{2}, X_{2}, \theta\right)\right|^{2}\left|\Psi\left(K_{3}, X_{3}, \theta\right)\right|^{2}
\end{array}\right)^{\frac{1+\gamma}{1+\gamma(1-\alpha)}} \\
& \simeq\left(\frac{A}{\delta} f(X)\right)^{\frac{1+\gamma}{1+\gamma(1-\alpha)}}\left(1-\frac{\kappa}{4 d^{2}} \int\langle K\rangle_{X_{2}}^{\frac{\alpha \gamma}{1+\gamma}}\langle K\rangle_{X_{3}}^{-\frac{\alpha}{1+\gamma}} f\left(X_{3}\right)\left(f\left(X_{2}\right)\right)^{-\gamma} \exp \left(-\left(\frac{\left|X-X_{2}\right|}{d}+\frac{\left|X-X_{3}\right|}{d}\right)\right)\right)
\end{aligned}
$$

for $\gamma<<1$. The factor $\frac{1}{4}$ appears again to normalize the probability each interval $[-1,1]$ to 1 . We thus postulate the following form for $\langle K\rangle_{X}$ :

$$
\langle K\rangle_{X}=\left(\frac{A}{\delta} f(X)\left(1-h \exp \left(-\frac{|X|}{d}\right)\left(1-\frac{\cosh \frac{X}{d}}{\exp \left(\frac{1}{d}\right)}\right)\right)\right)^{\frac{1+\gamma}{1+\gamma(1-\alpha)}}
$$

with $h$ to be determined. In (66), we can replace for the sake of simplicity, $1-h \exp \left(-\frac{|X|}{d}\right)\left(1-\frac{\cosh \frac{X}{d}}{\exp \left(\frac{1}{d}\right)}\right)$ by its average which is approximatively equal to $1-h \exp \left(-\frac{1}{2 d}\right)\left(1-\exp \left(-\frac{1}{2 d}\right)\right),\left(h<\exp \left(\frac{1}{2 d}\right)\right)$, so that (66) rewrites:

$$
\begin{aligned}
&\langle K\rangle_{X} \simeq\left(\frac{A}{\delta} f(X)\right)^{\frac{1+\gamma}{1+\gamma(1-\alpha)}}\left(\begin{array}{c}
1-\frac{\kappa}{4 d d^{2}} \int\left(\frac{A}{\delta} f\left(X_{2}\right)\left(1-h \exp \left(-\frac{1}{2 d}\right)\left(1-\exp \left(-\frac{1}{2 d}\right)\right)\right)\right)^{\frac{\alpha \gamma}{1+\gamma(1-\alpha)}} \\
\times\left(\frac{A}{\delta} f\left(X_{3}\right)(1-h)\right)^{-\frac{\alpha}{1+\gamma(1-\alpha)}} f\left(X_{3}\right)\left(f\left(X_{2}\right)\right)^{-\gamma} \exp \left(-\left(\frac{\left|X-X_{2}\right|}{d}+\frac{\left|X-X_{3}\right|}{d}\right)\right)
\end{array}\right) \\
& \simeq\left(\frac{A}{\delta} f(X)\right)^{\frac{1+\gamma}{1+\gamma(1-\alpha)}}\left(\begin{array}{c}
1-\frac{\kappa}{4 d^{2}}\left(\frac{A}{\delta}\left(1-h \exp \left(-\frac{1}{2 d}\right)\left(1-\exp \left(-\frac{1}{2 d}\right)\right)\right)\right)^{-\frac{\alpha(1-\gamma)}{1+\gamma(1-\alpha)}} \\
\times \int\left(f\left(X_{3}\right)\right)^{\frac{(1-\alpha)(1+\gamma)}{1+\gamma(1-\alpha)}}\left(f\left(X_{2}\right)\right)^{-\gamma \frac{1-\alpha(1-\gamma)}{1+\gamma(1-\alpha)}} \exp \left(-\left(\frac{\left|X-X_{2}\right|}{d}+\frac{\left|X-X_{3}\right|}{d}\right)\right)
\end{array}\right)
\end{aligned}
$$

and the equation for $f(X)$ becomes:

$$
\begin{aligned}
(f(X))^{1+\gamma} \simeq & \frac{\kappa}{2 d} \int\langle K\rangle_{X_{2}}^{\frac{\alpha(\gamma-1)}{1+\gamma}}\left(f\left(X_{2}\right)\right)^{2} \exp \left(-\frac{\left|X-X_{2}\right|}{d}\right) \\
\simeq & \frac{\kappa}{2 d}\left(\frac{A}{\delta}\right)^{\frac{\alpha(\gamma-1)}{1+\gamma(1-\alpha)}} \int\left(1-h \exp \left(-\frac{|X|}{d}\right)\left(1-\frac{\cosh \left(\frac{X}{d}\right)}{\exp \left(\frac{1}{d}\right)}\right)\right)^{\frac{\alpha(\gamma-1)}{1+\gamma(1-\alpha)}} \\
& \times\left(f\left(X_{2}\right)\right)^{2+\frac{\alpha(\gamma-1)}{1+\gamma(1-\alpha)}} \exp \left(-\frac{\left|X-X_{2}\right|}{d}\right)
\end{aligned}
$$

Again, we can replace for the sake of simplicity, $1-h \exp \left(-\frac{|X|}{d}\right)\left(1-\frac{\cosh \left(\frac{x}{d}\right)}{\exp \left(\frac{1}{d}\right)}\right)$ by its average $\simeq 1-$ $h \exp \left(-\frac{1}{2 d}\right)\left(1-\frac{\exp \left(-\frac{1}{2 d}\right)}{2}\right),\left(h<\exp \left(\frac{1}{2 d}\right)\right)$. We look for a solution:

$$
f(X)=D \exp \left(-c \frac{|X|}{d}\right)
$$

and the identification becomes for $\gamma<<1$ :

$$
\begin{aligned}
\exp \left(-(1+\gamma) c \frac{|X|}{d}\right) \simeq & \frac{\kappa}{2 d}\left(\frac{A}{\delta}\right)^{\frac{\alpha(\gamma-1)}{1+\gamma(1-\alpha)}} D^{\frac{\left(1-\gamma^{2}\right)(1-\alpha)}{1+\gamma(1-\alpha)}}\left(\left(1-h \exp \left(-\frac{1}{2 d}\right)\left(1-\frac{\exp \left(-\frac{1}{2 d}\right)}{2}\right)\right)\right)^{\frac{\alpha(\gamma-1)}{1+\gamma(1-\alpha)}} \\
& \times \int \exp \left(-c \frac{(1+\gamma)(2-\alpha)}{1+\gamma(1-\alpha)} \frac{\left|X_{2}\right|}{d}\right) \exp \left(-\frac{\left|X-X_{2}\right|}{d}\right)
\end{aligned}
$$


The integral can be estimated as $(X>0)$ :

$$
\begin{aligned}
& \int_{-1}^{0} \exp \left(\frac{2 a X_{2}}{d}\right) \exp \left(-\frac{\left(X-X_{2}\right)}{d}\right)+\int_{0}^{X} \exp \left(-\frac{2 a X_{2}}{d}\right) \exp \left(-\frac{\left(X-X_{2}\right)}{d}\right) \\
& +\int_{X}^{1} \exp \left(-\frac{2 a X_{2}}{d}\right) \exp \left(\frac{\left(X-X_{2}\right)}{d}\right) \\
= & d\left(\frac{\exp \left(-\frac{X}{d}\right)\left(1-\exp \left(-\frac{(2 a+1)}{d}\right)\right)}{2 a+1}+\left(\frac{\exp \left(-\frac{2 a X}{d}\right)-\exp \left(-\frac{X}{d}\right)}{1-2 a}\right)+\frac{\exp \left(-\frac{2 a X}{d}\right)-\exp \left(\frac{X-(2 a+1)}{d}\right)}{2 a+1}\right) \\
\simeq & d \frac{4 a \exp \left(-\frac{X}{d}\right)-2 \exp \left(-\frac{2 a X}{d}\right)}{4 a^{2}-1}
\end{aligned}
$$

and the identification for $f(X)$ becomes:

$$
\begin{aligned}
\exp \left(-(1+\gamma) c \frac{|X|}{d}\right)= & \frac{\kappa}{2}\left(\frac{A}{\delta}\right)^{\frac{\alpha(\gamma-1)}{1+\gamma(1-\alpha)}} D^{\frac{\left(1-\gamma^{2}\right)(1-\alpha)}{1+\gamma(1-\alpha)}}\left(\left(1-h \exp \left(-\frac{1}{2 d}\right)\left(1-\exp \left(-\frac{1}{2 d}\right)\right)\right)\right)^{\frac{\alpha(\gamma-1)}{1+\gamma(1-\alpha)}} \\
& \times\left(\frac{4 a \exp \left(-\frac{X}{d}\right)-2 \exp \left(-2 a \frac{X}{d}\right)}{4 a^{2}-1}\right)
\end{aligned}
$$

For $\gamma<1$, one has:

$$
\begin{aligned}
c & \simeq \frac{1}{1+\gamma} \\
2 a & =\frac{(1+\gamma)(2-\alpha)}{1+\gamma(1-\alpha)} \frac{1}{1+\gamma} \\
& =\frac{2-\alpha}{1+\gamma(1-\alpha)}>1
\end{aligned}
$$

That yields the identification for $D$ :

$$
\frac{\kappa}{2}\left(\frac{A}{\delta}\right)^{\frac{\alpha(\gamma-1)}{1+\gamma(1-\alpha)}} D^{\frac{\left(1-\gamma^{2}\right)(1-\alpha)}{1+\gamma(1-\alpha)}} \frac{\frac{2(2-\alpha)}{1+\gamma(1-\alpha)}}{\left(\frac{2-\alpha}{1+\gamma(1-\alpha)}\right)^{2}-1}\left(\left(1-h \exp \left(-\frac{1}{2 d}\right)\left(1-\frac{\exp \left(-\frac{1}{2 d}\right)}{2}\right)\right)\right)^{\frac{\alpha(\gamma-1)}{1+\gamma(1-\alpha)}}=1
$$

whose solution is:

$D \simeq\left(\frac{1+\gamma(1-\alpha)}{(2-\alpha) \kappa}\left(\left(\frac{2-\alpha}{1+\gamma(1-\alpha)}\right)^{2}-1\right)\left(\frac{A}{\delta}\left(1-h \exp \left(-\frac{1}{2 d}\right)\left(1-\frac{\exp \left(-\frac{1}{2 d}\right)}{2}\right)\right)\right)^{\frac{\alpha(1-\gamma)}{1+\gamma(1-\alpha)}}\right)^{\frac{1+\gamma(1-\alpha)}{\left(1-\gamma^{2}\right)(1-\alpha)}}$

and we are lead ultimately to:

$$
\begin{aligned}
f(X)= & D \exp \left(-\frac{|X|}{(1+\gamma) d}\right) \\
= & \left(\frac{A}{\delta}\left(1-h \exp \left(-\frac{1}{2 d}\right)\left(1-\frac{\exp \left(-\frac{1}{2 d}\right)}{2}\right)\right)\right)^{\frac{\alpha}{(1-\alpha)(\gamma+1)}} \\
& \times\left(\frac{1+\gamma(1-\alpha)}{(2-\alpha) \kappa}\left(\left(\frac{2-\alpha}{1+\gamma(1-\alpha)}\right)^{2}-1\right)\right)^{\frac{1+\gamma(1-\alpha)}{\left(1-\gamma^{2}\right)(1-\alpha)}} \exp \left(-\frac{|X|}{d(1+\gamma)}\right)
\end{aligned}
$$


and using (67):

$$
\begin{aligned}
\langle K\rangle_{X}= & \left(\frac{1+\gamma(1-\alpha)}{(2-\alpha) \kappa}\left(\left(\frac{2-\alpha}{1+\gamma(1-\alpha)}\right)^{2}-1\right)\right)^{\frac{1}{(1-\gamma)(1-\alpha)}} \\
& \times\left(\left(1-h \exp \left(-\frac{1}{2 d}\right)\left(1-\frac{\exp \left(-\frac{1}{2 d}\right)}{2}\right)\right)\right)^{\frac{\alpha}{(1-\alpha)(1+\gamma(1-\alpha))}}\left(\frac{A}{\delta}\right)^{-\frac{1}{(1-\alpha)}} \\
& \times \exp \left(-\frac{|X|}{(1+\gamma(1-\alpha)) d}\right)\left(\left(1-h \exp \left(-\frac{|X|}{d}\right)\left(1-\frac{\cosh \left(\frac{X}{d}\right)}{\exp \left(\frac{1}{d}\right)}\right)\right)\right)^{\frac{1+\gamma}{1+\gamma(1-\alpha)}}
\end{aligned}
$$

Then we can use the condition (66) on $\langle K\rangle_{X}$ and compute $g$ and $h$ (we consider $\gamma<<1$ ):

$$
\begin{aligned}
U= & 1-\frac{\kappa}{4 d^{2}}\left(\frac{A}{\delta}\left(1-h \exp \left(-\frac{1}{2 d}\right)\left(1-\frac{\exp \left(-\frac{1}{2 d}\right)}{2}\right)\right)\right)^{-\frac{\alpha(1-\gamma)}{1+\gamma(1-\alpha)}} \int\left(f\left(X_{3}\right)\right)^{\frac{(1-\alpha)(1+\gamma)}{1+\gamma(1-\alpha)}}\left(f\left(X_{2}\right)\right)^{-\gamma \frac{(1-\alpha)(1+\gamma)}{1+\gamma(1-\alpha)}} \\
& \times \exp \left(-\left(\frac{\left|X-X_{2}\right|}{d}+\frac{\left|X-X_{3}\right|}{d}\right)\right) \\
\simeq & 1-\frac{1}{4 d^{2}}\left(\frac{1+\gamma(1-\alpha)}{(2-\alpha)}\left(\left(\frac{2-\alpha}{1+\gamma(1-\alpha)}\right)^{2}-1\right)\right) \\
& \times \int \exp \left(-\frac{(1-\alpha)}{1+\gamma(1-\alpha)} \frac{\left|X_{3}\right|}{d}\right) \exp \left(-\gamma \frac{(1-\alpha)}{1+\gamma(1-\alpha)} \frac{\left|X_{2}\right|}{d}\right) \exp \left(-\left(\frac{\left|X-X_{2}\right|}{d}+\frac{\left|X-X_{3}\right|}{d}\right)\right) \\
\simeq & 1-\frac{1}{4 d^{2}}\left(\frac{1+\gamma(1-\alpha)}{(2-\alpha)}\left(\left(\frac{2-\alpha}{1+\gamma(1-\alpha)}\right)^{2}-1\right)\right) \\
& \times \int \exp \left(-\frac{(1-\alpha)}{1+\gamma(1-\alpha)} \frac{\left|X_{3}\right|}{d}\right) \exp \left(-\left(\frac{\left|X-X_{2}\right|}{d}+\frac{\left|X-X_{3}\right|}{d}\right)\right) \\
\simeq & 1-\frac{1}{2 d^{2}} \frac{1+\gamma(1-\alpha)}{(2-\alpha)}\left(\left(\frac{2-\alpha}{1+\gamma(1-\alpha)}\right)^{2}-1\right) \int \exp \left(-\frac{(1-\alpha)}{1+\gamma(1-\alpha)} \frac{\left|X_{3}\right|}{d}\right) \exp \left(-\frac{\left|X-X_{3}\right|}{d}\right) \\
= & 1-\frac{1}{2 d} \frac{1+\gamma(1-\alpha)}{(2-\alpha)}\left(\left(\frac{2-\alpha}{1+\gamma(1-\alpha)}\right)^{2}-1\right) \int \exp \left(-\frac{(1-\alpha)}{1+\gamma(1-\alpha)} \frac{\left|X_{3}\right|}{d}\right)\left(1-\frac{\cosh \left(\frac{X}{d}\right)}{\exp \left(\frac{1}{d}\right)}\right)
\end{aligned}
$$

since:

$$
\int \exp \left(-\left(\frac{\left|X-X_{2}\right|}{d}\right)\right)=2 d\left(1-\frac{\cosh \left(\frac{X}{d}\right)}{\exp \left(\frac{1}{d}\right)}\right)
$$

Then $g$ and $h$ will be found by computing the following integral (for $X>0$, the general case being obtained by replacing $X$ with $|X|$ :

$$
\begin{aligned}
& \int \exp \left(-\frac{(1-\alpha)}{1+\gamma(1-\alpha)} \frac{\left|X_{3}\right|}{d}\right) \exp \left(-\frac{\left|X-X_{3}\right|}{d}\right) \\
\simeq & \int \exp \left(-(1-\alpha) \frac{\left|X_{3}\right|}{d}\right) \exp \left(-\frac{\left|X-X_{3}\right|}{d}\right) d X_{3} \\
& \int_{-1}^{0} \exp \left((1-\alpha) \frac{u}{d}-\frac{X-u}{d}\right) d u+\int_{X}^{1} \exp \left(-(1-\alpha) \frac{u}{d}+\frac{X-u}{d}\right) d u \\
& +\int_{0}^{X} \exp \left(-(1-\alpha) \frac{u}{d}-\frac{X-u}{d}\right) d u \\
= & d\left(\frac{e^{\alpha \frac{X}{d}}-1}{\alpha} e^{-\frac{X}{d}}+\frac{e^{\frac{X+\alpha-2}{d}}-e^{\frac{X}{d}(\alpha-1)}}{\alpha-2}-e^{-\frac{X}{d}} \frac{1-e^{\frac{\alpha-2}{d}}}{\alpha-2}\right)
\end{aligned}
$$


which can be approximated at the zeroth order in $\alpha$ :

$$
\begin{aligned}
& \simeq d\left(\frac{1}{2} e^{-\frac{X}{d}}-\frac{1}{2} e^{\frac{X-2}{d}}-e^{-\frac{X}{d}}\left(\frac{1}{2} e^{-\frac{2}{d}}-\frac{1}{2}\right)+X e^{-\frac{X}{d}}\right) \\
& \simeq d\left(\frac{X}{d}+1-\frac{1}{2} e^{\frac{2 X-2}{d}}\right) e^{-\frac{X}{d}}
\end{aligned}
$$

The correction terms of order $\alpha$ are negligible in the neighborhood of $d=1$. In average, the previous formula is equal to $d\left(\frac{3}{2 d}-\frac{1}{2} e^{-1}\right) e^{-\frac{X}{d}}$. Restoring the absolute value, one finds for $d$ close to 1 :

$$
h \simeq \frac{1+\gamma(1-\alpha)}{2(1-\alpha)}\left(\frac{1+\gamma(1-\alpha)}{(2-\alpha)}\left(\left(\frac{2-\alpha}{1+\gamma(1-\alpha)}\right)^{2}-1\right)\right)\left(\frac{3}{2}-\frac{e^{-\frac{1}{d}}}{2}\right)
$$

As a consequence of the previous computations, the potential becomes:

$$
\delta^{2}\left(K-\langle K\rangle_{X}\right)^{2}
$$

which justifies the assumption of harmonic oscillations.

The price can be rewritten as a function of $\langle K\rangle_{X}$ using (67):

$$
\begin{aligned}
P= & H(K, X)=(K)^{-\frac{\alpha}{1+\gamma}} f(X) \\
& \left(\frac{K}{\langle K\rangle_{X}}\right)^{-\frac{\alpha}{1+\gamma}} \frac{f(X)}{\left(\frac{A}{\delta} f(X)\left(1-h \exp \left(-\frac{|X|}{d}\right)\left(1-\frac{\cosh \frac{X}{d}}{\exp \left(\frac{1}{d}\right)}\right)\right)\right)^{\frac{\alpha}{1+\gamma(1-\alpha)}}} \\
= & \left(\frac{K}{\langle K\rangle_{X}}\right)^{-\frac{\alpha}{1+\gamma}} \frac{(f(X))^{\frac{(1+\gamma)(1-\alpha)}{1+\gamma(1-\alpha)}}}{\left(\frac{A}{\delta}\left(1-h \exp \left(-\frac{|X|}{d}\right)\left(1-\frac{\cosh \frac{X}{d}}{\exp \left(\frac{d}{d}\right)}\right)\right)\right)^{\frac{\alpha}{1+\gamma(1-\alpha)}}}
\end{aligned}
$$

and thus, 67):

$$
P=\frac{\left(\frac{1+\gamma(1-\alpha)}{(2-\alpha) \kappa}\left(\left(\frac{2-\alpha}{1+\gamma(1-\alpha)}\right)^{2}-1\right)\right)^{\frac{1}{1-\gamma}} \exp \left(-\frac{(1-\alpha)|X|}{d(1+\gamma(1-\alpha))}\right)}{\left(\frac{K}{\langle K\rangle_{X}}\right)^{\frac{\alpha}{1+\gamma}}\left(\left(\frac{1-h \exp \left(-\frac{|X|}{d}\right)\left(1-\frac{\cosh \frac{X}{d}}{\exp \left(\frac{1}{d}\right)}\right)}{1-h \exp \left(-\frac{1}{2 d}\right)\left(1-\frac{\exp \left(-\frac{1}{2 d}\right)}{2}\right)}\right)\right)^{\frac{\alpha}{1+\gamma(1-\alpha)}}}
$$

Having found the price for $\bar{A} \rightarrow \infty$, we can now consider the corrections due to $A$ :

$\bar{A}>>A$ : once again we consider fields of the form: $\Psi(K, P, X, \theta) \rightarrow \delta(P-F(K, X, \theta)) \Psi(K, X, \theta)$.

The potential considered is now:

$$
\begin{aligned}
& \left(\delta K-A P K^{\alpha}\left(1-\kappa \int \frac{P_{3} \exp \left(-\left(\left|X_{1}-X_{2}\right|+\left|X_{1}-X_{3}\right|\right)\right)}{P_{2}^{\gamma}}\left|\Psi\left(K_{2}, X_{2}, \theta\right)\right|^{2}\left|\Psi\left(K_{3}, X_{3}, \theta\right)\right|^{2}\right)\right)^{2} \\
+ & \bar{A}^{2}\left(P^{1+\gamma} K^{\alpha}-\kappa \int P_{2}\left(K_{2}\right)^{\alpha} P_{3} \exp \left(-\left(\left|X_{1}-X_{2}\right|+\left|X_{2}-X_{3}\right|\right)\right)\left|\Psi\left(K_{2}, X_{2}, \theta\right)\right|^{2}\left|\Psi\left(K_{3}, X_{3}, \theta\right)\right|^{2}\right)^{2} \\
= & \left(\delta K-A P K^{\alpha} U\right)^{2}+\bar{A}^{2}\left(P^{1+\gamma} K^{\alpha}-V\right)^{2}
\end{aligned}
$$

with:

$$
\begin{aligned}
U & =\left(1-\kappa \int \frac{P_{3} \exp \left(-\left(\left|X_{1}-X_{2}\right|+\left|X_{1}-X_{3}\right|\right)\right)}{P_{2}^{\gamma}}\left|\Psi\left(K_{2}, X_{2}, \theta\right)\right|^{2}\left|\Psi\left(K_{3}, X_{3}, \theta\right)\right|^{2}\right) \\
V & =\kappa \int P_{2}\left(K_{2}\right)^{\alpha} P_{3} \exp \left(-\left(\left|X_{1}-X_{2}\right|+\left|X_{2}-X_{3}\right|\right)\right)\left|\Psi\left(K_{2}, X_{2}, \theta\right)\right|^{2}\left|\Psi\left(K_{3}, X_{3}, \theta\right)\right|^{2}
\end{aligned}
$$


The price is found through the minimization of the sum:

$$
\left(\delta K-A P K^{\alpha} U\right)^{2}+\bar{A}^{2}\left(P^{1+\gamma} K^{\alpha}-V\right)^{2}
$$

That yields the condition:

$$
-U A K^{\alpha}\left(\delta K-A P K^{\alpha} U\right)+(1+\gamma) P^{\gamma} \bar{A}^{2} K^{\alpha}\left(P^{1+\gamma} K^{\alpha}-V\right)=0
$$

A first order approximation in $\gamma$ of (70) is:

$$
-A K^{\alpha}\left(\delta K-A P K^{\alpha}\right)+(1+\gamma) P^{\gamma} \bar{A}^{2} K^{\alpha}\left(P^{1+\gamma} K^{\alpha}-V\right) \simeq 0
$$

We we then use (71) at first order in $\gamma$ and in $\frac{A^{2}}{A^{2}}$ to compute $P^{1+\gamma} K^{\alpha}$ :

$$
\begin{aligned}
P^{1+\gamma} K^{\alpha} & =V+\frac{A\left(\delta K-A P K^{\alpha}\right)}{\bar{A}^{2}(1+\gamma) P^{\gamma}} \simeq V+\frac{A\left(\delta K-A P K^{\alpha}\right)}{\bar{A}^{2}} \\
& \simeq V+\frac{A K^{\alpha}(\delta K-A V)}{\bar{A}^{2} K^{\alpha}}
\end{aligned}
$$

Now, we compute $P^{1+\gamma} K^{\alpha}$ at first order in $\frac{A^{2}}{A^{2}}$ :

$$
\begin{aligned}
P^{1+\gamma} K^{\alpha}-A \frac{\delta K-A V}{A^{2}+\bar{A}^{2}} & =V \\
P^{1+\gamma} K^{\alpha}-\frac{\delta A K}{A^{2}+\bar{A}^{2}} & =\frac{\bar{A}^{2} V}{A^{2}+\bar{A}^{2}} \\
P^{1+\gamma} K^{\alpha} & =V\left(1+A \frac{\delta K-A V}{V\left(A^{2}+\bar{A}^{2}\right)}\right)
\end{aligned}
$$

For this value, the potentials become:

$$
\begin{aligned}
& \left(\delta K-A P K^{\alpha} U\right)^{2}+\bar{A}^{2}\left(P^{1+\gamma} K^{\alpha}-V\right)^{2} \\
\simeq & \left(\delta K-A P K^{\alpha} U\right)^{2}+\bar{A}^{2} A^{2}\left(\frac{\delta K-A U V}{\left(A^{2} U^{2}+\bar{A}^{2}\right)}\right)^{2} \\
= & \delta^{2}\left(1+\frac{\bar{A}^{2} A^{2}}{\left(A^{2} U^{2}+\bar{A}^{2}\right)^{2}}\right)\left(K-\frac{A P K^{\alpha} U+\frac{\bar{A}^{2} A^{2}}{\left(A^{2} U^{2}+\bar{A}^{2}\right)^{2}} A U V}{\delta\left(1+\frac{\bar{A}^{2} A^{2}}{\left(A^{2} U^{2}+\bar{A}^{2}\right)^{2}}\right)}\right)^{2} \\
+ & \frac{\bar{A}^{2} A^{2}\left(A P K^{\alpha} U-A U V\right)^{2}}{\bar{A}^{2} A^{2}+\left(A^{2} U^{2}+\bar{A}^{2}\right)^{2}}
\end{aligned}
$$

Equations (72) and (73) can be simplified. Since we are looking for first order corrections in $\frac{A^{2}}{A^{2}}$, in (72) we can replace we can replace $\delta K-A V$ by its lowest order approximation, that is, given that we have assumed $\gamma<<1$ :

$$
\delta K-A V=\delta K-A P^{1+\gamma} K^{\alpha} \simeq \delta K-A P K^{\alpha}
$$

To this order of approximation, one can also replace $K$ by $\langle K\rangle_{X}$ and using (63):

$$
\delta\langle K\rangle_{X}=A P\langle K\rangle_{X}^{\alpha} U
$$

and (67):

$$
\begin{aligned}
\frac{\delta K-A V}{V} & \simeq A \frac{\delta\langle K\rangle_{X}-A P\langle K\rangle_{X}^{\alpha}}{A P\langle K\rangle_{X}^{\alpha}} \\
& \simeq A \frac{\delta\langle K\rangle_{X}-A P\langle K\rangle_{X}^{\alpha}}{A P\langle K\rangle_{X}^{\alpha}} \\
& =A(U-1) \simeq A(\langle U\rangle-1) \simeq-h A\left(1-\exp \left(-\frac{1}{d}\right)\right)
\end{aligned}
$$


and (72) becomes:

$$
P^{1+\gamma} K^{\alpha}=V\left(1+\frac{A^{2}}{\bar{A}^{2}}(\langle U\rangle-1)\right) \simeq\left(1-h \frac{A^{2}}{\bar{A}^{2}}\left(1-\exp \left(-\frac{1}{d}\right)\right)\right) V
$$

Similarly, in (73) we can replace $A P K^{\alpha} U-A U V$ by its lowest order approximation, that is

$$
A P K^{\alpha} U-A U V=A U\left(P K^{\alpha}-P^{1+\gamma} K^{\alpha}\right)
$$

The correction associated to this term is of order $\gamma \frac{A^{2}}{A^{2}}$ and can be neglected. Moreover, by the same argument, up to corrections of order $\gamma \frac{A^{2}}{A^{2}}$, one has :

$$
\frac{A P K^{\alpha} U+\frac{\bar{A}^{2} A^{2}}{\left(A^{2} U^{2}+\bar{A}^{2}\right)^{2}} A U V}{\delta\left(1+\frac{\bar{A}^{2} A^{2}}{\left(A^{2} U^{2}+\bar{A}^{2}\right)^{2}}\right)} \simeq A U \frac{P K^{\alpha}+\frac{\bar{A}^{2} A^{2}}{\left(A^{2} U^{2}+\bar{A}^{2}\right)^{2}} P K^{\alpha}}{\delta\left(1+\frac{\bar{A}^{2} A^{2}}{\left(A^{2} U^{2}+\bar{A}^{2}\right)^{2}}\right)}=A U P K^{\alpha}
$$

and the potential is:

$$
\delta^{2}\left(1+\frac{\bar{A}^{2} A^{2}}{\left(A^{2} U^{2}+\bar{A}^{2}\right)^{2}}\right)\left(K-\frac{A U P K^{\alpha}}{\delta}\right)^{2}
$$

In these formula, $U$ can be estimated by $1-h \exp \left(-\frac{1}{2 d}\right)\left(1-\frac{\exp \left(-\frac{1}{2 d}\right)}{2}\right)$. As a consequence, the equation for $P$ is the same as before with an additional factor $U$, and the equation for $\langle K\rangle_{X}$ is unchanged. We can then use a similar trial function:

$$
P \simeq(K)^{-\frac{\alpha}{1+\gamma}} f(X)
$$

and these equations become:

$$
(f(X))^{1+\gamma}=\bar{\kappa} \int P_{2}\left(K_{2}\right)^{\alpha} P_{3} \exp \left(-\left(\left|X-X_{2}\right|+\left|X_{2}-X_{3}\right|\right)\right)\left|\Psi\left(K_{3}, P_{3}, X_{3}, \theta\right)\right|^{2}\left|\Psi\left(K_{2}, P_{2}, X_{2}, \theta\right)\right|^{2}
$$

with:

$$
\begin{gathered}
\bar{\kappa}=\kappa\left(1-h \frac{A^{2}}{\bar{A}^{2}}\left(1-\exp \left(-\frac{1}{d}\right)\right)\right) \\
\langle K\rangle_{X} \simeq\left(\frac{A}{\delta} f(X)\right)^{\frac{1+\gamma}{1+\gamma(1-\alpha)}}\left(1-\frac{\kappa}{4 d^{2}} \int\langle K\rangle_{X_{2}}^{\frac{\alpha \gamma}{1+\gamma}}\langle K\rangle_{X_{3}}^{-\frac{\alpha}{1+\gamma}} f\left(X_{3}\right)\left(f\left(X_{2}\right)\right)^{-\gamma} \exp \left(-\left(\frac{\left|X-X_{2}\right|}{d}+\frac{\left|X-X_{3}\right|}{d}\right)\right)\right)
\end{gathered}
$$

And the resolution is identical to the case $A \rightarrow 0$. We thus find:

$$
f(X)=D \exp \left(-\frac{|X|}{(1+\gamma) d}\right)
$$

with:

$D=\left(\frac{1+\gamma(1-\alpha)}{(2-\alpha) \bar{\kappa}}\left(\left(\frac{2-\alpha}{1+\gamma(1-\alpha)}\right)^{2}-1\right)\left(\frac{A}{\delta}\left(1-h \exp \left(-\frac{1}{2 d}\right)\left(1-\frac{\exp \left(-\frac{1}{2 d}\right)}{2}\right)\right)\right)^{\frac{\alpha(1-\gamma)}{1+\gamma(1-\alpha)}}\right)^{\frac{1+\gamma(1-\alpha)}{\left(1-\gamma^{2}\right)(1-\alpha)}}$

and for $\langle K\rangle_{X}$ :

$$
\langle K\rangle_{X}=\left(\frac{A}{\delta} f(X)\left(1-h \exp \left(-\frac{|X|}{d}\right)\left(1-\frac{\cosh \frac{X}{d}}{\exp \left(\frac{1}{d}\right)}\right)\right)\right)^{\frac{1+\gamma}{1+\gamma(1-\alpha)}}
$$

with:

$$
\begin{aligned}
h & =\frac{\bar{h}}{\left(1-\frac{A^{2}}{A^{2}} \bar{h}\left(1-\exp \left(-\frac{1}{d}\right)\right)\right)} \\
\bar{h} & =(1+\gamma(1-\alpha))\left(\frac{1+\gamma(1-\alpha)}{2(2-\alpha)}\left(\left(\frac{2-\alpha}{1+\gamma(1-\alpha)}\right)^{2}-1\right)\right)\left(\frac{3}{2}-\frac{e^{-\frac{1}{d}}}{2}\right)
\end{aligned}
$$


to the first order in $\frac{A^{2}}{A^{2}}$. Formula (69) for the price becomes:

$$
P=\frac{\left(\frac{1+\gamma(1-\alpha)}{(2-\alpha) \kappa}\left(\left(\frac{2-\alpha}{1+\gamma(1-\alpha)}\right)^{2}-1\right)\right)^{\frac{1}{1-\gamma}} \exp \left(-\frac{(1-\alpha)|X|}{d(1+\gamma(1-\alpha))}\right)}{\left(\frac{K}{\langle K\rangle_{X}}\right)^{\frac{\alpha}{1+\gamma}}\left(\left(\frac{1-h \exp \left(-\frac{|X|}{d}\right)\left(1-\frac{\cosh \frac{X}{d}}{\exp \left(\frac{1}{d}\right)}\right)}{1-h \exp \left(-\frac{1}{2 d}\right)\left(1-\frac{\exp \left(-\frac{1}{2 d}\right)}{2}\right)}\right)\right)^{\frac{\alpha}{1+\gamma(1-\alpha)}}}
$$

\subsection{Appendix 3}

For $\rho>0$, the contribution of the mean cnfgrtn added to the fluctuations. Previous computations are valid, but average values have to be computed in states including the fundamental's contribution in the state $\Psi_{0}+\Psi$. We first consider the state $\Psi_{0}(K, P, X, \theta)$ only, and in this state, we replace $P$, find the equations for $f(X)$ and $\langle K\rangle_{X}$. Then, we compute the form of fundamental $\Psi_{0}$ and the value of $\rho$. We then compute the correction due to $\Psi(K, P, X, \theta)$ and find the effective action.

\subsubsection{Equations for $P, f(X)$ and $\langle K\rangle_{X}$ in the state $\Psi_{0}$}

In the state $\Psi_{0}(K, P, X, \theta)$, the previous equations defining the potential, $P$ and $\langle K\rangle_{X}$ are the same as before. We will include directly the first order corrections in $\frac{A^{2}}{A^{2}}$ since it amounts, as in Appendix 2, to replace $\kappa$ by $\bar{\kappa}$ (see 75) in the equation for $f(X)$.

Now, to compute $f(X)$ we first find the average of $K$, denoted $\langle K\rangle_{X}$ computed in a state $\rho \Psi_{0}(K, X)$, which amounts to replace now, given our order of approximations, for $\Psi_{0}(K, X)$

$$
\kappa \rightarrow \kappa \rho^{4}
$$

One sets again:

$$
P=(K)^{-\frac{\alpha}{1+\gamma}} f(X)
$$

The equation for $f(X)$ is the same as before

$$
f^{1+\gamma}(X)=\bar{\kappa} \rho^{4} \int P_{2}\left(K_{2}\right)^{\alpha} P_{3} \exp \left(-\left(\left|X-X_{2}\right|+\left|X_{2}-X_{3}\right|\right)\right)\left|\Psi_{0}\left(K_{2}, P_{2}, X_{2}, \theta\right)\right|^{2}\left|\Psi_{0}\left(K_{3}, P_{3}, X_{3}, \theta\right)\right|^{2}
$$

where:

$$
\bar{\kappa}=\kappa\left(1-h \frac{A^{2}}{\bar{A}^{2}}\left(1-\exp \left(-\frac{1}{d}\right)\right)\right)
$$

includes the first order corrections in $\frac{A^{2}}{A^{2}}$. The equation for $\langle K\rangle_{X}$ is still valid in first approximation:

$$
\langle K\rangle_{X}=\left(\frac{A}{\delta} f(X)\right)^{\frac{1+\gamma}{1+\gamma(1-\alpha)}}\left(\begin{array}{c}
1-\kappa \rho^{4} \int\left(K_{2}\right)^{\frac{\alpha \gamma}{1+\gamma}}\left(K_{3}\right)^{-\frac{\alpha}{1+\gamma}} f\left(X_{3}\right)\left(f\left(X_{2}\right)\right)^{-\gamma} \times \\
\times \exp \left(-\left(\frac{\left|X-X_{2}\right|}{d}+\frac{\left|X-X_{3}\right|}{d}\right)\right)\left|\Psi_{0}\left(K_{2}, P_{2}, X_{2}, \theta\right)\right|^{2}\left|\Psi_{0}\left(K_{3}, P_{3}, X_{3}, \theta\right)\right|^{2}
\end{array}\right)^{\frac{1+\gamma}{1+\gamma(1-\alpha)}}
$$

For the same reasons as in Appendix 2, the state $\Psi_{0}^{\dagger}(K, X)$ is centered around $\langle K\rangle_{X}$. Thus, the equation for $f(X)$ is thus:

$$
(f(X))^{1+\gamma} \simeq \bar{\kappa} \rho^{4} \int\langle K\rangle_{X_{2}}^{\frac{\alpha(\gamma-1)}{1+\gamma}}\left(f\left(X_{2}\right)\right)^{2} G\left(X_{2}, X_{2}\right) \exp \left(-\left(\frac{\left|X-X_{2}\right|}{d}\right)\right)
$$

Moreover, in first approximation, the defining equation for the $X$ part of $\Psi_{0}(K, X)$ is an oscillator with $\kappa_{0}<<1$. The distribution for $X$ can thus be considered as uniform. The equations for $f(X)$ and for $K$ are thus similar to that obtained in Appendix 2, apart from a $\rho^{4}$ factor and write:

$$
(f(X))^{1+\gamma} \simeq \frac{\bar{\kappa} \rho^{4}}{2} \int\langle K\rangle_{X_{2}}^{\frac{\alpha(\gamma-1)}{1+\gamma}}\left(f\left(X_{2}\right)\right)^{2} \exp \left(-\frac{\left|X-X_{2}\right|}{d}\right)
$$


$\langle K\rangle_{X} \simeq\left(\frac{A}{\delta} f(X)\right)^{\frac{1+\gamma}{1+\gamma(1-\alpha)}}\left(1-\frac{\kappa \rho^{4}}{4} \int\langle K\rangle_{X_{2}}^{\frac{\alpha \gamma}{1+\gamma}}\langle K\rangle_{X_{3}}^{-\frac{\alpha}{1+\gamma}} f\left(X_{3}\right)\left(f\left(X_{2}\right)\right)^{-\gamma} \exp \left(-\left(\frac{\left|X-X_{2}\right|}{d}+\frac{\left|X-X_{3}\right|}{d}\right)\right)\right)$

Again, the form for $\langle K\rangle_{X}$ is postulated:

$$
\langle K\rangle_{X}=\left(\frac{A}{\delta} f(X)\left(1-h \exp \left(-g \frac{|X|}{d}\right)\left(1-\frac{\cosh \frac{X}{d}}{\exp \left(\frac{1}{d}\right)}\right)\right)\right)^{\frac{1+\gamma}{1+\gamma(1-\alpha)}}
$$

the resolution is thus similar to Appendix 2 and one finds:

$$
\begin{aligned}
f(X)= & D \exp \left(-\frac{|X|}{1+\gamma}\right) \\
= & \left(\frac{A}{\delta}\left(1-h \exp \left(-\frac{1}{2 d}\right)\left(1-\frac{\exp \left(-\frac{1}{2 d}\right)}{2}\right)\right)\right)^{\frac{\alpha}{(1-\alpha)(\gamma+1)}} \\
& \times\left(\frac{1+\gamma(1-\alpha)}{(2-\alpha) \bar{\kappa} \rho^{4}}\left(\left(\frac{2-\alpha}{1+\gamma(1-\alpha)}\right)^{2}-1\right)\right)^{\frac{1+\gamma(1-\alpha)}{\left(1-\gamma^{2}\right)(1-\alpha)}} \exp \left(-\frac{|X|}{d(1+\gamma)}\right)
\end{aligned}
$$

where:

$$
\begin{aligned}
h & =\frac{\bar{h}}{\left(1-\frac{A^{2}}{A^{2}} \bar{h}\left(1-\exp \left(-\frac{1}{d}\right)\right)\right)} \\
\bar{h} & =(1+\gamma(1-\alpha))\left(\frac{1+\gamma(1-\alpha)}{2(2-\alpha)}\left(\left(\frac{2-\alpha}{1+\gamma(1-\alpha)}\right)^{2}-1\right)\right)\left(\frac{3}{2}-\frac{e^{-\frac{1}{d}}}{2}\right)
\end{aligned}
$$

And for the price:

$$
P=\frac{\left(\frac{1+\gamma(1-\alpha)}{(2-\alpha) \bar{\kappa} \rho^{4}}\left(\left(\frac{2-\alpha}{1+\gamma(1-\alpha)}\right)^{2}-1\right)\right)^{\frac{1}{1-\gamma}} \exp \left(-\frac{(1-\alpha)|X|}{d(1+\gamma(1-\alpha))}\right)}{\left(\frac{K}{\langle K\rangle_{X}}\right)^{\frac{\alpha}{1+\gamma}}\left(\left(\frac{1-h \exp \left(-\frac{|X|}{d}\right)\left(1-\frac{\cosh \frac{X}{d}}{\exp \left(\frac{1}{d}\right)}\right)}{1-h \exp \left(-\frac{1}{2 d}\right)\left(1-\frac{\exp \left(-\frac{1}{2 d}\right)}{2}\right)}\right)\right)^{\frac{\alpha}{1+\gamma(1-\alpha)}}}
$$

\subsubsection{Computation of $\rho$ and $\Psi_{0}$}

The value of $\rho$ can now be computed. As said before, this phase is possible approximatively for:

$$
\kappa_{1}^{2}-2 \kappa_{0}^{\frac{1}{2}} \kappa_{2}>0
$$

In this phase, taking into account only the contribution in $X$, then $\rho$ can be found approximatively by:

$$
\rho \simeq \frac{\kappa_{1}+\sqrt{\kappa_{1}^{2}-2 \kappa_{0}^{\frac{1}{2}} \kappa_{2}}}{2 \kappa_{2}}
$$

with $\frac{\partial^{2} S}{\partial \rho^{2}}>0$. The value of $\rho$ is found more precisely by writing the equation for the state $\Psi_{0}(K, X)$. For the part in $X$ :

$$
\begin{aligned}
& \sigma_{X}^{2}(\nabla \Psi(x))^{2}+\frac{\kappa_{0}}{\sigma_{X}^{2}} \Psi^{\dagger}(x)(x-\langle x\rangle)^{2} \Psi(x)+\left(\Psi^{\dagger}(x) \Psi(x)\right) V_{1}(|x-y|)\left(\Psi^{\dagger}(y) \Psi(y)\right) \\
& +\left(\Psi^{\dagger}(x) \Psi(x)\right) V_{2}(|x-y|,|x-z|,|y-z|)\left(\Psi^{\dagger}(y) \Psi(y)\right)\left(\Psi^{\dagger}(z) \Psi(z)\right)
\end{aligned}
$$


where:

$$
\begin{aligned}
V_{1}(|x-y|) & =-\kappa_{1} \frac{K K^{\prime} \exp \left(-\chi_{1}|x-y|\right)}{\langle K\rangle_{x}\langle K\rangle_{y}} \\
V_{2}(|x-y|,|x-z|,|y-z|) & =\kappa_{2} \exp \left(-\chi_{2}|x-y|-\chi_{2}|x-z|-\chi_{2}|y-z|\right)
\end{aligned}
$$

Considering the $X$ part of $\frac{\delta}{\delta \Psi(x)}$ :

$-\sigma_{X}^{2} \nabla^{2} \Psi(x)+\frac{\kappa_{0}}{\sigma_{X}^{2}}(x-\langle x\rangle)^{2} \Psi(x)+V_{1}(|x-y|)|\Psi(y)|^{2} \Psi(x)+V_{2}(|x-y|,|x-z|,|y-z|)|\Psi(y)|^{2}|\Psi(z)|^{2} \Psi(x)$

It can be approximated by computing the potentials $V_{1}(|x-y|)$ and $V_{2}(|x-y|,|x-z|,|y-z|)$ in the fundamental:

$$
\begin{aligned}
& \int \kappa_{1} \frac{K K^{\prime} \exp \left(-\chi_{1}|x-y|\right)}{\langle K\rangle_{x}\langle K\rangle_{y}}\left|\Psi\left(K^{\prime}, y\right)\right|^{2} d y \\
\rightarrow & \int \kappa_{1} \frac{K K^{\prime} \exp \left(-\chi_{1}|x-y|\right)}{\langle K\rangle_{x}\langle K\rangle_{y}}\left|\Psi_{0}\left(K^{\prime}, y\right)\right|^{2} d y \\
\simeq & \kappa_{1} \exp \left(-\chi_{1}|x|\right) \frac{K}{\langle K\rangle_{x}} \rho^{2} \\
\simeq & \kappa_{1} \exp \left(-\chi_{1}|x|\right) \rho^{2}
\end{aligned}
$$

Similarly, the second part of the potential is replaced by:

$$
\begin{aligned}
& \kappa_{2} \exp \left(-\chi_{2}|x-y|-\chi_{2}|x-z|-\chi_{2}|y-z|\right)\left|\Psi\left(K^{\prime}, y\right)\right|^{2}\left|\Psi\left(K^{\prime \prime}, z\right)\right|^{2} \\
\rightarrow & \int \kappa_{2} \exp \left(-\chi_{2}|x-y|-\chi_{2}|x-z|-\chi_{2}|y-z|\right)\left|\Psi_{0}(K, y)\right|^{2}\left|\Psi_{0}(K, z)\right|^{2} d y d z \\
\simeq & \kappa_{2} \exp \left(-2 \chi_{2}|x|\right) \rho^{4}
\end{aligned}
$$

Moreover in first approximation, we can find the fundamental by taking the expectation values of the variable $x$ :

$$
\langle x\rangle=0
$$

for an overall expression:

$$
-\sigma_{X}^{2} \nabla^{2} \Psi(x)+\kappa_{0} x^{2} \Psi(x)-\kappa_{1} \frac{K}{\langle K\rangle_{x}} \exp \left(-\chi_{1}|x|\right) \Psi(K, x) \rho^{2}+\kappa_{2} \exp \left(-2 \chi_{2}|x|\right) \Psi(K, x) \rho^{4}+\alpha \Psi(x)
$$

Adding the $K$ a part of the action, and considering $\chi_{1}<<1, \chi_{2}<<1$, at the lowest order in $\chi_{1}$ and $\chi_{2}$, the fundamental equation is:

$$
\begin{aligned}
& 0=\left(-\sigma_{X}^{2} \nabla_{X}^{2}-\sigma^{2} \nabla_{K}^{2}-\vartheta^{2} \nabla_{\theta}^{2}\right) \Psi_{0}(K, X, \theta) \\
& +\left(\left(\delta^{2}+\frac{\bar{A}^{2} A^{2}}{\left(A^{2} U^{2}+\bar{A}^{2}\right)^{2}}\right)\left(K-\langle K\rangle_{X}\right)^{2}+\kappa_{0} x^{2}+\left(\rho^{4} \kappa_{2}-\rho^{2} \kappa_{1}+\frac{1}{\vartheta^{2}}+\alpha\right)\right) \Psi_{0}(K, X, \theta)
\end{aligned}
$$

The fourier transform in $\theta$ shows that the fundamental does not depend on $\theta$. Eigenstates of the operator leads to look for a fundamental of the form $N \Psi_{0}(X) \Psi_{0}(K)$. We assume $\theta<\Theta$ with $\Theta>>1$, so that the integral over $\theta$ exists.

The normalization factor $N$ ensures that $N \Psi_{0}(X) \Psi_{0}(K)$ has norm $\rho^{2}$. Then $\Psi_{0}(X)$ and $\Psi_{0}(K)$ can be searched as fundamental states of oscillators with eigenstates

$$
\kappa_{0}^{\frac{1}{2}} \text { and } \sigma \sqrt{\delta^{2}+\frac{\bar{A}^{2} A^{2}}{\left(A^{2} U^{2}+\bar{A}^{2}\right)^{2}}}
$$


and this leads to the relation:

$$
0=\alpha+\frac{1}{\vartheta^{2}}+\frac{1}{2} \kappa_{0}^{\frac{1}{2}}-\kappa_{1} \rho^{2}+\kappa_{2} \rho^{4}+\frac{\sigma \sqrt{\delta^{2}+\frac{\bar{A}^{2} A^{2}}{\left(A^{2} U^{2}+\bar{A}^{2}\right)^{2}}}}{2}
$$

with:

$$
\begin{aligned}
U & =\left(1-D^{1+\gamma} \int \exp \left(-\frac{\left|X_{3}\right|}{(1+\gamma)}\right) \exp \left(\frac{\gamma\left|X_{2}\right|}{(1+\gamma)}\right) \exp \left(-\left(\left|X-X_{2}\right|+\left|X-X_{3}\right|\right)\right)\right) \\
& \simeq 1-h\left(1-\exp \left(-\frac{1}{d}\right)\right)
\end{aligned}
$$

whose solution, with with $\frac{\partial^{2}}{\partial \rho^{2}}>0$, is:

$$
\rho^{2}=\frac{\kappa_{1}+\sqrt{\kappa_{1}^{2}-2 \kappa_{2}\left(2 \alpha+\frac{2}{\vartheta^{2}}+\sqrt{\kappa_{0}}+\sigma \sqrt{\delta^{2}+\frac{\bar{A}^{2} A^{2}}{\left(A^{2} U^{2}+\bar{A}^{2}\right)^{2}}}\right)}}{2 \kappa_{2}}
$$

and the eigenstate:

$$
\Psi_{0}(K, X)=N \exp \left(-\frac{\kappa_{0}^{-\frac{1}{2}} X^{2}}{2}\right) \exp \left(-\frac{\sqrt{\delta^{2}+\frac{\bar{A}^{2} A^{2}}{\left(A^{2} U^{2}+\bar{A}^{2}\right)^{2}}}\left(K-\langle K\rangle_{X}\right)^{2}}{2 \sigma}\right)
$$

where $N$ is the normalisation factor:

$$
N=\frac{\rho}{2 \pi \sqrt{\Theta \kappa_{0}^{-\frac{1}{2}} \sigma \sqrt{\delta^{2}+\frac{\bar{A}^{2} A^{2}}{\left(A^{2} U^{2}+\bar{A}^{2}\right)^{2}}}}}
$$

which completes the computations for $P,\langle K\rangle$, and the potential.

For later purpose, we also derive the corrections to these results at the second order in $\chi_{1}$ and $\chi_{2}$. At this order, expanding the exponentials in $\chi_{1}$ and $\chi_{2}$ yields the potential of the $X$ part of the action:

$$
\begin{aligned}
& \frac{1}{2} \kappa_{0} x^{2}+\kappa_{1} \chi_{1}|x| \frac{K}{\langle K\rangle_{x}} \rho^{2}-2 \kappa_{2} \chi_{2}|x| \rho^{4}-\frac{\kappa_{1}}{2} \chi_{1}^{2} x^{2}\left(\frac{K}{\langle K\rangle_{x}} \rho^{2}\right)^{2}+2 \kappa_{2} \chi_{2}^{2} x^{2} \rho^{8} \\
\rightarrow & \frac{1}{2} \kappa_{0}\left(x+\frac{\operatorname{sgn}(x)}{\kappa_{0}}\left(\chi_{1} \kappa_{1} \frac{K}{2\langle K\rangle_{x}} \rho^{2}-2 \chi_{2} \kappa_{2} \rho^{4}\right)\right)^{2}-\frac{1}{2 \kappa_{0}}\left(\chi_{1} \kappa_{1} \frac{K}{2\langle K\rangle_{x}} \rho^{2}-2 \chi_{2} \kappa_{2} \rho^{4}\right)^{2} \\
& +\left(-\frac{\kappa_{1} \chi_{1}^{2} \rho^{2}}{2}+2 \chi_{2}^{2} \kappa_{2} \rho^{4}\right) x^{2} \\
\simeq & \frac{1}{2}\left(\kappa_{0}+\left(-\frac{\kappa_{1} \chi_{1}^{2} \rho^{2}}{2}+2 \chi_{2}^{2} \kappa_{2} \rho^{4}\right)\right)\left(x+\frac{\operatorname{sgn}(x)}{\kappa_{0}}\left(\chi_{1} \kappa_{1} \frac{K}{2\langle K\rangle_{x}} \rho^{2}-2 \chi_{2} \kappa_{2} \rho^{4}\right)\right)^{2} \\
& -\frac{1}{2 \kappa_{0}}\left(\chi_{1} \kappa_{1} \frac{K}{2\langle K\rangle_{x}} \rho^{2}-2 \chi_{2} \kappa_{2} \rho^{4}\right)^{2}
\end{aligned}
$$


so that, the fundamental equation becomes:

$$
\begin{aligned}
& 0=\left(-\sigma_{X}^{2} \nabla_{X}^{2}-\sigma^{2} \nabla_{K}^{2}-\vartheta^{2} \nabla_{\theta}^{2}\right) \Psi_{0}(K, X, \theta) \\
& +\kappa_{0}\left(1-\frac{\chi_{1}^{2} \kappa_{1} \rho^{2}}{2 \kappa_{0}}+2 \chi_{2} \frac{\kappa_{2}}{\kappa_{0}} \rho^{4}\right)\left(x+\operatorname{sgn}(x)\left(\chi_{1} \kappa_{1} \frac{K}{2\langle K\rangle_{x}} \rho^{2}-\chi_{2}^{2} \kappa_{2} \rho^{4}\right)\right)^{2} \Psi_{0}(K, X, \theta) \\
& +\left(\left(\delta^{2}+\frac{\bar{A}^{2} A^{2}}{\left(A^{2} U^{2}+\bar{A}^{2}\right)^{2}}\right)\left(K-\langle K\rangle_{X}\right)^{2}\right) \Psi_{0}(K, X, \theta) \\
& +\left(\rho^{4} \kappa_{2}-\rho^{2} \kappa_{1}-\left(\chi_{1} \kappa_{1} \frac{K}{2\langle K\rangle_{x}} \rho^{2}-\chi_{2} \kappa_{2} \rho^{4}\right)^{2}+\frac{1}{\vartheta^{2}}+\alpha\right) \Psi_{0}(K, X, \theta)
\end{aligned}
$$

Again, we look for a fundamental of the form $N \Psi_{0}(X) \Psi_{0}(K)$. The operators in $X$ and $K$ are harmonic oscillators with frequencies:

$$
\begin{aligned}
\kappa_{0}^{\frac{1}{2}} \sqrt{1-\frac{\chi_{1}^{2} \kappa_{1} \rho^{2}}{2 \kappa_{0}}+2 \chi_{2}^{2} \frac{\kappa_{2}}{\kappa_{0}} \rho^{4}} \text { and } \sigma \sqrt{\delta^{2}+\frac{\bar{A}^{2} A^{2}}{\left(A^{2} U^{2}+\bar{A}^{2}\right)^{2}}} \\
\Psi_{0}(X)=\exp \left(-\frac{\kappa_{0}^{-\frac{1}{2}}\left(X+\left(\chi_{1} \kappa_{1} \frac{K}{2\langle K\rangle_{X}} \rho^{2}-\chi_{2} \kappa_{2} \rho^{4}\right)\right)^{2}}{2}\right) H(x) \\
+\exp \left(-\frac{\kappa_{0}^{-\frac{1}{2}}\left(X-\left(\chi_{1} \kappa_{1} \frac{K}{2\langle K\rangle_{X}} \rho^{2}-\chi_{2} \kappa_{2} \rho^{4}\right)\right)^{2}}{2}\right) H(-x) \\
\equiv \quad \bar{\Psi}_{0}\left(X+\left(\chi_{1} \kappa_{1} \frac{K}{2\langle K\rangle_{X}} \rho^{2}-\chi_{2} \kappa_{2} \rho^{4}\right)\right) H(X)+\bar{\Psi}_{0}\left(X-\left(\chi_{1} \kappa_{1} \frac{K}{2\langle K\rangle_{X}} \rho^{2}-\chi_{2} \kappa_{2} \rho^{4}\right)\right) H(-X) \\
\Psi_{0}(K)=\exp \left(-\frac{\sqrt{\delta^{2}+\frac{\bar{A}^{2} A^{2}}{\left(A^{2} U^{2}+\bar{A}^{2}\right)^{2}}}\left(K-\langle K\rangle_{X}\right)^{2}}{2 \sigma}\right)
\end{aligned}
$$

and the fundamental state writes:

$$
\begin{aligned}
\Psi_{0}(K, X)= & \rho N\left[\bar{\Psi}_{0}(X+\delta X) H(X)+\bar{\Psi}_{0}(X-\delta X) H(-X)\right] \\
& \times \exp \left(-\frac{\sqrt{\delta^{2}+\frac{\bar{A}^{2} A^{2}}{\left(A^{2} U^{2}+\bar{A}^{2}\right)^{2}}}\left(K-\langle K\rangle_{X}\right)^{2}}{2 \sigma}\right)
\end{aligned}
$$

where $N$ is a normalisation factor:

$$
N=\frac{1}{2 \pi \sqrt{\kappa_{0}^{-\frac{1}{2}} \sigma \sqrt{\delta^{2}+\frac{\bar{A}^{2} A^{2}}{\left(A^{2} U^{2}+\bar{A}^{2}\right)^{2}}}}}
$$

and:

$$
\delta X=\left(\chi_{1} \kappa_{1} \frac{K}{2\langle K\rangle} \rho^{2}-\chi_{2} \kappa_{2} \rho^{4}\right)
$$


with $\rho$ satisfying the condition:

$$
\begin{aligned}
0= & \alpha+\frac{1}{\vartheta^{2}}+\frac{1}{2} \kappa_{0}^{\frac{1}{2}} \sqrt{1-\frac{\chi_{1}^{2} \kappa_{1} \rho^{2}}{2}+2 \chi_{2}^{2} \kappa_{2} \rho^{4}} \\
& -\kappa_{1} \rho^{2}+\kappa_{2} \rho^{4}-\left(\chi_{1} \kappa_{1} \frac{K}{2\langle K\rangle_{X}} \rho^{2}-\chi_{2} \kappa_{2} \rho^{4}\right)^{2}+\frac{\sigma \sqrt{\delta^{2}+\frac{\bar{A}^{2} A^{2}}{\left(A^{2} U^{2}+\bar{A}^{2}\right)^{2}}}}{2}
\end{aligned}
$$

\subsubsection{Contribution of $\Psi$}

Now, the replacement $\Psi\left(K_{3}, P_{3}, X_{3}, \theta\right) \rightarrow \Psi_{0}\left(K_{3}, P_{3}, X_{3}, \theta\right)+\delta \Psi\left(K_{3}, P_{3}, X_{3}, \theta\right)$ for $V$ can be performed in the following way. For any quantity, $(K)^{-\frac{\alpha}{1+\gamma}}, f(X)$ the expectations:

$$
\left(\Psi_{0}^{\dagger}\left(K_{3}, P_{3}, X_{3}, \theta\right)+\delta \Psi^{\dagger}\left(K_{3}, P_{3}, X_{3}, \theta\right)\right)\left(\begin{array}{c}
(K)^{-\frac{\alpha}{1+\gamma}} \\
f(X)
\end{array}\right)\left(\Psi_{0}\left(K_{3}, P_{3}, X_{3}, \theta\right)+\delta \Psi\left(K_{3}, P_{3}, X_{3}, \theta\right)\right)
$$

are approximatively:

$$
\Psi_{0}^{\dagger}\left(K_{3}, P_{3}, X_{3}, \theta\right)\left(\begin{array}{c}
(K)^{-\frac{\alpha}{1+\gamma}} \\
f(X)
\end{array}\right) \Psi_{0}\left(K_{3}, P_{3}, X_{3}, \theta\right)+\delta \Psi^{\dagger}\left(K_{3}, P_{3}, X_{3}, \theta\right)\left(\begin{array}{c}
(K)^{-\frac{\alpha}{1+\gamma}} \\
f(X)
\end{array}\right) \delta \Psi\left(K_{3}, P_{3}, X_{3}, \theta\right)
$$

since, given their form, $(K)^{-\frac{\alpha}{1+\gamma}}$ and $f(X)$ can be considered as close to their average $\langle K\rangle^{-\frac{\alpha}{1+\gamma}}$ and $f(\langle X\rangle)$ and thus

$$
\left\langle\Psi_{0}^{\dagger}\left(K_{3}, P_{3}, X_{3}, \theta\right)\left(\begin{array}{c}
(K)^{-\frac{\alpha}{1+\gamma}} \\
f(X)
\end{array}\right), \delta \Psi\left(K_{3}, P_{3}, X_{3}, \theta\right)\right\rangle \simeq 0
$$

for a perturbation $\delta \Psi(K, X)$ orthogonal to $\Psi_{0}(K, X)$. As a consequence the second order development of the potential terms are:

$$
\begin{aligned}
& \Psi_{0}^{\dagger}(K, X)\left(f^{1+\gamma}(X)-\kappa \int P_{2}\left(K_{2}\right)^{\alpha} P_{3} \exp \left(-\frac{\left|X-X_{2}\right|+\left|X_{2}-X_{3}\right|}{d}\right)\left|\Psi_{0}\left(K_{2}, X_{2}\right)\right|^{2}\left|\Psi_{0}\left(K_{3}, X_{3}\right)\right|^{2}\right)^{2} \\
& +\delta \Psi^{\dagger}(K, X)\left(f^{1+\gamma}(X)-\kappa \int P_{2}\left(K_{2}\right)^{\alpha} P_{3} \exp \left(-\frac{\left|X-X_{2}\right|+\left|X_{2}-X_{3}\right|}{d}\right)\left|\Psi_{0}\left(K_{2}, X_{2}\right)\right|^{2}\left|\Psi_{0}\left(K_{3}, X_{3}\right)\right|^{2}\right) \delta \Psi(K, X) \\
& -2 \kappa \Psi_{0}^{\dagger}(K, X)\left(\int \Psi_{0}^{\dagger}\left(K_{3}, X_{3}\right) \delta \Psi^{\dagger}\left(K_{2}, X_{2}\right) P_{2}\left(K_{2}\right)^{\alpha} P_{3} \exp \left(-\frac{\left|X-X_{2}\right|+\left|X_{2}-X_{3}\right|}{d}\right) \delta \Psi\left(K_{2}, X_{2}\right) \Psi_{0}\left(K_{3}, X_{3}\right)\right. \\
& \left.+\int \Psi_{0}^{\dagger}\left(K_{2}, X_{2}\right) \delta \Psi^{\dagger}\left(K_{3}, X_{3}\right) P_{2}\left(K_{2}\right)^{\alpha} P_{3} \exp \left(-\frac{\left|X-X_{2}\right|+\left|X_{2}-X_{3}\right|}{d}\right) \delta \Psi\left(K_{3}, X_{3}\right) \Psi_{0}\left(K_{2}, X_{2}\right)\right) \\
& \times\left(f^{1+\gamma}(X)-\kappa \int P_{2}\left(K_{2}\right)^{\alpha} P_{3} \exp \left(-\frac{\left|X-X_{2}\right|+\left|X_{2}-X_{3}\right|}{d}\right)\left|\Psi_{0}\left(K_{2}, X_{2}\right)\right|^{2}\left|\Psi_{0}\left(K_{3}, X_{3}\right)\right|^{2}\right) \Psi_{0}(K, X)
\end{aligned}
$$

where, as before, we define $f(X)=P K^{-\frac{\alpha}{1+\gamma}}$. The first term is the action part for $\Psi_{0}(K, X)$, it can be discarded (and in fact is equal to zero given the constraint).

Given that both for $\Psi_{0}$ and $\delta \Psi, K$ is centered around $\langle K\rangle_{X, 0}$ and $\langle K\rangle_{X}$, and that for both state we may consider the $X$ ditribution as uniform and, as a first approximation, we set:

$$
\begin{aligned}
G_{0}\left(X_{i}, X_{i}\right) & =\rho^{2} \Psi_{0}^{\dagger}\left(\langle K\rangle_{X_{i}, 0}, X_{i}\right) \Psi_{0}\left(\langle K\rangle_{X_{i}, 0}, X_{i}\right) \\
G\left(X_{i}, X_{i}\right) & =G\left(\left(\langle K\rangle_{X_{i}}, X_{i}\right),\left(\langle K\rangle_{X_{j}}, X_{j}\right)\right)
\end{aligned}
$$

and:

$$
\begin{aligned}
f_{0}(X) & =\int P K^{-\frac{\alpha}{1+\gamma}}\left|\Psi_{0}(K, X)\right|^{2} d K \simeq P K^{-\frac{\alpha}{1+\gamma}}\left|\Psi_{0}\left(\langle K\rangle_{X, 0}, X\right)\right|^{2} \\
f_{0}^{1+\gamma}(X) & =\int P K^{-\frac{\alpha}{1+\gamma}}\left|\Psi_{0}(K, X)\right|^{2} d K \simeq P^{1+\gamma} K^{-\alpha}\left|\Psi_{0}\left(\langle K\rangle_{X, 0}, X\right)\right|^{2}
\end{aligned}
$$


so that the last term rewrites:

$$
\begin{aligned}
& -\kappa \int G_{0}\left(X_{2}, X_{2}\right) G\left(X_{3}, X_{3}\right)\langle K\rangle_{X_{2}, 0}^{\frac{\alpha \gamma}{1+\gamma}}\langle K\rangle_{X_{3}}^{-\frac{\alpha}{1+\gamma}} f\left(X_{3}\right) f_{0}\left(X_{2}\right) \exp \left(-\frac{\left|X-X_{2}\right|+\left|X_{2}-X_{3}\right|}{d}\right) \\
& \times\left(\left(f_{0}(X)\right)^{1+\gamma}-\kappa \int G_{0}\left(X_{2}, X_{2}\right) G_{0}\left(X_{3}, X_{3}\right)\langle K\rangle_{X_{2}, 0}^{\frac{\alpha \gamma}{1+\gamma}}\langle K\rangle_{X_{3}, 0}^{-\frac{\alpha}{1+\gamma}} f_{0}\left(X_{3}\right) f_{0}\left(X_{2}\right) \exp \left(-\frac{\left|X-X_{2}\right|+\left|X_{2}-X_{3}\right|}{d}\right)\right) \\
& \times G_{0}(X, X) \\
& -\kappa \int G\left(X_{2}, X_{2}\right) G_{0}\left(X_{3}, X_{3}\right)\langle K\rangle_{X_{2}}^{\frac{\alpha \gamma}{1+\gamma}}\langle K\rangle_{X_{3}, 0}^{-\frac{\alpha}{1+\gamma}} f_{0}\left(X_{3}\right) f\left(X_{2}\right) \exp \left(-\frac{\left|X-X_{2}\right|+\left|X_{2}-X_{3}\right|}{d}\right) \\
& \times\left(\left(f_{0}(X)\right)^{1+\gamma}-\kappa \int G_{0}\left(X_{2}, X_{2}\right) G_{0}\left(X_{3}, X_{3}\right)\langle K\rangle_{X_{2}, 0}^{\frac{\alpha \gamma}{1+\gamma}}\langle K\rangle_{X_{3}, 0}^{-\frac{\alpha}{1+\gamma}} f_{0}\left(X_{3}\right) f_{0}\left(X_{2}\right) \exp \left(-\frac{\left|X-X_{2}\right|+\left|X_{2}-X_{3}\right|}{d}\right)\right) \\
& \times G_{0}(X, X)
\end{aligned}
$$

As in Appendix 2, we can approximate the integrals by their estimations on the diagonal, and consider in first approximation a uniform distribution for $X$ so that this term rewrites:

$$
\begin{aligned}
& -\kappa \int\left(\langle K\rangle_{X_{2}, 0}^{\frac{\alpha \gamma}{1+\gamma}}\langle K\rangle_{X_{2}}^{-\frac{\alpha}{1+\gamma}}+\langle K\rangle_{X_{2}}^{\frac{\alpha \gamma}{1+\gamma}}\langle K\rangle_{X_{2}, 0}^{-\frac{\alpha}{1+\gamma}}\right) f\left(X_{2}\right) f_{0}\left(X_{2}\right) \\
& \times \int \exp \left(-\left(\frac{\left|X-X_{2}\right|}{d}\right)\right)\left(\left(f_{0}(X)\right)^{1+\gamma}-\kappa \int\langle K\rangle_{X_{2}, 0}^{\frac{\alpha(\gamma-\gamma)}{1+\gamma}}\left(f_{0}\left(X_{2}\right)\right)^{2} \exp \left(-\left(\frac{\left|X-X_{2}\right|}{d}\right)\right)\right)
\end{aligned}
$$

where we used that the $K$ part of $\Psi_{0}$ is peaked around $\langle K\rangle_{X, 0}$, and that the $X$ part is assumed distributed uniformly.

$$
\begin{aligned}
\simeq & -\kappa \int\left(\langle K\rangle_{X, 0}^{\frac{\alpha \gamma}{1+\gamma}}\langle K\rangle_{X}^{-\frac{\alpha}{1+\gamma}}+\langle K\rangle_{X}^{\frac{\alpha \gamma}{1+\gamma}}\langle K\rangle_{X, 0}^{-\frac{\alpha}{1+\gamma}}\right) f(X) f_{0}(X) \\
& \times\left(\left(f_{0}(X)\right)^{1+\gamma}-\kappa \int\langle K\rangle_{X_{2}, 0}^{\frac{\alpha(\gamma-1)}{1+\gamma}}\left(f_{0}\left(X_{2}\right)\right)^{2} \exp \left(-\left(\frac{\left|X-X_{2}\right|}{d}\right)\right)\right) \\
\simeq & 0
\end{aligned}
$$

since the equality

$$
\left(f_{0}(X)\right)^{1+\gamma}-\kappa \int\langle K\rangle_{X_{2}, 0}^{\frac{\alpha(\gamma-1)}{1+\gamma}}\left(f_{0}\left(X_{2}\right)\right)^{2} \exp \left(-\left(\frac{\left|X-X_{2}\right|}{d}\right)\right)=0
$$

holds on $X$. Thus the constraint becomes to the second order:

$\delta \Psi^{\dagger}\left(P^{1+\gamma} K^{\alpha}-\kappa \int G_{0}\left(X_{2}, X_{2}\right) G_{0}\left(X_{3}, X_{3}\right)\langle K\rangle_{X_{2}, 0}^{\frac{\alpha \gamma}{1+\gamma}}\langle K\rangle_{X_{3}, 0}^{-\frac{\alpha}{1+\gamma}} f_{0}\left(X_{3}\right) f_{0}\left(X_{2}\right) \exp \left(-\frac{\left|X-X_{2}\right|+\left|X_{2}-X_{3}\right|}{d}\right)\right)^{2} \delta \Psi$

The identification for $\langle K\rangle_{X}$ :

$$
\Psi^{\dagger}\left(\delta K-A P K^{\alpha}\left(1-\kappa \int \frac{P_{3} \exp \left(-\frac{\left|X_{1}-X_{2}\right|+\left|X_{1}-X_{3}\right|}{d}\right)}{P_{2}^{\gamma}}\left|\Psi_{0}\left(K_{2}, X_{2}\right)\right|^{2}\left|\Psi_{0}\left(K_{3}, X_{3}\right)\right|^{2}\right)\right)^{2} \Psi
$$

becomes at the second order: 


$$
\begin{aligned}
& \Psi_{0}^{\dagger}(K, X)\left(\delta K-A P K^{\alpha}\left(1-\kappa \int \frac{P_{3} \exp \left(-\frac{\left|X-X_{2}\right|+\left|X_{2}-X_{3}\right|}{d}\right)}{P_{2}^{\gamma}}\left|\Psi_{0}\left(K_{2}, X_{2}\right)\right|^{2}\left|\Psi_{0}\left(K_{3}, X_{3}\right)\right|^{2}\right)\right)^{2} \Psi_{0}(K, X) \\
& +\delta \Psi^{\dagger}(K, X)\left(\delta K-A P K^{\alpha}\left(1-\kappa \int \frac{P_{3} \exp \left(-\frac{\left|X-X_{2}\right|+\left|X_{2}-X_{3}\right|}{d}\right)}{P_{2}^{\gamma}}\left|\Psi_{0}\left(K_{2}, X_{2}\right)\right|^{2}\left|\Psi_{0}\left(K_{3}, X_{3}\right)\right|^{2}\right)\right)^{2} \delta \Psi(K, X) \\
& +\Psi_{0}^{\dagger}(K, X)\left(\int \frac{\exp \left(-\frac{\left|X-X_{2}\right|+\left|X_{2}-X_{3}\right|}{d}\right)}{P_{2}^{\gamma}} P_{3}\left|\delta \Psi\left(K_{2}, X_{2}\right)\right|^{2}\left|\Psi_{0}\left(K_{3}, X_{3}\right)\right|^{2}\right. \\
& \left.+\int \frac{\exp \left(-\frac{\left|X-X_{2}\right|+\left|X_{2}-X_{3}\right|}{d}\right)}{P_{2}^{\gamma}} P_{3}\left|\delta \Psi\left(K_{3}, X_{3}\right)\right|^{2}\left|\Psi_{0}\left(K_{2}, X_{2}\right)\right|^{2}\right) \\
& \times\left(\delta K-A P K^{\alpha}\left(1-\kappa \int \frac{P_{3} \exp \left(-\frac{\left|X-X_{2}\right|+\left|X_{2}-X_{3}\right|}{d}\right)}{P_{2}^{\gamma}}\left|\Psi_{0}\left(K_{2}, X_{2}\right)\right|^{2}\left|\Psi_{0}\left(K_{3}, X_{3}\right)\right|^{2}\right)\right) \Psi_{0}(K, X)
\end{aligned}
$$

As before, the first term is the potential in state $\Psi_{0}$ and can be discarded. Under our asumptions, the last term rewrites:

$$
\begin{aligned}
& +\int\left(\int G_{0}\left(X_{3}, X_{3}\right) G\left(X_{2}, X_{2}\right)\left(\langle K\rangle_{X}\right)^{\frac{\alpha \gamma}{1+\gamma}}\left(\langle K\rangle_{X, 0}\right)^{-\frac{\alpha}{1+\gamma}} f\left(X_{3}\right)\left(f\left(X_{2}\right)\right)^{-\gamma} \exp \left(-\frac{\left|X-X_{2}\right|+\left|X_{2}-X_{3}\right|}{d}\right)\right. \\
& \left.+\int G_{0}\left(X_{2}, X_{2}\right) G\left(X_{3}, X_{3}\right)\left(\langle K\rangle_{X, 0}\right)^{\frac{\alpha \gamma}{1+\gamma}}\left(\langle K\rangle_{X}\right)^{-\frac{\alpha}{1+\gamma}} \exp \left(-\frac{\left|X-X_{2}\right|+\left|X_{2}-X_{3}\right|}{d}\right)\right) \\
& \times\left(\delta\langle K\rangle_{X, 0}-A P\langle K\rangle_{X, 0}^{\alpha}\left(1-\kappa \int\langle K\rangle_{X_{2}, 0}^{\frac{\alpha \gamma}{1+\gamma}}\langle K\rangle_{X_{3}, 0}^{-\frac{\alpha}{1+\gamma}} f\left(X_{3}\right)\left(f\left(X_{2}\right)\right)^{-\gamma} \exp \left(-\frac{\left|X-X_{2}\right|+\left|X_{2}-X_{3}\right|}{d}\right)\right)\right) \\
& \times G_{0}(X, X)
\end{aligned}
$$

and is nul in first approximation since:

$$
0=\delta\langle K\rangle_{X, 0}-A P\langle K\rangle_{X, 0}^{\alpha}\left(1-\kappa \int\langle K\rangle_{X_{2}, 0}^{\frac{\alpha \gamma}{1+\gamma}}\langle K\rangle_{X_{3}, 0}^{-\frac{\alpha}{1+\gamma}} f\left(X_{3}\right)\left(f\left(X_{2}\right)\right)^{-\gamma} \exp \left(-\frac{\left|X-X_{2}\right|+\left|X_{2}-X_{3}\right|}{d}\right)\right)
$$

holds for all $X$. Thus, the second order expansion becomes:

$\delta \Psi^{\dagger}(K, X)\left(\delta K-A P K^{\alpha}\left(1-\kappa \int\langle K\rangle_{X_{2}, 0}^{\frac{\alpha \gamma}{1+\gamma}}\langle K\rangle_{X_{3}, 0}^{-\frac{\alpha}{1+\gamma}} f\left(X_{3}\right)\left(f\left(X_{2}\right)\right)^{-\gamma} \exp \left(-\frac{\left|X-X_{2}\right|+\left|X_{2}-X_{3}\right|}{d}\right)\right)\right)^{2} \delta \Psi(K, X)$

Along with the constraint, we deduce that $f(X) \simeq f_{0}(X)$ and $\langle K\rangle_{X} \simeq\langle K\rangle_{X, 0}$, the values computed in state $\Psi_{0}$ previously in this Appendix. Thus, the potential becomes:

$$
\begin{aligned}
& \delta \Psi^{\dagger}\left(\delta K-A P K^{\alpha}\left(1-\kappa \int\langle K\rangle_{X_{2}, 0}^{\frac{\alpha \gamma}{1+\gamma}}\langle K\rangle_{X_{3}, 0}^{-\frac{\alpha}{1+\gamma}} f\left(X_{3}\right)\left(f\left(X_{2}\right)\right)^{-\gamma} \exp \left(-\frac{\left|X-X_{2}\right|+\left|X_{2}-X_{3}\right|}{d}\right)\right)\right)^{2} \delta \Psi \\
= & \delta \Psi^{\dagger} \delta^{2}\left(K-\langle K\rangle_{X, 0}\right)^{2} \delta \Psi
\end{aligned}
$$

as a consequence of (87).

As in the case of the first phase, we can include the first order corrections in $\frac{A^{2}}{A^{2}}$. Since the terms appearing in the second order expansion are (86) and (88), the potential becomes:

$$
\delta^{2}\left(1+\frac{\bar{A}^{2} A^{2}}{\left(A^{2} U^{2}+\bar{A}^{2}\right)^{2}}\right) \delta \Psi^{\dagger}\left(\delta K-\langle K\rangle_{X, 0}\right)^{2} \delta \Psi
$$




\subsection{Appendix 4}

In this section, we compute the Green functions in both phases.

\subsubsection{Case $\rho=0$}

For $\rho=0$, without the $X$ contribution:

$$
\Psi^{\dagger}(K, P, X, \theta)\left(-\sigma^{2} \nabla_{K}^{2}-\vartheta^{2} \nabla_{\theta}^{2}+\left(\delta^{2}+\frac{\bar{A}^{2} A^{2}}{\left(A^{2} U^{2}+\bar{A}^{2}\right)^{2}}\right)(K-\langle K\rangle)^{2}+\frac{1}{\vartheta^{2}}+\alpha\right) \Psi(K, P, X, \theta)
$$

whose Green function is given by:

$$
\begin{aligned}
G_{0}\left(K, K^{\prime}, \theta, \theta^{\prime}, t\right)= & \sqrt{\frac{\omega}{2 \pi \sigma^{2} \sinh (\omega t)}} \exp \left(\left(-\frac{\omega}{2 \sigma^{2} \sinh (\omega t)}\right)\left(\left(K^{2}+\left(K^{\prime}\right)^{2}\right) \cosh (\omega t)-2 K K^{\prime}\right)\right) \\
& \times \sqrt{\frac{1}{2 \pi \vartheta^{2} t} \exp \left(-\frac{\left(\theta-\theta^{\prime}\right)^{2}}{\vartheta^{2} t}\right)}
\end{aligned}
$$

with:

$$
\omega=\delta^{2}+\frac{\bar{A}^{2} A^{2}}{\left(A^{2} U^{2}+\bar{A}^{2}\right)^{2}}
$$

To include the $\Psi(x)$ part of the action:

$$
\begin{aligned}
& \Psi^{\dagger}(x)\left(-\sigma_{X}^{2} \nabla_{X}^{2}+\kappa_{0} x^{2}\right) \Psi(x)-\frac{\kappa_{1}}{2}\left(\Psi^{\dagger}(K, x) \Psi(K, x)\right) \frac{K K^{\prime} \exp \left(-\chi_{1}|x-y|\right)}{\langle K\rangle_{x}\langle K\rangle_{y}}\left(\Psi^{\dagger}\left(K^{\prime}, y\right) \Psi\left(K^{\prime}, y\right)\right) \\
& +\frac{\kappa_{2}}{3}\left(\Psi^{\dagger}(K, x) \Psi(K, x)\right) \exp \left(-\chi_{2}|x-y|-\chi_{2}|x-z|-\chi_{2}|y-z|\right)\left(\Psi^{\dagger}\left(K^{\prime}, y\right) \Psi\left(K^{\prime}, y\right)\right)\left(\Psi^{\dagger}\left(K^{\prime \prime}, z\right) \Psi\left(K^{\prime \prime}, z\right)\right)
\end{aligned}
$$

and its contribution to the Green function, we can replace the interaction potential by its average in variables $y$ and $z$ :

$$
\begin{aligned}
& -\frac{\kappa_{1}}{2}|\Psi(K, x)|^{2} \frac{K K^{\prime} \exp \left(-\chi_{1}|x-y|\right)}{\langle K\rangle_{x}\langle K\rangle_{y}}\left|\Psi\left(K^{\prime}, y\right)\right|^{2} \\
\rightarrow & -\kappa_{1}\left\langle\frac{K K^{\prime} \exp \left(-\chi_{1}|x-y|\right)}{\langle K\rangle_{x}\langle K\rangle_{y}}\left|\Psi\left(K^{\prime}, y\right)\right|^{2}\right\rangle|\Psi(K, x)|^{2} \\
& \quad \frac{\kappa_{2}}{3}|\Psi(K, x)|^{2} \exp \left(-\chi_{2}|x-y|-\chi_{2}|x-z|-\chi_{2}|y-z|\right)\left|\Psi\left(K^{\prime}, y\right)\right|^{2}\left|\Psi\left(K^{\prime \prime}, z\right)\right|^{2} \\
\rightarrow & \kappa_{2}|\Psi(K, x)|^{2}\left\langle\exp \left(-\chi_{2}|x-y|-\chi_{2}|x-z|-\chi_{2}|y-z|\right)\left|\Psi\left(K^{\prime}, y\right)\right|^{2}\left|\Psi\left(K^{\prime \prime}, z\right)\right|^{2}\right\rangle
\end{aligned}
$$

Given that at the lowest order:

$$
\left\langle\frac{K K^{\prime} \exp \left(-\chi_{1}|x-y|\right)}{\langle K\rangle_{x}\langle K\rangle_{y}}\left|\Psi\left(K^{\prime}, y\right)\right|^{2}\right\rangle \simeq \int \frac{K K^{\prime} \exp \left(-\chi_{1}|x-y|\right)}{\langle K\rangle_{x}\langle K\rangle_{y}} G\left((K, x),\left(K^{\prime}, y\right)\right) G\left(\left(K^{\prime}, y\right),(K, x)\right) d y
$$

Under the same hypothesis as in appendix 2, that is $K^{\prime}$ is spread around $\langle K\rangle_{y}$ and the $X$ part of the Green function is approximatively uniformly distributed on the interval $[-1,1]$, we are left with:

$$
\left\langle\frac{K K^{\prime} \exp \left(-\chi_{1}|x-y|\right)}{\langle K\rangle_{x}\langle K\rangle_{y}}\left|\Psi\left(K^{\prime}, y\right)\right|^{2}\right\rangle \simeq \frac{K}{2\langle K\rangle_{x}} \int \exp \left(-\chi_{1}|x-y|\right) d y
$$


This last integral is:

$$
\begin{aligned}
& \int_{-1}^{1} \exp \left(-\left(\chi_{1}\right)|x-y|\right) d y \\
= & \int_{-1-x}^{1-x} \exp \left(-\chi_{1}|u|\right) d u=\int_{-1-x}^{0} \frac{\exp \left(\chi_{1} u\right)}{\sqrt{\alpha}} d u+\int_{0}^{1-x} \frac{\exp \left(-\chi_{1} u\right)}{\sqrt{\alpha}} d u \\
= & \frac{\left(2-\exp \left(-\chi_{1}(1+x)\right)-\exp \left(-\chi_{1}(1-x)\right)\right)}{\chi_{1}} \\
= & \frac{2\left(1-\exp \left(-\chi_{1}\right) \cosh \left(\chi_{1} x\right)\right)}{\chi_{1}} \\
= & \frac{2\left(1-\exp \left(-\chi_{1}\right)\right)}{\chi_{1}}+\frac{2\left(\exp \left(-\chi_{1}\right)\right)\left(1-\cosh \left(\chi_{1} x\right)\right)}{\chi_{1}} \\
\simeq & \frac{2\left(1-\exp \left(-\chi_{1}\right)\right)}{\chi_{1}}-\exp \left(-\chi_{1}\right) \chi_{1} x^{2}
\end{aligned}
$$

By the same token, we find the evaluation of the second part of the potential:

$$
\begin{aligned}
& \int \kappa_{2}\left\langle\exp \left(-\chi_{2}|x-y|-\chi_{2}|x-z|-\chi_{2}|y-z|\right)|\Psi(x)|^{2}|\Psi(y)|^{2}|\Psi(z)|^{2}\right\rangle \\
\rightarrow & \int \kappa_{2} \exp \left(-\chi_{2}|x-y|-\chi_{2}|x-z|-\chi_{2}|y-z|\right) G(x, y) G(y, z) G(z, x) d y d z \\
\simeq & \int \kappa_{2} \exp \left(-\chi_{2}(|x-y|+|x-z|+|y-z|)\right) d y d z \\
\simeq & \int \kappa_{2} \exp \left(-\chi_{2}(|x-y|+|y-x|)\right) d y \simeq \kappa_{2} \frac{2\left(1-\exp \left(-\chi_{2}\right)\right)}{\chi_{2}}-\kappa_{2} \exp \left(-\chi_{2}\right) \chi_{2} x^{2}
\end{aligned}
$$

Under these approximations, the $X$ part of the action becomes:

$$
(\nabla \Psi(x))^{2}+\omega_{X} \Psi^{\dagger}(x) x^{2} \Psi(x)+\alpha_{X} \Psi^{\dagger}(x) \Psi(x)
$$

where:

and:

$$
\omega_{X}=\kappa_{0}+\kappa_{1} \frac{K}{\langle K\rangle_{X}} \exp \left(-\chi_{1}\right) \chi_{1}-\kappa_{2} \exp \left(-\chi_{2}\right) \chi_{2}
$$

$$
\alpha_{X}=\alpha-\kappa_{1} \frac{K}{\langle K\rangle_{X}} \frac{2\left(1-\exp \left(-\chi_{1}\right)\right)}{\chi_{1}}+\kappa_{2} \frac{2\left(1-\exp \left(-\chi_{2}\right)\right)}{\chi_{2}}
$$

As a consequence, the overall second order action becomes:

$\Psi^{\dagger}(K, X, \theta)\left(-\sigma^{2} \nabla_{K}^{2}-\vartheta^{2} \nabla_{\theta}^{2}-\sigma_{X}^{2} \nabla_{X}^{2}+\left(\delta^{2}+\frac{\bar{A}^{2} A^{2}}{\left(A^{2} U^{2}+\bar{A}^{2}\right)^{2}}\right)(K-\langle K\rangle)^{2}+\omega_{X} x^{2}+\frac{1}{\vartheta^{2}}+\alpha_{X}\right) \Psi(K, X, \theta)$

Ultimately, the Green function are modified by the change of variable (59) including a factor:

$$
\exp \left(-\int\left(\frac{\left(\delta K-A P K^{\alpha}+V_{1}\right)}{\sigma^{2}}\right)\right) \Psi(K, P, X, \theta)
$$

with:

$$
V_{1}=\kappa A P K^{\alpha} \int \frac{P_{3} \exp \left(-\left(\left|X_{1}-X_{2}\right|+\left|X_{1}-X_{3}\right|\right)\right)}{P_{2}^{\gamma}}\left|\Psi\left(K_{2}, P_{2}, X_{2}, \theta\right)\right|^{2}\left|\Psi\left(K_{3}, P_{3}, X_{3}, \theta\right)\right|^{2}
$$

Given that:

$$
\begin{aligned}
& \int A P K^{\alpha}-V_{1} \\
\simeq & \int A P K^{\alpha}\left(1-\kappa\left\langle\int \frac{P_{3} \exp \left(-\left(\left|X_{1}-X_{2}\right|+\left|X_{1}-X_{3}\right|\right)\right)}{P_{2}^{\gamma}}\left|\Psi\left(K_{2}, P_{2}, X_{2}, \theta\right)\right|^{2}\left|\Psi\left(K_{3}, P_{3}, X_{3}, \theta\right)\right|^{2}\right\rangle\right) \\
= & \int \kappa A P K^{\alpha} U
\end{aligned}
$$


the exponential factor rewrites:

$$
\begin{aligned}
\exp \left(-\int\left(\frac{\left(\delta K-A P K^{\alpha}+V_{1}\right)}{\sigma^{2}}\right)\right) & =\exp \left(-\int\left(\frac{\left(\delta K-A P K^{\alpha} U\right)}{\sigma^{2}}\right)\right) \\
& =\exp \left(-\int\left(\frac{\left(\delta K-A(K)^{\frac{\alpha \gamma}{1+\gamma}} f(X) U\right)}{\sigma^{2}}\right)\right)
\end{aligned}
$$

We have seen in Appendix 2 that:

$$
\delta\langle K\rangle_{X}-A\left(\langle K\rangle_{X}\right)^{\frac{\alpha \gamma}{1+\gamma}} f(X) U=0
$$

so that we can rewrite the term in the exponential as:

$$
\begin{aligned}
\frac{\delta}{\sigma^{2}} \int\left(K-\left(\frac{K}{\langle K\rangle_{X}}\right)^{\frac{\alpha \gamma}{1+\gamma}}\langle K\rangle_{X}\right) & \simeq \frac{\delta}{\sigma^{2}} \int\left(K-\langle K\rangle_{X}\right) \\
& =\frac{\left(K-\langle K\rangle_{X}\right)^{2}}{2 \sigma^{2}}
\end{aligned}
$$

for $\gamma<<1$. The action (89) is now quadratic, but the variables $K$ and $X$ are entangled through $\omega$ and $\omega_{X}$. To find the Green function between $K, X$ and $K^{\prime}, X^{\prime}$ one can simplify the problem by replacing $K, X$ in $\omega$ and $\omega_{X}$ by their average trajectory values. In first approximation this means to replace $K, X$ with $\frac{\langle K\rangle_{X}+\langle K\rangle_{X^{\prime}}}{2}, \frac{X+X^{\prime}}{2}$. Then we set:

$$
\begin{gathered}
\langle K\rangle=\frac{\langle K\rangle_{X}+\langle K\rangle_{X^{\prime}}}{2} \\
\bar{\omega}_{X}=\kappa_{0}+\frac{\kappa_{1}}{2}\left(\frac{K}{\langle K\rangle_{X}}+\frac{K^{\prime}}{\langle K\rangle_{Y}}\right) \exp \left(-\chi_{1}\right) \chi_{1}-\kappa_{2} \exp \left(-\chi_{2}\right) \chi_{2} \\
\bar{\alpha}_{X}=\alpha+\frac{1}{\vartheta^{2}}-\frac{\kappa_{1}}{2}\left(\frac{K}{\langle K\rangle_{X}}+\frac{K^{\prime}}{\langle K\rangle_{Y}}\right) \frac{2\left(1-\exp \left(-\chi_{1}\right)\right)}{\chi_{1}}+\kappa_{2} \frac{2\left(1-\exp \left(-\chi_{2}\right)\right)}{\chi_{2}} \\
\bar{U}=\left(1-\frac{(\gamma+1)^{2}}{(\gamma+2)(2 \gamma+1)}\left(\frac{1+\gamma}{4}\left(\left(\frac{2}{1+\gamma}\right)^{2}-1\right)\right)^{\frac{1+\gamma}{1-\gamma}} \exp \left(-\left|\frac{X+X^{\prime}}{2}\right|\right)\right) \\
\bar{\omega}=\delta^{2}+\frac{\bar{A}^{2} A^{2}}{\left(A^{2} \bar{U}^{2}+\bar{A}^{2}\right)^{2}}
\end{gathered}
$$

and the Green function of the action (89) is the Laplace transform with parameter $\bar{\alpha}_{X} \simeq \alpha$ for small coupling parameters, of the following temporal transition function :

$$
\begin{aligned}
& G\left(K, K^{\prime}, P, P^{\prime}, X, X^{\prime}, \theta, \theta^{\prime}, t\right) \\
= & \exp \left(-\left[\frac{\left(K-\langle K\rangle_{X}\right)^{2}}{2 \sigma^{2}}\right]_{(K, X)}^{\left(K^{\prime}, X^{\prime}\right)}\right) \\
& \times \sqrt{\frac{\bar{\omega}}{2 \pi \sigma^{2} \sinh (\bar{\omega} t)}} \exp \left(-\frac{\bar{\omega}\left(\left((K-\langle K\rangle)^{2}+\left(K^{\prime}-\langle K\rangle\right)^{2}\right) \cosh (\bar{\omega} t)-2(K-\langle K\rangle)\left(K^{\prime}-\langle K\rangle\right)\right)}{2 \sigma^{2} \sinh (\bar{\omega} t)}\right) \\
& \times \sqrt{\frac{\bar{\omega}_{X}}{2 \pi \sinh \left(\bar{\omega}_{X} t\right)}} \exp \left(-\frac{\bar{\omega}_{X}\left(\left(X^{2}+\left(X^{\prime}\right)^{2}\right) \cosh \left(\bar{\omega}_{X} t\right)-2 X X^{\prime}\right)}{2 \sinh \left(\bar{\omega}_{X} t\right)}\right) \\
& \times \sqrt{\frac{1}{2 \pi \vartheta^{2} t}} \exp \left(-\frac{\left(\theta-\theta^{\prime}-t\right)^{2}}{2 \vartheta^{2} t}\right) \times \delta\left(P-\frac{D \exp \left(-\frac{|X|}{1+\gamma}\right)}{(K)^{\frac{\alpha}{1+\gamma}}}\right) \delta\left(P^{\prime}-\frac{D \exp \left(-\frac{\left|X^{\prime}\right|}{1+\gamma}\right)}{\left(K^{\prime}\right)^{\frac{\alpha}{1+\gamma}}}\right)
\end{aligned}
$$


For $\vartheta^{2}<<1$, The variable $t$ can be replaced by $\theta-\theta^{\prime}$, for $\theta>\theta^{\prime}$. Actually, due to the term:

$$
\exp \left(-\frac{\left(\theta-\theta^{\prime}-t\right)^{2}}{2 \vartheta^{2} t}\right)
$$

the Green function is non nul for values of $\theta$ and $\theta^{\prime}$ such that $\theta-\theta^{\prime}-t=0$. Since $t>0$, this implies that the replacement is only valid for $\theta>\theta^{\prime}$, otherwise the Green function is equal to 0 . As a consequence, we can remove the time dependence in the Green function and we obtain:

$$
\begin{aligned}
& G\left(K, K^{\prime}, P, P^{\prime}, X, X^{\prime}, \theta, \theta^{\prime}\right) \\
= & \exp \left(-\left[\frac{\left(K-\langle K\rangle_{X}\right)^{2}}{2 \sigma^{2}}\right]_{(K, X)}^{\left(K^{\prime}, X^{\prime}\right)}\right) \\
& \times \sqrt{\frac{\bar{\omega}}{2 \pi \sigma^{2} \sinh \left(\bar{\omega}\left(\theta-\theta^{\prime}\right)\right)}} \exp \left(-\frac{\bar{\omega}\left(\left((K-\langle K\rangle)^{2}+\left(K^{\prime}-\langle K\rangle\right)^{2}\right) \cosh \left(\bar{\omega}\left(\theta-\theta^{\prime}\right)\right)-2(K-\langle K\rangle)\left(K^{\prime}-\langle K\rangle\right)\right)}{2 \sigma^{2} \sinh \left(\bar{\omega}\left(\theta-\theta^{\prime}\right)\right)}\right) \\
& \times \sqrt{\frac{\bar{\omega}_{X}}{2 \pi \sinh \left(\bar{\omega}_{X}\left(\theta-\theta^{\prime}\right)\right)}} \exp \left(-\frac{\bar{\omega}_{X}\left(\left(X^{2}+\left(X^{\prime}\right)^{2}\right) \cosh \left(\bar{\omega}_{X}\left(\theta-\theta^{\prime}\right)\right)-2 X X^{\prime}\right)}{2 \sinh \left(\bar{\omega}_{X}\left(\theta-\theta^{\prime}\right)\right)}\right) \\
& \times \delta\left(P-\frac{D \exp \left(-\frac{|X|}{1+\gamma}\right)}{(K)^{\frac{\alpha}{1+\gamma}}}\right) \delta\left(P^{\prime}-\frac{D \exp \left(-\frac{\left|X^{\prime}\right|}{1+\gamma}\right)}{\left(K^{\prime}\right)^{\frac{\alpha}{1+\gamma}}}\right) H\left(\theta-\theta^{\prime}\right)
\end{aligned}
$$

where $H\left(\theta-\theta^{\prime}\right)$ is the Heaviside function.

Note that for $\theta-\theta^{\prime}=0$, in (90), the dominant part becomes $\frac{t}{2 \vartheta^{2}}$, which means that in average one can replace $t$ by $\vartheta^{2}$, which describes the description in terms of harmonic oscillators used in Appendix 2 .

\subsubsection{Case $\rho \neq 0$}

For $\rho \neq 0$, the $X$ part of the action is similar to the previous case, in

$$
\begin{aligned}
& \int \Psi^{\dagger}(K, X)\left(-\nabla_{X}^{2}+\kappa_{0} X^{2}\right) \Psi(K, X)-\frac{\kappa_{1}}{2}|\Psi(K, X)|^{2} \frac{K K^{\prime} \exp \left(-\chi_{1}|X-Y|\right)}{\langle K\rangle^{2}}\left|\Psi\left(K^{\prime}, Y\right)\right|^{2} \\
& +\int \frac{\kappa_{2}}{3} \exp \left(-\chi_{2}|X-Y|-\chi_{2}|X-Z|-\chi_{2}|Y-Z|\right)|\Psi(K, X)|^{2}\left|\Psi\left(K^{\prime}, Y\right)\right|^{2}\left|\Psi\left(K^{\prime \prime}, Z\right)\right|^{2}
\end{aligned}
$$

we will replace the interaction potential by its average in variables $y$ and $z$, but now, this average is computed in the fundamental state $\Psi_{0}$. We found that the norm of $\Psi_{0}$ satisfies at the zeroth order in $\chi_{1}$ and $\chi_{2}$ :

$$
\rho^{2}=\frac{\kappa_{1}+\sqrt{\kappa_{1}^{2}-2 \kappa_{2}\left(2 \alpha+\frac{2}{\vartheta^{2}}+\sqrt{\kappa_{0}}+\sigma \delta\right)}}{2 \kappa_{2}}
$$

for $\bar{A}^{2}>>A^{2}$. It will be useful in the sequel to find a more precise form for $\rho^{2}$ at the second order in $\chi_{1}$ and $\chi_{2}$. At this order, the equation for the fundamental $\Psi_{0}$ can be found in the following way. We developp 
the potential terms to second order in $\Psi(K, x)$ around $\Psi_{0}$, that is:

$$
\begin{aligned}
& \int \frac{\kappa_{1}}{2}|\Psi(K, X)|^{2} \frac{K K^{\prime} \exp \left(-\chi_{1}|X-Y|\right)}{\langle K\rangle^{2}}\left|\Psi\left(K^{\prime}, Y\right)\right|^{2} d Y \\
\rightarrow & \int \kappa_{1}|\Psi(K, X)|^{2} \frac{K K^{\prime} \exp \left(-\chi_{1}|X-Y|\right)}{\langle K\rangle^{2}}\left|\Psi_{0}\left(K^{\prime}, Y\right)\right|^{2} d Y \\
\simeq & \kappa_{1} \exp \left(-\chi_{1}|X|\right)|\Psi(K, X)|^{2} \frac{K}{\langle K\rangle} \rho^{2} \\
\simeq & \kappa_{1}\left(\frac{K}{\langle K\rangle} \exp \left(-\chi_{1}|X|\right)|\Psi(K, X)|^{2}\right) \rho^{2}
\end{aligned}
$$

The parameter $\rho^{2}$ has been computed before. Similarly, the second part of the potential is replaced by:

$$
\begin{aligned}
& \frac{\kappa_{2}}{3}\left|\Psi_{0}(K, x)\right|^{2} \exp \left(-\chi_{2}|x-y|-\chi_{2}|x-z|-\chi_{2}|y-z|\right)\left|\Psi\left(K^{\prime}, y\right)\right|^{2}\left|\Psi^{\dagger}\left(K^{\prime \prime}, z\right)\right|^{2} \\
& \rightarrow \int \kappa_{2} \exp \left(-\chi_{2}|x-y|-\chi_{2}|x-z|-\chi_{2}|y-z|\right)\left|\Psi_{0}(K, x)\right|^{2}\left|\Psi_{0}\left(K^{\prime}, y\right)\right|^{2}\left|\Psi_{0}\left(K^{\prime \prime}, z\right)\right|^{2} d K^{\prime} d K^{\prime \prime} d y d z \\
& \simeq \kappa_{2} \exp \left(-2 \chi_{2}|x|\right)\left|\Psi_{0}(K, x)\right|^{2} \rho^{4} \\
&-\nabla^{2} \Psi(x)+\frac{\kappa_{0}}{2} x^{2} \Psi(x)-\kappa_{1}\left(\frac{K}{\langle K\rangle} \exp \left(-\chi_{1}|x|\right)|\Psi(K, x)|^{2}\right) \rho^{2}+\kappa_{2} \exp \left(-2 \chi_{2}|x|\right)|\Psi(K, x)|^{2} \rho^{4}+\alpha \Psi(x) \\
& \text { expanding the exponentials in } \chi_{1} \text { and } \chi_{2} \text { yields thus the first approximation to the potential of the } X \\
&\left(\text { (79), } \text { and thus a second order action in } \chi_{1} \text { and } \chi_{2}:\right. \\
& \quad \Psi^{\dagger}(x)\left(-\sigma_{X}^{2} \nabla_{X}^{2}+\frac{\kappa_{0}}{2}\left(1-\frac{\chi_{1}^{2} \kappa_{1} \rho^{2}}{2 \kappa_{0}}+2 \chi_{2} \frac{\kappa_{2}}{\kappa_{0}} \rho^{4}\right)\left(x+\frac{\operatorname{sgn}(x)}{\kappa_{0}}\left(\chi_{1} \kappa_{1} \frac{K}{2\langle K\rangle} \rho^{2}-\chi_{2} \kappa_{2} \rho^{4}\right)\right)^{2}\right) \Psi(x) \\
&+\left(\rho^{4} \kappa_{2}-\rho^{2} \kappa_{1}-\frac{1}{2 \kappa_{0}}\left(\chi_{1} \kappa_{1} \frac{K}{2\langle K\rangle} \rho^{2}-\chi_{2} \kappa_{2} \rho^{4}\right){ }^{2}\right)\left(\Psi^{\dagger}(x) \Psi(x)\right)
\end{aligned}
$$

The complete action is then:

$$
\begin{aligned}
& \int \Psi^{\dagger}(K, X, \theta)\left(-\sigma^{2} \nabla_{K}^{2}-\vartheta^{2} \nabla_{\theta}^{2}-\sigma_{X}^{2} \nabla_{X}^{2}+\left(\delta^{2}+\frac{\bar{A}^{2} A^{2}}{\left(A^{2} U^{2}+\bar{A}^{2}\right)^{2}}\right)(K-\langle K\rangle)^{2}\right) \Psi(K, X, \theta) \\
& +\frac{\kappa_{0}}{2}\left(1-\frac{\chi_{1}^{2} \kappa_{1} \rho^{2}}{2 \kappa_{0}}+2 \chi_{2} \frac{\kappa_{2}}{\kappa_{0}} \rho^{4}\right) \int\left(\left(x+\frac{\operatorname{sgn}(x)}{\kappa_{0}}\left(\chi_{1} \kappa_{1} \frac{K}{2\langle K\rangle} \rho^{2}-\chi_{2} \kappa_{2} \rho^{4}\right)\right)^{2} x^{2}+\alpha_{X}\right)|\Psi(K, X, \theta)|^{2} \\
& +\left(2 \rho^{2} \kappa_{2}-\kappa_{1}\right)\left|\int \Psi^{\dagger}(K, X, \theta) \Psi_{0}(K, X, \theta)\right|^{2}
\end{aligned}
$$

where

$$
\alpha_{X}=\alpha+\frac{1}{\vartheta^{2}}+\left(-\kappa_{1} \rho^{2}+\kappa_{2} \rho^{4}-\frac{1}{2 \kappa_{0}}\left(\chi_{1} \kappa_{1} \frac{K}{2\langle K\rangle} \rho^{2}-\chi_{2} \kappa_{2} \rho^{4}\right)^{2}\right)
$$

since (85) implies that:

$$
\begin{aligned}
0= & \alpha+\frac{1}{\vartheta^{2}}+\frac{1}{2} \kappa_{0}^{\frac{1}{2}} \sqrt{1-\frac{\chi_{1}^{2} \kappa_{1} \rho^{2}}{2}+2 \chi_{2}^{2} \kappa_{2} \rho^{4}} \\
& -\kappa_{1} \rho^{2}+\kappa_{2} \rho^{4}-\frac{1}{2 \kappa_{0}}\left(\chi_{1} \kappa_{1} \frac{K}{2\langle K\rangle} \rho^{2}-\chi_{2} \kappa_{2} \rho^{4}\right)^{2}+\frac{\sigma \sqrt{\delta^{2}+\frac{\bar{A}^{2} A^{2}}{\left(A^{2} U^{2}+\bar{A}^{2}\right)^{2}}}}{2}
\end{aligned}
$$


one has:

$$
\alpha_{X}=-\frac{1}{2} \kappa_{0}^{\frac{1}{2}} \sqrt{1-\frac{\chi_{1}^{2} \kappa_{1} \rho^{2}}{2}+2 \chi_{2}^{2} \kappa_{2} \rho^{4}}-\frac{\sigma \sqrt{\delta^{2}+\frac{\bar{A}^{2} A^{2}}{\left(A^{2} U^{2}+\bar{A}^{2}\right)^{2}}}}{2}
$$

and the second order action rewrites:

$$
\begin{aligned}
& \int \Psi^{\dagger}(K, X, \theta)\left(-\sigma^{2} \nabla_{K}^{2}-\vartheta^{2} \nabla_{\theta}^{2}-\sigma_{X}^{2} \nabla_{X}^{2}+\left(\delta^{2}+\frac{\bar{A}^{2} A^{2}}{\left(A^{2} U^{2}+\bar{A}^{2}\right)^{2}}\right)(K-\langle K\rangle)^{2}\right) \Psi(K, X, \theta) \\
& +\frac{\kappa_{0}}{2}\left(1-\frac{\chi_{1}^{2} \kappa_{1} \rho^{2}}{2 \kappa_{0}}+2 \chi_{2} \frac{\kappa_{2}}{\kappa_{0}} \rho^{4}\right) \int \Psi^{\dagger}(K, X, \theta)\left(\left(x+\frac{\operatorname{sgn}(x)}{\kappa_{0}}\left(\chi_{1} \kappa_{1} \frac{K}{2\langle K\rangle} \rho^{2}-\chi_{2} \kappa_{2} \rho^{4}\right)\right)^{2} x^{2}\right) \Psi(K, X, \theta) \\
& -\int \Psi^{\dagger}(K, X, \theta)\left(\frac{1}{2} \kappa_{0}^{\frac{1}{2}} \sqrt{1-\frac{\chi_{1}^{2} \kappa_{1} \rho^{2}}{2}+2 \chi_{2}^{2} \kappa_{2} \rho^{4}}+\frac{\sigma \sqrt{\delta^{2}+\frac{\bar{A}^{2} A^{2}}{\left(A^{2} U^{2}+\bar{A}^{2}\right)^{2}}}}{2}\right) \Psi(K, X, \theta) \\
& +\left(2 \rho^{2} \kappa_{2}-\kappa_{1}\right)\left|\int \Psi^{\dagger}(K, P, X, \theta) \Psi_{0}(K, P, X, \theta)\right|^{2}
\end{aligned}
$$

As said before, the fundamental level of the $X$ part of the action has the form:

$$
\begin{aligned}
\Psi_{0}(X) & =N_{1}\left(\exp \left(-\frac{\kappa_{0}^{-\frac{1}{2}}(X+\delta X)^{2}}{2}\right) H(x)+\exp \left(-\frac{\kappa_{0}^{-\frac{1}{2}}(X-\delta X)^{2}}{2}\right) H(-x)\right) \\
& \equiv \bar{\Psi}_{0}(X+\delta X) H(X)+\bar{\Psi}_{0}(X-\delta X) H(-X)
\end{aligned}
$$

where:

$$
\delta X=\frac{1}{\kappa_{0}}\left(\chi_{1} \kappa_{1} \frac{K}{2\langle K\rangle} \rho^{2}-\chi_{2} \kappa_{2} \rho^{4}\right)
$$

For a given value of $K$, The Green function can be obtained by its expansion in function of all the eigenstates $\bar{\Psi}_{n}$ of the system.

$$
\begin{aligned}
G_{K}(x, y)= & \sum_{n}\left(\bar{\Psi}_{n}(x+\delta X) H(x)+\bar{\Psi}_{n}(x-\delta X) H(-x)\right) \times\left(\bar{\Psi}_{n}(y+\delta X) H(y)+\bar{\Psi}_{n}(y-\delta X) H(-y)\right) \\
= & \bar{G}(x+\delta X, y+\delta X) H(x) H(y)+\bar{G}(x-\delta X, y-\delta X) H(-x) H(-y) \\
& +\bar{G}(x+\delta X, y-\delta X) H(x) H(-y)+\bar{G}(x-\delta X, y+\delta X) H(-x) H(y)
\end{aligned}
$$

where we have defined:

$$
\begin{aligned}
\bar{G}(x, y, t) & =\sqrt{\frac{\kappa_{0}}{2 \pi \sigma_{X}^{2} \sinh \left(\kappa_{0} t\right)}} \exp \left(\left(-\frac{\kappa_{0}}{2 \sigma_{X}^{2} \sinh \left(\kappa_{0} t\right)}\right)\left(\left(x^{2}+\left(x^{\prime}\right)^{2}\right) \cosh \left(\kappa_{0} t\right)-2 x x^{\prime}\right)\right) \\
& =\sqrt{\frac{\kappa_{0}}{2 \pi \sigma_{X}^{2} \sinh \left(\kappa_{0} t\right)}} \exp \left(\left(-\frac{\kappa_{0}}{2 \sigma_{X}^{2} \sinh \left(\kappa_{0} t\right)}\right)\left(\left(x-x^{\prime}\right)^{2}+\left(\cosh \left(\kappa_{0} t\right)-1\right)\left(x^{2}+\left(x^{\prime}\right)^{2}\right)\right)\right)
\end{aligned}
$$

The term associated to the change of variable can be found similarly to the first phase:, but now it has to evaluated in the state $\Psi_{0}(K, X, \theta)+\Psi(K, X, \theta)$. As explained in Appendix 3, in first approximation, this is equivalent to evaluate it in the state $\Psi_{0}(K, X, \theta)$. We find again an exponential factor:

$$
\exp \left(-\left[{\frac{\left(K-\langle K\rangle_{X}\right)^{2}}{2 \sigma^{2}}}_{(K, X)}^{\left(K^{\prime}, X^{\prime}\right)}\right]_{(K, X)}^{\left(K^{\prime}, X^{\prime}\right)}\right)
$$

with $\langle K\rangle_{X}$ computed in the state $\Psi_{0}(K, X, \theta)$ as in Appendix 3. 
Ultimately, as in the first phase, to (92) one can associate a Green function that is the Laplace transform with parameter $\alpha_{X}$ of a temporal Green function:

$$
\begin{aligned}
& G\left(K, K^{\prime}, P, P^{\prime}, X, X^{\prime}, \theta, \theta^{\prime}, t\right) \\
= & \exp \left(-\left[\frac{\left(K-\langle K\rangle_{X}\right)^{2}}{2 \sigma^{2}}(K, X)\right]_{(K, X)}\right) \\
& \times \sqrt{\frac{\bar{\omega}}{2 \pi \sigma^{2} \sinh (\bar{\omega} t)}} \exp \left(-\frac{\bar{\omega}\left(\left((K-\langle K\rangle)^{2}+\left(K^{\prime}-\langle K\rangle\right)^{2}\right) \cosh (\bar{\omega} t)-2(K-\langle K\rangle)\left(K^{\prime}-\langle K\rangle\right)\right)}{2 \sigma^{2} \sinh (\bar{\omega} t)}\right) \\
& \times G_{\frac{K+K^{\prime}}{2}}(X, Y, t) \\
& \times \sqrt{\frac{1}{2 \pi \vartheta^{2} t}} \exp \left(-\frac{\left(\theta-\theta^{\prime}\right)^{2}}{2 \vartheta^{2} t}+\frac{\theta-\theta^{\prime}}{\vartheta^{2}}\right) \times \delta\left(P-\frac{D \exp \left(-\frac{|X|}{1+\gamma}\right)}{\left(\frac{K}{\langle K\rangle}\right)^{\frac{\alpha}{1+\gamma}}}\right) \times \delta\left(P^{\prime}-\frac{D \exp \left(-\frac{\left|X^{\prime}\right|}{1+\gamma}\right)}{\left(\frac{K^{\prime}}{\langle K\rangle}\right)^{\frac{\alpha}{1+\gamma}}}\right)
\end{aligned}
$$

Eventhough $\alpha_{X}<0$, the Laplace transform is well defined since $-\alpha_{X}$ is the lower bound of the terms in the exponential $\left(-\alpha_{X}\right.$ is the lowest eigenvalue associated to the evolution operator whose $G$ is the Green function).

As in phase 1 , this Green function is centered around $t=\theta-\theta^{\prime}$, so that we can replace $t=\theta-\theta^{\prime}$ in the Green function, leads to:

$$
\begin{aligned}
& G\left(K, K^{\prime}, P, P^{\prime}, X, X^{\prime}, \theta, \theta^{\prime}\right) \\
& =\exp \left(-\left[\frac{\left(K-\langle K\rangle_{X}\right)^{2}}{2 \sigma^{2}}{ }_{(K, X)}^{\left(K^{\prime}, X^{\prime}\right)}\right]_{(K, X)}^{\left(K^{\prime}, X^{\prime}\right)}\right) \\
& \times \sqrt{\frac{\bar{\omega}}{2 \pi \sigma^{2} \sinh \left(\bar{\omega}\left(\theta-\theta^{\prime}\right)\right)}} \exp \left(-\frac{\bar{\omega}\left(\left((K-\langle K\rangle)^{2}+\left(K^{\prime}-\langle K\rangle\right)^{2}\right) \cosh \left(\bar{\omega}\left(\theta-\theta^{\prime}\right)\right)-2(K-\langle K\rangle)\left(K^{\prime}-\langle K\rangle\right)\right)}{2 \sigma^{2} \sinh \left(\bar{\omega}\left(\theta-\theta^{\prime}\right)\right)}\right) \\
& \times G_{\frac{K+K^{\prime}}{2}}(X, Y) \times \delta\left(P-\frac{D \exp \left(-\frac{|X|}{1+\gamma}\right)}{\left(\frac{K}{\langle K\rangle}\right)^{\frac{\alpha}{1+\gamma}}}\right) \times \delta\left(P^{\prime}-\frac{D \exp \left(-\frac{\left|X^{\prime}\right|}{1+\gamma}\right)}{\left(\frac{K^{\prime}}{\langle K\rangle}\right)^{\frac{\alpha}{1+\gamma}}}\right) H\left(\theta-\theta^{\prime}\right)
\end{aligned}
$$

\subsection{Appendix 5}

We consider a general model:

$$
S(\Psi)=\int \Psi^{\dagger}(X)\left(-\sigma_{X}^{2} \nabla_{X}^{2}+V\right) \Psi(X)+\int \frac{1}{2} \Psi^{\dagger}(X) \Psi^{\dagger}(Y) W(X, Y) \Psi(X) \Psi(Y)
$$

that encompasses the model studied in this work. The field $\Psi(X)$ depends on an arbitrary number of variables $X$ belonging to some configuration space, and $W(X, Y)=W(Y, X)$. We have chosen a fourth order interaction term, but a more general choice, such a s a sum of powers, would not change the result. We assume that there is a non trivial minimum to the action $S(\Psi)$, so that the equation:

$$
\left(-\sigma_{X}^{2} \nabla_{X}^{2}+V\right) \Psi(X)+\left(\int \Psi^{\dagger}(Y) W(X, Y) \Psi(Y)\right) \Psi(X)=0
$$


has a solution $\rho \Psi_{0}(X) \neq 0$, and $\Psi_{0}$ of norm equal to 1 . We want to show that the non trivial vacuum implies to separate the system into two systems defined on two half-space of the configuration space.

Given the field $\Psi_{0}$ minimizing the action, the second order variation of $S(\Psi)$ is:

$$
\begin{aligned}
& \delta \Psi^{\dagger}(X)\left(-\sigma_{X}^{2} \nabla_{X}^{2}+V\right) \delta \Psi(X)+\delta \Psi^{\dagger}(X)\left(\int \Psi_{0}^{\dagger}(Y) W(X, Y) \Psi_{0}(Y)\right) \delta \Psi(X) \\
& +2 \operatorname{Re}\left(\delta \Psi^{\dagger}(X)\left(\Psi_{0}^{\dagger}(Y) W(X, Y) \Psi_{0}(X)\right) \delta \Psi(Y)\right) \\
= & \delta \Psi^{\dagger}(X)\left(-\sigma_{X}^{2} \nabla_{X}^{2}+V(X)\right) \delta \Psi(X)+\delta \Psi^{\dagger}(X) V_{0}(X) \delta \Psi(X)+\delta \Psi^{\dagger}(X) W_{0}(X, Y) \delta \Psi(Y)
\end{aligned}
$$

where:

$$
\begin{aligned}
W_{0}(Y, X) & =\Psi_{0}^{\dagger}(Y) W(X, Y) \Psi_{0}(X) \\
W_{0}^{\dagger}(X, Y) & =W_{0}(Y, X)
\end{aligned}
$$

and:

$$
V_{0}(X)=\int \Psi_{0}^{\dagger}(Y) W(X, Y) \Psi_{0}(Y)
$$

Now, assume that $\Psi_{0}(X)$ is peaked around some $X_{0}$, which is the case in the text for the $K$ part of $\Psi_{0}$, then:

$$
\begin{aligned}
V_{0}(X) & \simeq \int \Psi_{0}^{\dagger}(Y) W\left(X, X_{0}\right) \Psi_{0}(Y)=W\left(X, X_{0}\right) \rho^{2} \\
W_{0}(Y, X) & \simeq \rho^{2} \int \Psi_{0}^{\dagger}\left(X_{0}\right) W\left(X_{0}, X_{0}\right) \Psi_{0}(X) \delta\left(X-X_{0}\right) \delta\left(Y-X_{0}\right)
\end{aligned}
$$

and the following contributions of the second order variation becomes:

$$
\begin{aligned}
\delta \Psi^{\dagger}(X) V_{0}(X) \delta \Psi(X) & \rightarrow \rho^{2} \delta \Psi^{\dagger}(X) W\left(X, X_{0}\right) \delta \Psi(X) \\
\delta \Psi^{\dagger}(X) W_{0}(X, Y) \delta \Psi(Y) & \rightarrow \rho^{2}\left|\Psi_{0}(X)\right|^{2} W\left(X_{0}, X_{0}\right) \delta \Psi^{\dagger}\left(X_{0}\right) \delta \Psi\left(X_{0}\right)
\end{aligned}
$$

For $\rho^{2} W\left(X_{0}, X_{0}\right)>>1$, the contributions for fields $\delta \Psi$ such that $\delta \Psi\left(X_{0}\right) \neq 0$ are suppressed. Then the integrals over the $\delta \Psi$ can be limited to contributions such that $\delta \Psi\left(X_{0}\right)=0$.

This means that $\delta \Psi$ can be decomposed in two parts:

$$
\delta \Psi(X)=\delta \Psi_{+}(X)+\delta \Psi_{-}(X)
$$

and $\delta \Psi_{+}(X)$ and $\delta \Psi_{-}(X)$ are independent and defined on two half space $X_{ \pm}$respectively and satisfy:

$$
\delta \Psi_{ \pm}\left(X_{\mp}\right)=0
$$

And the second order action for $\delta \Psi_{ \pm}$becomes:

$$
\begin{aligned}
& \delta \Psi^{\dagger}(X)\left(-\sigma_{X}^{2} \nabla_{X}^{2}+V(X)\right) \delta \Psi(X)+\delta \Psi^{\dagger}(X) V_{0}(X) \delta \Psi(X)+\delta \Psi^{\dagger}(X) W_{0}(X, Y) \delta \Psi(Y) \\
= & \delta \Psi_{ \pm}^{\dagger}(X)\left(-\sigma_{X}^{2} \nabla_{X}^{2}+V(X)\right) \delta \Psi_{ \pm}(X)+\rho^{2} \delta \Psi_{ \pm}^{\dagger}(X) W\left(X, X_{0}\right) \delta \Psi_{ \pm}(X)
\end{aligned}
$$

This action models also two independent fields with constraint $\delta \bar{\Psi}_{ \pm}\left(X_{\mp}\right)=0$ and defined on the all space, but subject to a wall potential $H_{ \pm}(X)$, the wall being defined on the space $X_{\mp}$ :

$$
\delta \bar{\Psi}_{ \pm}^{\dagger}(X)\left(-\sigma_{X}^{2} \nabla_{X}^{2}+V(X)+H_{ \pm}(X)\right) \delta \bar{\Psi}_{ \pm}(X)+\rho^{2} \delta \bar{\Psi}_{ \pm}^{\dagger}(X) W\left(X, X_{0}\right) \delta \bar{\Psi}_{ \pm}(X)
$$

and this models two sets of different agents, evolving on $X_{ \pm}$and subject to a "wall" potential. Applied to our case, this result are the following. Recall that we found the fundamental state (82):

$$
\begin{aligned}
\Psi_{0}(K, X)= & \rho N\left[\bar{\Psi}_{0}(X+\delta X) H(X)+\bar{\Psi}_{0}(X-\chi \delta X) H(-X)\right] \\
& \times \exp \left(-\frac{\sqrt{\delta^{2}+\frac{\bar{A}^{2} A^{2}}{\left(A^{2} U^{2}+\bar{A}^{2}\right)^{2}}}\left(K-\langle K\rangle_{X}\right)^{2}}{2 \sigma}\right)
\end{aligned}
$$


where $N$ and $\rho$ are given by (83) and (176) and (84) respectively. If we assume, as in Appendix 2 that:

$$
\frac{\sqrt{\delta^{2}+\frac{\bar{A}^{2} A^{2}}{\left(A^{2} U^{2}+\bar{A}^{2}\right)^{2}}}}{\sigma}>\kappa_{0}^{\frac{1}{2}}
$$

i.e. the $X$ variable is more spread than $K$, then $\Psi_{0}(K, X)$ is peaked on the hypersurface $K=\langle K\rangle_{X}$. As a consequence, the space $(K, X)$ is divided into two subspaces $S_{+}$(defined by $\left.K>\langle K\rangle_{X}\right)$ and $S_{-}$(defined by $K<\langle K\rangle_{X}$ ). To these halfspaces correspond two systems, independent in first approximation. An agent starting in $S_{+}\left(S_{-}\right.$respectively) will remain in $S_{+}\left(S_{-}\right.$respectively). 


\section{References}

Abergel F, Chakraborti A, Muni Toke I and Patriarca M (2011) Econophysics review: I. Empirical facts, Quantitative Finance, Vol. 11, No. 7, 991-1012.

Abergel F, Chakraborti A, Muni Toke I and Patriarca M (2011) Econophysics review: II. Agent-based models, Quantitative Finance, Vol. 11, No. 7, 1013-1041.

Barro RJ, Sala-i-Martin X (1995) Economic Growth. McGraw-Hill, New-York.

Ciarli T, Lorentz A, Savona M, Valente M (2010) The effect of consumption and production structure on growth and distribution. A micro to macro model. Metroeconomica, 61(1):180.

Dawid H, Gemkow S, Harting P, van der Hoog S, Neugart M (2011) An agent-based macroeconomic model for economic policy analysis. Technical report, Working paper. Universitat Bielefeld.

Dosi G, Nelson RR (2010) Technical change and industrial dynamics as evolutionary processes. Handbook of the Economics of Innovation - Vol-I, p. 51-128.

Dosi G, Fagiolo G, Roventini A. Schumpeter meeting Keynes, A policy-friendly model of endogenous growth and business cycles. Journal of Economic Dynamics and Control, 34(9):1748 1767, September 2010.

Dosi G, Fagiolo G, Napoletano M, Roventini A, Treibich T (2015) Fiscal and monetary policies in complex evolving economies. Journal of Economic Dynamics and Control, 52:166.

Fujita M, Thisse J-F, Economics of Agglomeration, Cambridge, Cambridge University Press 2002.

Gaffard J-L and Napoletano M Editors: Agent-based models and economic policy. Ofce 2012.

Gualdi S, Mandel A (2016) Endogenous Growth in Production Networks. Documents de travail du Centre d'Economie de la Sorbonne 2016.54 - ISSN : 1955-611X.

Gosselin P, Lotz A and Wambst M (2017) A Path Integral Approach to Interacting Economic Systems with Multiple Heterogeneous Agents. IF_PREPUB. 2017. hal-01549586v2.

Gosselin P, Lotz A and Wambst M (2018) A Path Integral Approach to Business Cycle Models with Large Number of Agents arXiv:1810.07178 [econ.GN].

Handbook of Economic Growth, Volume 1, Part A, Pages 1-1060, I1-I46 (2005). Edited by Philippe Aghion and Steven N. Durlauf.

Jackson M (2010) Social and Economic Networks, Princeton University Press 2010.

Kleinert H (1989) Gauge fields in condensed matter Vol. I , Superflow and vortex lines, Disorder Fields, Phase Transitions, Vol. II, Stresses and defects, Differential Geometry, Crystal Melting, World Scientific, Singapore 1989.

Kleinert H (2009) Path Integrals in Quantum Mechanics, Statistics, Polymer Physics, and Financial Markets 5th edition, World Scientific, Singapore 2009.

Krugman P (1991) Increasing Returns and Economic Geography. Journal of Political Economy, 99(3), 483-499.

Lucas, Robert (1976) "Econometric Policy Evaluation: A Critique". In Brunner, K.; Meltzer, A. The Phillips Curve and Labor Markets. Carnegie-Rochester Conference Series on Public Policy. 1. New York: American Elsevier. pp. 19-46. ISBN 0-444-11007-0.

Mandel A, Jaeger C, Fürst S, Lass W, Lincke D, Meissner F, Pablo-Marti F, Wolf S. Agent-based dynamics in disaggregated growth models. Documents de travail du Centre d'Economie de la Sorbonne 10077, Université Pantheon-Sorbonne (Paris 1), Centre d'Economie de la Sorbonne, September 2010. 
Mandel A (2012) Agent-based dynamics in the general equilibrium model. Complexity Economics 1, 105121.

Solow RM (1957) Technical Change and the Aggregate Production Function. The Review of Economics and Statistics, Vol. 39, No. 3, pp. 312-320, August.

Wolf S, Fürst S, Mandel A, Lass W, Lincke D, Pablo-Marti F, Jaeger C (2013) A multi-agent model of several economic regions. Environmental Modelling and Software, Elsevier 44, pp.25-43. 